\title{
SDSS-IV MaNGA: the impact of diffuse ionized gas on emission-line ratios, interpretation of diagnostic diagrams and gas metallicity measurements
}

\author{
Kai Zhang, ${ }^{1 \star}$ Renbin Yan, ${ }^{1}$ Kevin Bundy, ${ }^{2}$ Matthew Bershady, ${ }^{3}$ \\ L. Matthew Haffner, ${ }^{3}$ René Walterbos, ${ }^{4}$ Roberto Maiolino, ${ }^{5,6}$ Christy Tremonti, ${ }^{3}$ \\ Daniel Thomas, ${ }^{7}$ Niv Drory, ${ }^{8}$ Amy Jones, ${ }^{9}$ Francesco Belfiore,, 6 \\ Sebastian F. Sánchez, ${ }^{10}$ Aleksandar M. Diamond-Stanic, ${ }^{3}$ Dmitry Bizyaev, ${ }^{11,12}$ \\ Christian Nitschelm, ${ }^{13}$ Brett Andrews, ${ }^{14}$ Jon Brinkmann, ${ }^{11}$ Joel R. Brownstein, ${ }^{15}$ \\ Edmond Cheung, ${ }^{2}$ Cheng Li, ${ }^{16,17}$ David R. Law, ${ }^{18}$ Alexandre Roman Lopes, ${ }^{19}$ \\ Daniel Oravetz, ${ }^{11}$ Kaike Pan, ${ }^{11}$ Thaisa Storchi Bergmann ${ }^{20,21}$ and Audrey Simmons ${ }^{11}$ \\ Affiliations are listed at the end of the paper
}

Accepted 2016 December 15. Received 2016 December 15; in original form 2016 June 1

\begin{abstract}
Diffuse ionized gas (DIG) is prevalent in star-forming galaxies. Using a sample of 365 nearly face-on star-forming galaxies observed by Mapping Nearby Galaxies at APO, we demonstrate how DIG in star-forming galaxies impacts the measurements of emission-line ratios, hence the interpretation of diagnostic diagrams and gas-phase metallicity measurements. At fixed metallicity, DIG-dominated low $\Sigma_{\mathrm{H} \alpha}$ regions display enhanced $\left[\mathrm{S}_{\mathrm{II}}\right] / \mathrm{H} \alpha,\left[\mathrm{N}_{\mathrm{II}}\right] / \mathrm{H} \alpha,[\mathrm{O}$ II] $/ \mathrm{H} \beta$ and $\left[\mathrm{O}_{\mathrm{I}}\right] / \mathrm{H} \alpha$. The gradients in these line ratios are determined by metallicity gradients and $\Sigma_{\mathrm{H} \alpha}$. In line ratio diagnostic diagrams, contamination by DIG moves $\mathrm{H}$ II regions towards composite or low-ionization nuclear emission-line region (LI(N)ER)-like regions. A harder ionizing spectrum is needed to explain DIG line ratios. Leaky $\mathrm{H}$ II region models can only shift line ratios slightly relative to $\mathrm{H}$ II region models, and thus fail to explain the composite/LI(N)ER line ratios displayed by DIG. Our result favours ionization by evolved stars as a major ionization source for DIG with LI(N)ER-like emission. DIG can significantly bias the measurement of gas metallicity and metallicity gradients derived using strong-line methods. Metallicities derived using $\mathrm{N} 2 \mathrm{O} 2$ are optimal because they exhibit the smallest bias and error. Using $\mathrm{O} 3 \mathrm{~N} 2, R_{23}$, $\mathrm{N} 2=[\mathrm{N} I I] / \mathrm{H} \alpha$ and $\mathrm{N} 2 \mathrm{~S} 2 \mathrm{H} \alpha$ to derive metallicities introduces bias in the derived metallicity gradients as large as the gradient itself. The strong-line method of Blanc et al. (IZI hereafter) cannot be applied to DIG to get an accurate metallicity because it currently contains only $\mathrm{H}$ II region models that fail to describe the DIG.
\end{abstract}

Key words: galaxies: abundances-galaxies: active-galaxies: evolution-galaxies: fundamental parameters-galaxies: ISM.

\section{INTRODUCTION}

Diffuse ionized gas (DIG hereafter) is an important gas component in star-forming galaxies. It was first identified in our Milky Way (MW) off the disc, and known as the Reynolds layer (Reynolds 1984). DIG is a major part of ionized gas in MW. In terms of mass, it is about 30 per cent of the MW neutral hydrogen (Reynolds 1990, 1991). DIG is also found in external galaxies both in extra-plannar haloes (e.g. Dettmar 1990; Rand, Kulkarni \& Hester 1990; Rand 1996; Hoopes, Walterbos \& Rand 1999; Rossa \& Dettmar 2003a,b) and in the disc (e.g. Monnet 1971; Zurita, Rozas \& Beckman 2000; Oey et al. 2007). The contribution of DIG to the total emission-line flux for face-on galaxies is substantial (e.g. Walterbos \& Braun 1994; Ferguson et al. 1996; Hoopes, Walterbos \& Greenawalt 1996; Greenawalt et al. 1998). 
For 109 star-forming galaxies in the SINGG sample, the DIG fraction in $\mathrm{H} \alpha$ flux is $0.59 \pm 0.19$, and this ratio depends on the $\mathrm{H} \alpha$ surface brightness of the whole galaxy (Oey et al. 2007). DIG is important in understanding the ionized gas in star-forming galaxies.

The differences between DIG and $\mathrm{H}$ II regions are not only in emission intensity, but also in emission-line ratios, indicating different physical conditions. The [S II] $\lambda \lambda 6717,6731 / \mathrm{H} \alpha,\left[\mathrm{N}_{\mathrm{II}}\right]$ $\lambda 6548 / \mathrm{H} \alpha,\left(\left[\mathrm{S}_{\mathrm{II}}\right] / \mathrm{H} \alpha\right.$ and $\left[\mathrm{N}_{\mathrm{II}}\right] / \mathrm{H} \alpha$ hereafter; e.g. Reynolds $1985 \mathrm{a}$; Hoopes \& Walterbos 2003; Madsen, Reynolds \& Haffner 2006), $\left[\mathrm{O}_{\mathrm{I}}\right] \lambda 6300 / \mathrm{H} \alpha$ and $\left[\mathrm{O}_{\mathrm{II}}\right] \quad \lambda 3727 / \mathrm{H} \beta \quad\left(\left[\mathrm{O}_{\mathrm{I}}\right] / \mathrm{H} \alpha\right.$ and $\left[\mathrm{O}_{\mathrm{II}}\right] / \mathrm{H} \beta$ hereafter, e.g. Haffner, Reynolds \& Tufte 1999; Voges \& Walterbos 2006) are found to be enhanced in DIG relative to $\mathrm{H}_{\mathrm{II}}$ regions. More quantitively, $\left[\mathrm{S}_{\mathrm{II}}\right] / \mathrm{H} \alpha,\left[\mathrm{N}_{\mathrm{II}}\right] / \mathrm{H} \alpha,\left[\mathrm{O}_{\mathrm{II}}\right] / \mathrm{H} \alpha,\left[\mathrm{O}_{\mathrm{I}}\right] / \mathrm{H} \alpha$ ratios correlate negatively with $\mathrm{H} \alpha$ flux in the MW and other galaxies (Reynolds et al. 1998; Haffner et al. 1999; Tüllmann \& Dettmar 2000; Hausen, Reynolds \& Haffner 2002; Madsen et al. 2006; Voges \& Walterbos 2006; Blanc et al. 2009). In high-spatial resolution observations (parsecs to tens of parsecs, such as those by the Wisconsin H-Alpha Mapper; WHAM), the line ratio versus $\Sigma_{\mathrm{H} \alpha}$ relation is a gradual transition from $\mathrm{H}$ in regions to DIG. In low-spatial resolution observations (hundreds of parsecs or kiloparsecs, such as integral-field studies of nearby galaxies), this relation is due to the mixing of $\mathrm{H}$ II regions and DIG within resolution elements. Different fractions of DIG and $\mathrm{H}$ II region contribution, combined with the different line ratios for these two types of regions would naturally produce the trend we see. Observed relations between line ratios and $\Sigma_{\mathrm{H} \alpha}$ provide an empirical method to separate DIG dominated and $\mathrm{H}$ II region-dominated regions (Blanc et al. 2009; Kaplan et al. 2016; Kreckel et al. 2016).

DIG line ratios cannot be explained by models of $\mathrm{H}$ II regions. On the classical BPT diagram (Baldwin, Phillips \& Terlevich 1981; Veilleux \& Osterbrock 1987; Kewley et al. 2001, 2006; Kauffmann et al. 2003), the location of an $\mathrm{H}$ II region is mainly determined by its metallicity and ionization parameter (e.g. Kewley \& Dopita 2002; Dopita et al. 2013). DIG shows a lower ionization parameter than $\mathrm{H}_{\text {II }}$ regions, which explains partly if not mostly the enhancement of $\left[\mathrm{N}_{\mathrm{II}}\right] / \mathrm{H} \alpha,\left[\mathrm{S}_{\mathrm{II}}\right] / \mathrm{H} \alpha$ and $\left[\mathrm{O}{ }_{\mathrm{II}}\right] / \mathrm{H} \alpha$ in DIG. However, just varying these two parameters cannot fully produce the line ratios seen in DIG (e.g. Galarza, Walterbos \& Braun 1999; Hoopes \& Walterbos 2003; Kaplan et al. 2016). A third variable is needed. Also, the line ratios of DIG within a galaxy often vary much more than those of $\mathrm{H}$ II regions. For example, in our MW, DIG $\left[\mathrm{S}_{\mathrm{II}}\right] / \mathrm{H} \alpha$ ratios display a dispersion of 0.13 (in linear space), compared with a dispersion of 0.03 for $\mathrm{H}$ II regions (Madsen et al. 2006). Temperature has been proposed as the strongest factor explaining the variations of DIG line ratios because it can explain the coherent variation of $[\mathrm{O}$ II] $\lambda 3727 / \mathrm{H} \alpha,[\mathrm{N} \mathrm{II}] \lambda 6548 / \mathrm{H} \alpha$ and $[\mathrm{S}$ II]/H $\alpha$ (Haffner et al. 1999; Mierkiewicz et al. 2006). The variation of temperature is a result of the balance between heating and cooling, which also requires a physical explanation. Besides photoionization, an additional source of heating might be important especially when the density is low (e.g. Reynolds \& Cox 1992). We will show in this paper that a harder ionizing spectrum could easily explain the DIG line ratios we observe in star-forming galaxies. The harder spectrum is capable of producing partially ionized regions that emit strong $\left[\mathrm{S} \mathrm{II}_{1}\right.$, [N II], $\left[\mathrm{O}_{\mathrm{II}}\right]$ and $\left[\mathrm{O}_{\mathrm{I}}\right]$, and an increase in temperature.

The DIG can impact our interpretation of the line-ratio diagnostic diagrams of galaxies for either integrated or spatially resolved spectroscopy. The BPT diagrams, for example, are widely used to diagnose the physical properties of ionized gas and separate different types of galaxies (Baldwin et al. 1981; Veilleux \&
Osterbrock 1987; Kewley et al. 2001, 2006; Kauffmann et al. 2003). When metallicity, ionization parameter, density or ionizing spectrum change, the location of ionized gas on the BPT diagram changes (e.g. Dopita et al. 2000, 2013; Kewley \& Dopita 2002; Kewley et al. 2013). When the nitrogen-to-oxygen abundance ratio (N/O) ratio is high, it is possible for a star-forming galaxy to be classified as composite galaxy (Pérez-Montero \& Contini 2009; Pérez-Montero et al. 2013, 2016). The enhanced $\left[\mathrm{N}_{\Pi}\right] / \mathrm{H} \alpha$ and $\left[\mathrm{S}_{\Pi]}\right] / \mathrm{H} \alpha$ of DIG would move the position of a star-forming galaxy towards the composite or low-ionization nuclear emission-line region $(\mathrm{LI}(\mathrm{N}) \mathrm{ER})$ region (e.g. Sarzi et al 2006; Stasińska et al. 2008; Kehrig et al. 2012; Yan \& Blanton 2012; Singh et al. 2013; Belfiore et al. 2016a,b; Gomes et al. 2016) on the BPT diagrams. Consequently, understanding DIG helps us understand the nature of galaxies classified as composites or $\mathrm{LI}(\mathrm{N}) \mathrm{ERs}$ by the BPT diagrams.

The study of DIG is also critical for gas-phase metallicity measurements in star-forming galaxies. Metallicity calibrations are generally based on $\mathrm{H}$ II region models that have certain assumptions. Some of these assumptions are not valid for DIG, and hence lead to biased metallicity measurements when DIG is present. The metallicity and ionization parameter $\left(q=\frac{\text { ionizing photon flux }}{N e}=U \times c\right)$ determine the line ratios of an $\mathrm{H}$ II region, and these two parameters are correlated (Dopita et al. 2006). Metallicity also determines the shape of the ionizing spectrum. In DIG, however, the correlation between $q$ and metallicity no longer holds, since DIG has a much lower ionization parameter than $\mathrm{H}$ II regions, and the ionizing spectrum shape can change. Given the very different line ratios such as $[\mathrm{N} \mathrm{II]} / \mathrm{H} \alpha$, $[\mathrm{O}$ III] $\lambda 5007 / \mathrm{H} \beta$ ([O III] $/ \mathrm{H} \beta$ hereafter) and [S II] $/ \mathrm{H} \alpha$ for DIG and $\mathrm{H}$ II regions, biases in metallicities derived from strong-line methods are inevitable. These biases potentially contribute to the large dispersion in metallicity measurements found in the literature. This will influence metallicity gradient measurements (Sánchez et al. 2014; Ho et al. 2015), metallicities at the outskirts of galaxies (Moran et al. 2012), the mass-metallicity relation in the local universe (e.g. McClure \& van den Bergh 1968; Lequeux et al. 1979; Garnett 2002; Tremonti et al. 2004; Lee et al. 2006) and at high redshift (Erb et al. 2006; Maiolino et al. 2008; Mannucci et al. 2009) and the mass-metallicity-SFR Fundamental Plane (Mannucci et al. 2010; Yates, Kauffmann \& Guo 2012; Andrews \& Martini 2013). For single-fibre surveys such as Sloan Digital Sky Survey (SDSS), the observed emission lines come from a combination of DIG and $\mathrm{H}$ II regions, and the bias introduced by DIG is uncertain and hard to quantify. With the help of integral field spectroscopy (IFS), we can study how the presence of DIG impacts metallicity measurements in detail.

In this paper, we demonstrate the prevalence of DIG in starforming galaxies from the Mapping Nearby Galaxies at APO (MaNGA) survey. The large sample and full optical wavelength coverage enable us to explore the optical line ratios for DIG for an unprecedented number of star-forming galaxies. Section 2 describes our sample and the emission-line measurements; while Section 3.1 shows the $[\mathrm{S} I \mathrm{I}] / \mathrm{H} \alpha$ versus $\Sigma_{\mathrm{H} \alpha}$ relation that illustrates the dominance of DIG in low $\Sigma_{\mathrm{H} \alpha}$ regions. Section 3 demonstrates how DIG impact line ratios like $\left[\mathrm{S}_{\mathrm{II}}\right] / \mathrm{H} \alpha,\left[\mathrm{N}_{\mathrm{II}}\right] / \mathrm{H} \alpha,\left[\mathrm{O}_{\mathrm{II}}\right] / \mathrm{H} \beta,\left[\mathrm{O}_{\mathrm{I}}\right] / \mathrm{H} \alpha$, $\left[\mathrm{O}\right.$ III] $/\left[\mathrm{O}_{\mathrm{II}}\right],[\mathrm{O} \mathrm{III}] / \mathrm{H} \beta$. Section 4 tests the leaky $\mathrm{H}$ II region model as well as one in which hot evolved stars serve as the ionization source of the DIG, using different line ratios and diagnostic diagrams. Section 5 presents our study of how the DIG impacts metallicity derived using strong-line methods: $\mathrm{N} 2, \mathrm{~N} 2 \mathrm{O} 2, R_{23}, \mathrm{O} 3 \mathrm{~N} 2$ and $\mathrm{N} 2 \mathrm{~S} 2 \mathrm{H} \alpha$ and IZI. Section 6 contains a discussion of these results, summarized then in Section 7. We use a cosmology with $H_{0}=70 \mathrm{~km} \mathrm{~s}^{-1} \mathrm{Mpc}^{-1}$, $\Omega_{\mathrm{m}}=0.3$, and $\Omega_{\Lambda}=0.7$ throughout this paper. 


\section{SAMPLE AND MEASUREMENTS}

\subsection{MaNGA overview}

MaNGA (Bundy et al. 2015) is one of the three core programs in the Sloan Digital Sky Survey-IV (SDSS-IV). It aims at obtaining IFS of 10000 nearby galaxies. The survey employs the BOSS spectrographs (Smee et al. 2013) on the $2.5 \mathrm{~m}$ Sloan Foundation Telescope (Gunn et al. 2006). The spectrographs provide a spectral coverage from 3600 to $10300 \AA$ at a resolution around $R \sim 2000$. The fibre feed system has been re-designed (Drory et al. 2015) to accommodate 1423 fibers that are bundled into five 127-fibre bundles, two 91-fibre bundles, four 61-fibre bundles, four 37-fibre bundles, two 19-fibre bundles, twelve 7-fibre mini-bundles and 92 sky fibers. All bundles with 19 or more fibers are used for galaxy targets, while the mini-bundles are used to observe standard stars providing a flux calibration to an accuracy better than 5 per cent (Yan et al. 2016b). Each individual fibre is 2 arcsec in diameter. The filling factor of fibers in the bundles is 56 per cent. The observations are done with a dithering scheme to achieve a complete spatial coverage and near critical sampling of the point spread function (PSF; Law et al. 2015).

The MaNGA sample is designed to have a roughly flat $i$-band absolute magnitude distribution (Wake et al., in preparation). The reconstructed data cubes have 2.5 arcsec PSF and 0.5 arcsec spaxel (Law et al. 2015, 2016; Yan et al. 2016a,b). The sample has a redshift range of $0.01<z<0.15$, meaning that the targets cover a factor of 15 in distance, and consequently the physical resolution also span a range of 15 . We select more luminous galaxies that are larger, at higher redshift. The typical physical resolution is 1-2 kpc. The physical resolution is highly correlated with luminosity, which means that we need to be aware that any results we find that might depend on physical resolution will be specific to a specific range of luminosity and vice versa. The primary sample, which covers to 1.5 effective radius $\left(R_{\mathrm{e}}\right)$ in major axis, comprises two-third of the sample, while the secondary sample, which covers to $2.5 R_{\mathrm{e}}$, comprises the remaining one-third. Massive galaxies, which are bigger, are selected at higher redshift. All plates are exposed until we reach an $(\mathrm{S} / \mathrm{N})^{2}$ of 20 per pixel per fibre in the $g$-band continuum for a galactic extinction-corrected $g$-band fibre magnitude of 22 , and an $(\mathrm{S} / \mathrm{N})^{2}$ of 36 per pixel per fibre in the $i$-band continuum for a galactic extinction-corrected $i$-band fibre magnitude of 21 (Yan et al. 2016a). MaNGA provides a benchmark of resolved ionized gas properties, stellar population and dynamical evolution in the local universe (e.g. Belfiore et al. 2015; Li et al. 2015; Wilkinson et al. 2015).

\subsection{Sample selection}

In this paper, we use a sample of 81 regular survey plates observed before the summer of 2015. There are 1391 unique galaxies observed. This corresponds to the sample that was released in DR13. The photometry data are from NASA-Sloan Atlas catalogue ${ }^{1}$ (NSA Catalogue). By applying a colour cut of $M_{u}-M_{r}<2.1\left(M_{u}\right.$ and $M_{r}$ are rest-frame absolute magnitudes without extinction correction), we select only blue galaxies. This resulted in a sample of 592 galaxies. To remove edge-on galaxies, we apply the cut $b / a>0.5$, where $b$ and $a$ are the minor and major axis of the Sérsic model. Edge-on galaxies suffer from strong projection effects and are also prone to severe extinction. These criteria leave us with 365 galaxies. Active galactic nuclei (AGNs) are not eliminated because we care about DIG that is far away from the centre. From this sample, we choose three galaxies that have large internal variations of $\mathrm{H} \alpha$ surface brightness and best spatial resolution (127 fibre IFUs) to demonstrate the impact of DIG on line ratios, interpretation of diagnostic diagrams and metallicity measurements. We discuss in Sections 3.4 and 5.7 that the impact of DIG is prevalent in all star-forming galaxies in our sample.

\subsection{Spectrum fitting}

We start with the data cubes produced by the MaNGA data reduction pipeline (Law et al. 2016). We first construct Voronoi bins of the spectra by requiring the $\mathrm{S} / \mathrm{N}$ in $r$ band in each bin to be greater than $30 \AA^{-1}$. The covariance between spaxels is not accounted for when binning. With $\mathrm{BCO}^{2}$ (Bruzual \& Charlot 2003), we produce 14 continuum templates for simple stellar populations (SSPs) with $Z_{*}=0.02$ and 0.008 and ages $=13,7,2,1,0.5,0.25$ and $0.125 \mathrm{Gyr}$. For the stacked spectrum in each bin, we fit combinations of these SSPs, fitting velocity and relative amplitudes to spectra combined from spaxels in a Voronoi bin. We then derive the stellar velocity dispersion using the vdispfit.pro in IDLSPEC2D ${ }^{3}$ package. $20 \AA$ windows around [O II] $\lambda 3727, \mathrm{H} \beta,[\mathrm{O}$ III] $\lambda 4959,[\mathrm{O} \mathrm{III}] \lambda 5007$, [O I $\left.] \lambda 6300,\left[\mathrm{~N}_{\mathrm{II}}\right] \lambda 6548, \mathrm{H} \alpha, \mathrm{N}_{\mathrm{II}}\right] \lambda 6583$ and [S II] $\lambda \lambda 6717,6731$ are masked during the continuum fitting. We smooth the observed spectrum with a 50 pixel window and then subtract the smoothed spectrum from the original spectrum. This smoothing basically removes remaining spectrophotometric calibration mismatch between data and templates as well as effects of internal extinction. The smoothing scale is $\sim 3000 \mathrm{~km} \mathrm{~s}^{-1}$. By doing this, we are left with only small-scale spectral variations such as absorption lines and emission lines, which we refer to as the 'line feature spectrum'. The same technique is applied to the SSP templates to get the 'line feature templates' that only contains small-scale information. A linear regression is performed to fit the 'line feature spectrum' with the sum of 'line feature templates' at a number of velocity offsets relative to the systematic redshift of the galaxy. The velocity grid ranges from -450 to $450 \mathrm{~km} \mathrm{~s}^{-1}$ with an interval of $30 \mathrm{~km} \mathrm{~s}^{-1}$. For each Voronoi bin at each velocity, the least-square fitting yields a $\chi^{2}$ for that fit. A quadratic curve is fitted to the $\chi^{2}$ versus velocity curve to find the velocity offset yielding the minimum $\chi^{2}$. For each spaxel in one Voronoi bin, we use the fitted stellar continuum, the combination of $14 \mathrm{BC} 03$ templates, as one template, and adjust the amplitude to fit the continuum in each spaxel so that we can measure the emission-line ratios on a spaxel by spaxel basis. The residual emission line-only spectrum is stored for refined emissionline fitting. We use single Gaussians to fit the [O I], $\mathrm{H} \beta$, [O III], [N II] $\lambda 6548, \mathrm{H} \alpha,\left[\mathrm{N}_{\mathrm{II}}\right] \lambda 6583$ and $\left[\mathrm{S}_{\mathrm{II}}\right]$, respectively, and we use two Gaussians to fit [O II]. The line ratio of [ $\left.\mathrm{N}_{\text {II }}\right] \lambda 6583 /\left[\mathrm{N}_{\text {II }}\right] \lambda 6548$ is fixed to 3 . Velocity dispersions and central velocity shifts of all emission lines are tied to be the same. The $\mathrm{H} \alpha$ surface brightness maps for the three representative galaxies are shown in Fig. 1. The errors of the line strength are obtained by the MPFIT package which only includes the fitting errors (Markwardt 2009). An S/N cut of 5 is applied to emission lines used.

\footnotetext{
${ }^{2}$ http://www2.iap.fr/users/charlot/bc2003/

${ }^{3}$ http://spectro.princeton.edu/idlspec2d_doc.html
} 

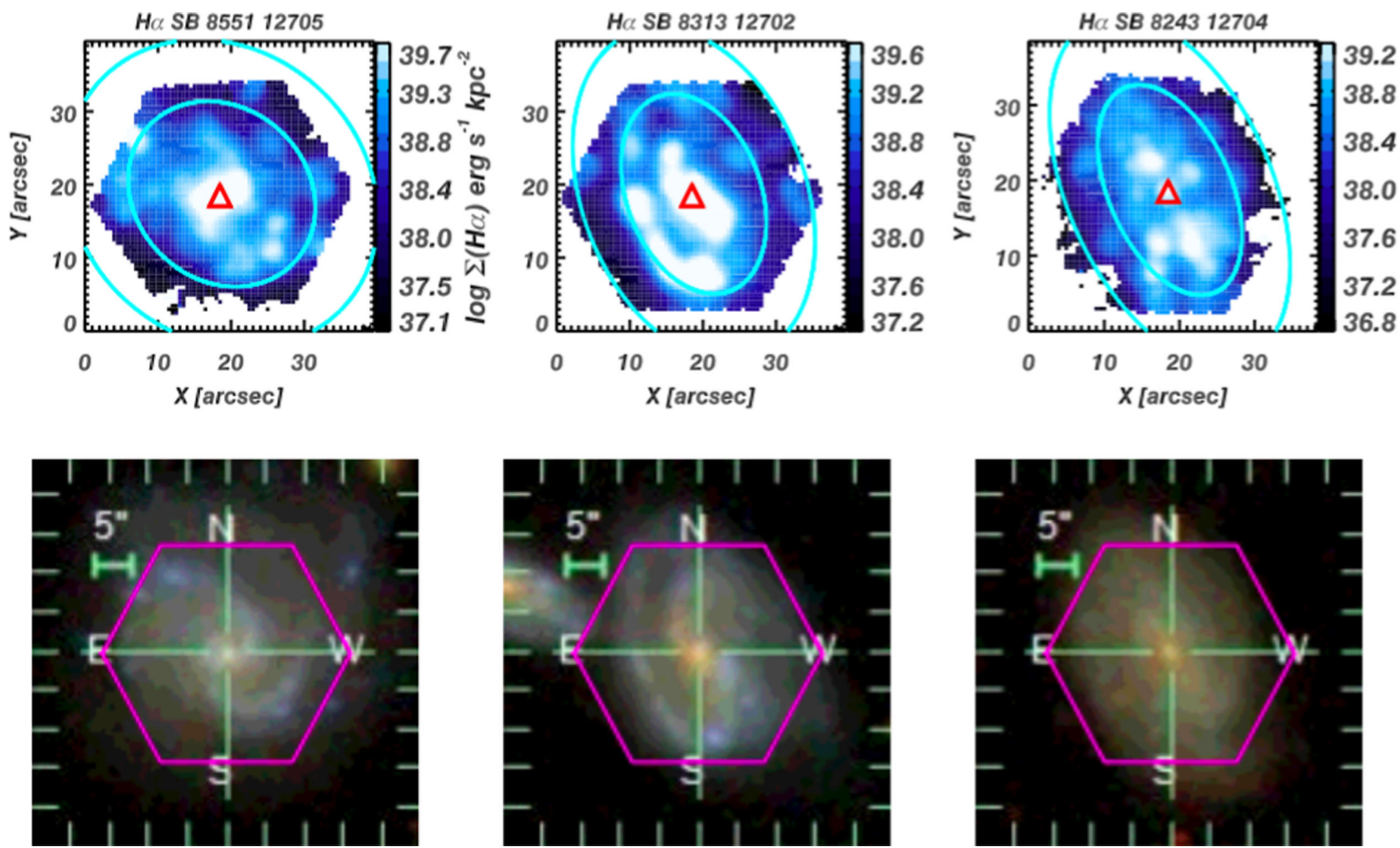

Figure 1. $\mathrm{H} \alpha$ surface brightness plot and SDSS optical image for each galaxy. The cyan ellipses in the upper represent are 1.5 and 2.5 times the effective radius. The hexagons in the lower row demonstrate the location of the IFU bundles on the object.
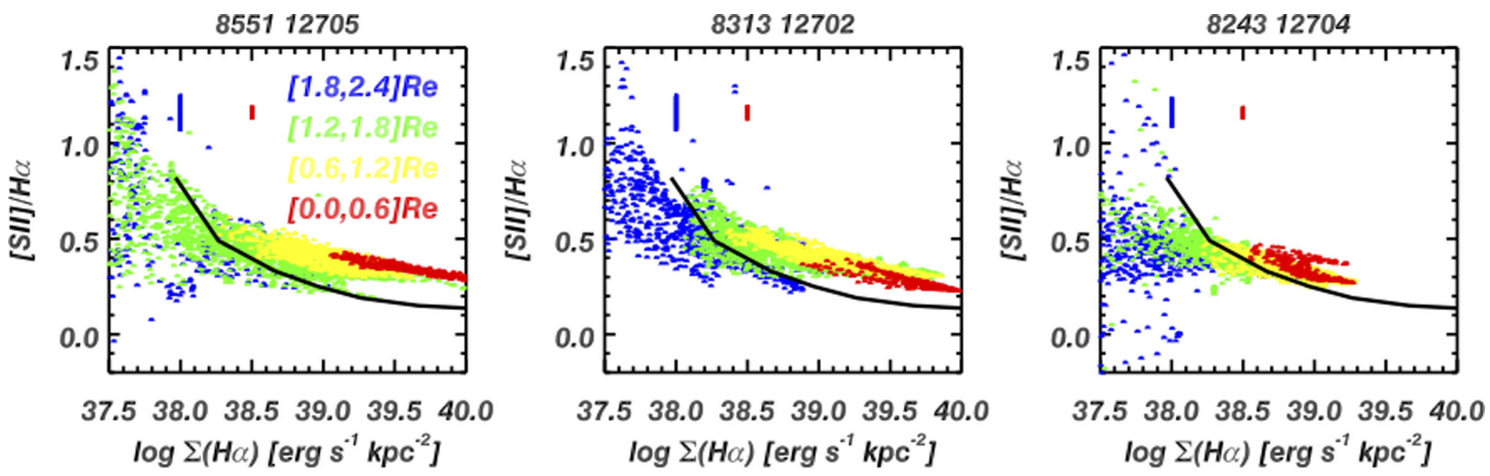

Figure 2. $\left[\mathrm{S}_{\text {II }}\right] / \mathrm{H} \alpha$ versus $\mathrm{H} \alpha$ surface brightness plot for each galaxy. Different colour represent different annuli. The solid line is the $\left[\mathrm{S}_{\mathrm{II}}\right] / \mathrm{H} \alpha$ versus $\mathrm{H} \alpha$ surface brightness relation for MW (Madsen et al. 2006). We see that high- and low-H $\alpha$ surface brightness regions have different $[\mathrm{S}$ II]/H $\alpha$. The red and blue bars show the typical error at $\log \Sigma_{\mathrm{H} \alpha}=38.5$ and $38 \mathrm{erg} \mathrm{s}^{-1} \mathrm{kpc}^{-2}$ for individual spaxels. The length of the error bars is $2 \sigma$.

\section{VARIATION OF LINE RATIOS WITH H $\alpha$ SURFACE BRIGHTNESS}

\subsection{Separation of regions dominated by DIG and $\mathrm{H}$ II regions}

The first step to study DIG is separating DIG from $\mathrm{H}$ II regions. The best way to isolate DIG is to identify individual $\mathrm{H}$ II regions and subtract them out (e.g. Walterbos \& Braun 1994; Zurita et al. 2000, 2002; Thilker et al. 2002; Sánchez et al. 2012). Due to the limited spatial resolution of MaNGA, we cannot resolve individual $\mathrm{H}$ II regions in one galaxy. Our reconstructed PSF is about 2.5 arcsec full width at half-maximum $(2 \operatorname{arcsec}$ covers $1 \mathrm{kpc}$ at $z=0.025$ ) while the size of a typical $\mathrm{H}$ II region is a few to hundreds of parsecs (Kennicutt 1984; Garay \& Lizano 1999; Kim \& Koo 2001; Hunt \& Hirashita 2009). So the light in one spaxel is always a mixture of $\mathrm{H}$ II region emission and the surrounding DIG. Instead, we separate DIG-dominated regions and $\mathrm{H}$ II regiondominated regions. In Fig. 2, we see that the low-surface brightness regions have $[\mathrm{S} \Pi] / \mathrm{H} \alpha \sim 0.5-1.0$, while the high-surface brightness regions show $\left[\mathrm{S}_{\mathrm{II}}\right] / \mathrm{H} \alpha \sim 0.2-0.4$, depending on the metallicity. Since $[\mathrm{S}$ II] $/ \mathrm{H} \alpha$ is sensitive to metallicity, and there are metallicity gradients in galaxies, we separate all the spaxels in each galaxy into different radial bins: [0,0.6],[0.6,1.2], [1.2,1.8] and [1.8,2.4] $R_{\mathrm{e}}$. The $[\mathrm{S}$ II $] / \mathrm{H} \alpha$ versus $\Sigma_{\mathrm{H} \alpha}$ relations are similar in all radial bins, meaning that the variation of $\left[\mathrm{S}_{\mathrm{II}}\right] / \mathrm{H} \alpha$ is not caused by metallicity variation, but reflects the transition from DIG-dominated low $\Sigma_{\mathrm{H} \alpha}$ regions to $\mathrm{H}$ II region-dominated high $\Sigma_{\mathrm{H} \alpha}$ regions. This figure illustrates that $\Sigma_{\mathrm{H} \alpha}$ can be used to separate the two different kinds of regions: low $\Sigma_{\mathrm{H} \alpha}$ DIG-dominated regions and high $\Sigma_{\mathrm{H} \alpha} \mathrm{H}_{\text {II }}$ region-dominated regions. 

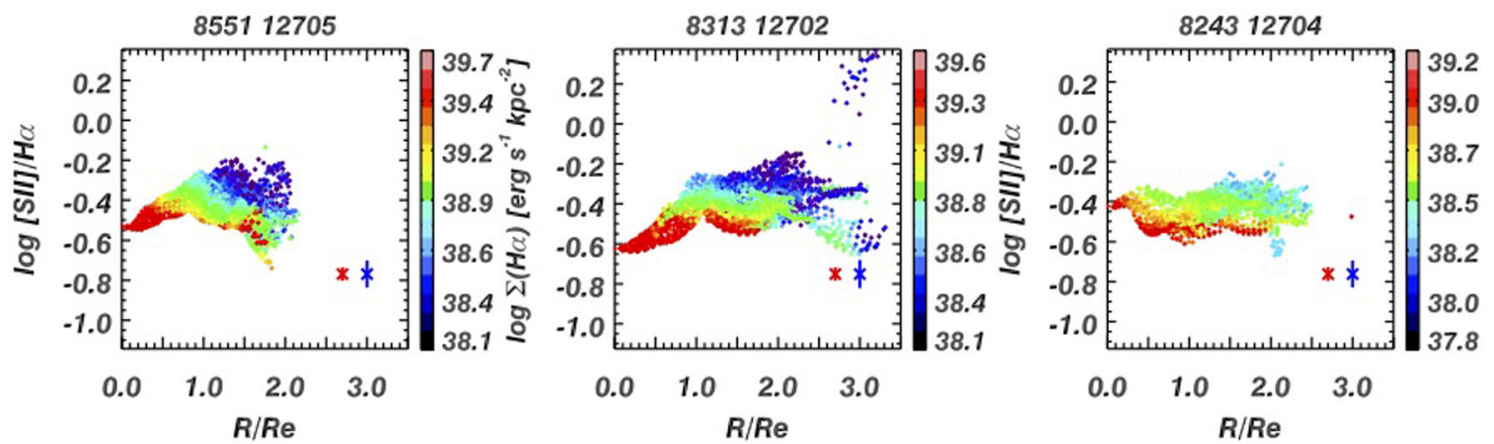

Figure 3. $[\mathrm{S} \mathrm{II}] / \mathrm{H} \alpha$ as a function of radius. The dispersion at fixed radius is about 0.2 dex. But when the dots are colour-coded by H $\alpha$ surface brightness as shown in the colourbar, we see a beautiful rainbow pattern. The dispersion is significantly reduced at fixed $\Sigma_{\mathrm{H} \alpha}$. This is because DIG that dominates the low $\Sigma_{\mathrm{H} \alpha}$ region has higher $\left[\mathrm{S}_{\mathrm{II}}\right] / \mathrm{H} \alpha$. The red and blue bars show the typical line ratio error at $\log \Sigma_{\mathrm{H} \alpha}=39$ and $38.5 \mathrm{erg} \mathrm{s}^{-1} \mathrm{kpc}^{-2}$ for individual spaxels. The length of the error bars is $2 \sigma$. We show in Fig. 11 that the impact of DIG is prevalent in all star-forming galaxies in our sample, not only in the three galaxies we show.
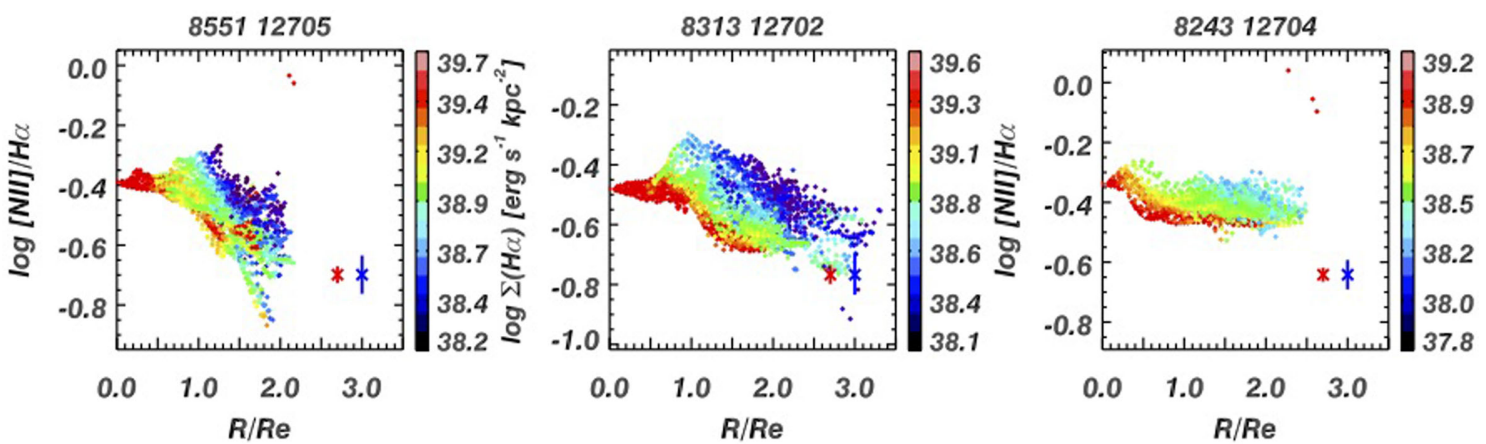

Figure 4. $\left[\mathrm{N}_{\mathrm{II}}\right] / \mathrm{H} \alpha$ as a function of radius. We see $\left[\mathrm{N}_{\mathrm{II}}\right] / \mathrm{H} \alpha$ generally drops towards large radius due to a metallicity gradient. The dispersion at fixed radius is about 0.2 dex. But when the dots are colour-coded by $\mathrm{H} \alpha$ surface brightness as shown in the colourbar, we see a beautiful rainbow pattern. This shows $[\mathrm{N}$ II] $] / \mathrm{H} \alpha$ is enhanced in DIG. The red and blue bars show the typical line ratio error at $\log \Sigma_{\mathrm{H} \alpha}=39$ and $38.5 \mathrm{erg} \mathrm{s}^{-1} \mathrm{kpc}^{-2}$ for individual spaxels. The length of the error bars is $2 \sigma$.

\section{$3.2[\mathrm{~S} \mathrm{II}] / \mathrm{H} \alpha,[\mathrm{N} \mathrm{II}] / \mathrm{H} \alpha,[\mathrm{O} \mathrm{II}] / \mathrm{H} \beta$ and $[\mathrm{O} \mathrm{I}] / \mathrm{H} \alpha$}

Galaxies show metallicity variations, in particular, in the form of radial gradients (e.g. Sánchez et al. 2014; Ho et al. 2015). It is crucial to control metallicity before exploring how the line ratios vary with other parameters like $\mathrm{H} \alpha$ surface brightness because metallicity is a major source of variation in line ratios. We plot line ratios as a function of radius, and colour code the dots with $\mathrm{H} \alpha$ surface brightness. We assume that the metallicity is constant within annuli for each galaxy but may be changing with radius.

We explore the impact of DIG on $\left[\mathrm{S}_{\mathrm{II}}\right] / \mathrm{H} \alpha$ first. We colour-code the dots by $\mathrm{H} \alpha$ surface brightness and show how $\left[\mathrm{S}_{\mathrm{II}}\right] / \mathrm{H} \alpha$ changes with radius in Fig. 3. At a fixed radius, we see a rainbow pattern such that low $\Sigma_{\mathrm{H} \alpha}$ regions have higher $\left[\mathrm{S}_{\mathrm{II}}\right] / \mathrm{H} \alpha$. This is a direct demonstration that $[\mathrm{S} \mathrm{II}] / \mathrm{H} \alpha$ is enhanced in DIG after controlling for metallicity.

We then explore how DIG impacts $\left[\mathrm{N}_{\mathrm{II}}\right] / \mathrm{H} \alpha .\left[\mathrm{N}_{\mathrm{II}}\right] / \mathrm{H} \alpha$ is used as a metallicity indicator since nitrogen is a secondary element and proportional to $Z^{2}$ while $\mathrm{H} \alpha$ is not sensitive to metallicity (e.g. Storchi-Bergmann, Calzetti \& Kinney 1994; van Zee, Salzer \& Haynes 1998; Denicoló, Terlevich \& Terlevich 2002). We colourcode the dots by $\mathrm{H} \alpha$ surface brightness and show how $\left[\mathrm{N}_{\text {II }}\right] / \mathrm{H} \alpha$ changes with radius in Fig. 4. If we control for $\mathrm{H} \alpha$ surface brightness by looking at the dots with the same colour, $[\mathrm{N}$ II $] / \mathrm{H} \alpha$ decreases towards large radius, reflecting a metallicity gradient. Due to the presence of an $\mathrm{H} \alpha$ surface brightness gradient, the different surface brightness bins usually trace different parts of the galaxy. However, at the radius where they overlap, we see a rainbow pattern. At a fixed radius, DIG-dominated low-surface brightness region shows a higher $\left[\mathrm{N}_{\mathrm{II}}\right] / \mathrm{H} \alpha$. If we use $\mathrm{N} 2$ to derive the metallicity, the enhancement means that the metallicity would be overestimated in those spaxels with a high DIG fraction and vice versa. The impact of DIG on the metallicity derived using $\left[\mathrm{N}_{\mathrm{II}}\right] / \mathrm{H} \alpha$ is given in Section 5.1.

We show how $\left[\mathrm{O}_{\mathrm{II}}\right] / \mathrm{H} \beta$ changes with radius and $\mathrm{H} \alpha$ surface brightness in Fig. 5. The extinction correction is not applied because it is not reliable when the emission line, especially $\mathrm{H} \beta$, is weak. Besides, a foreground dust screen may not be the appropriate model for DIG. Finally, We show in Fig. 6 that $\mathrm{H} \beta / \mathrm{H} \alpha$ does not depend on $\mathrm{H} \alpha$ surface brightness, meaning that extinction will not produce any line ratios change between DIG and $\mathrm{H}$ II regions.

$[\mathrm{O} \mathrm{II}] / \mathrm{H} \beta$ generally increases with radius at fixed $\mathrm{H} \alpha$ surface brightness due to a metallicity gradient. At fixed radius, the low$\mathrm{H} \alpha$ surface brightness regions have higher $[\mathrm{O} \mathrm{II}] / \mathrm{H} \beta$. It is interesting that $\left[\mathrm{O}_{\mathrm{II}}\right] / \mathrm{H} \beta$ and $[\mathrm{N} I] / \mathrm{H} \alpha$ both increase with decreasing surface brightness at fixed radius while they change reversely with radius at fixed $\mathrm{H} \alpha$ surface brightness. The opposite variation trends of $[\mathrm{O} I \mathrm{II}] / \mathrm{H} \beta$ and $\left[\mathrm{N}{ }_{\mathrm{II}}\right] / \mathrm{H} \alpha$ with radius at fixed surface brightness are consistent with a metallicity variation. With $[\mathrm{O} / \mathrm{H}]$ above solar value, $[\mathrm{N} I] / \mathrm{H} \alpha$ increases with metallicity due to the addition of secondary Nitrogen while $[\mathrm{O}$ II $] / \mathrm{H} \beta$ drops with increasing metallicity because of decreasing temperature. The positive correlation between $[\mathrm{O}$ II $] / \mathrm{H} \beta$ and $\left[\mathrm{N}_{\mathrm{II}}\right] / \mathrm{H} \alpha$ with surface brightness at fixed radius is seemingly consistent with temperature variation. Mierkiewicz 

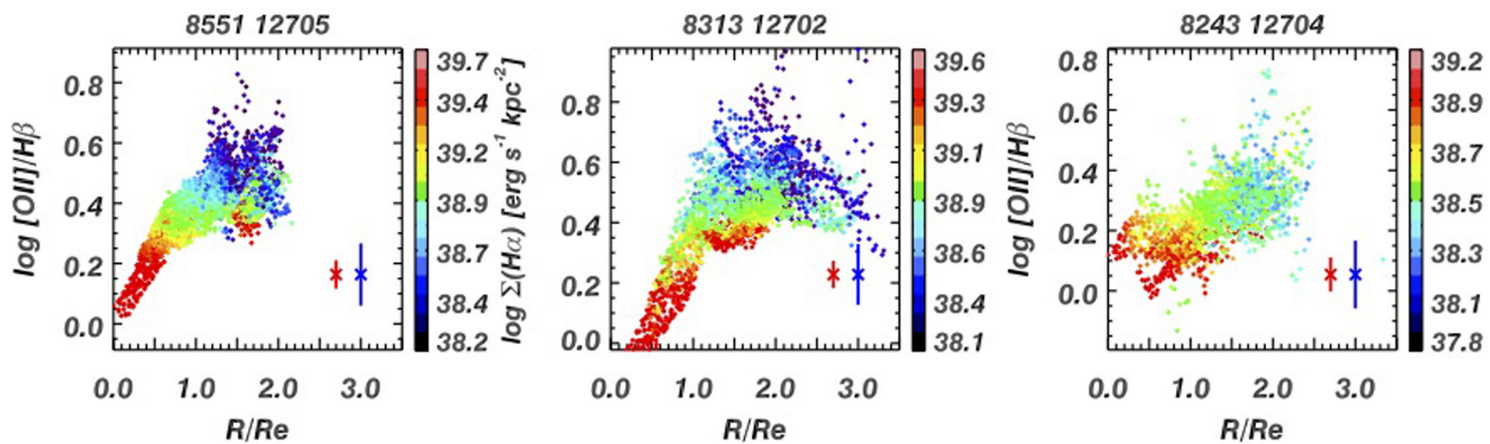

Figure 5. $\left[\mathrm{O}_{\mathrm{II}}\right] / \mathrm{H} \beta$ as a function of radius. The dots are colour-coded by $\Sigma_{\mathrm{H} \alpha}$. We see rainbow patterns as in Fig. 4 , indicating that $[\mathrm{O}$ II $] / \mathrm{H} \beta$ is enhanced in DIG. The red and blue bars show the typical line ratio error at $\log \Sigma_{\mathrm{H} \alpha}=39$ and $38.5 \mathrm{erg} \mathrm{s}^{-1} \mathrm{kpc}^{-2}$ for individual spaxels. The length of the error bars is $2 \sigma$.
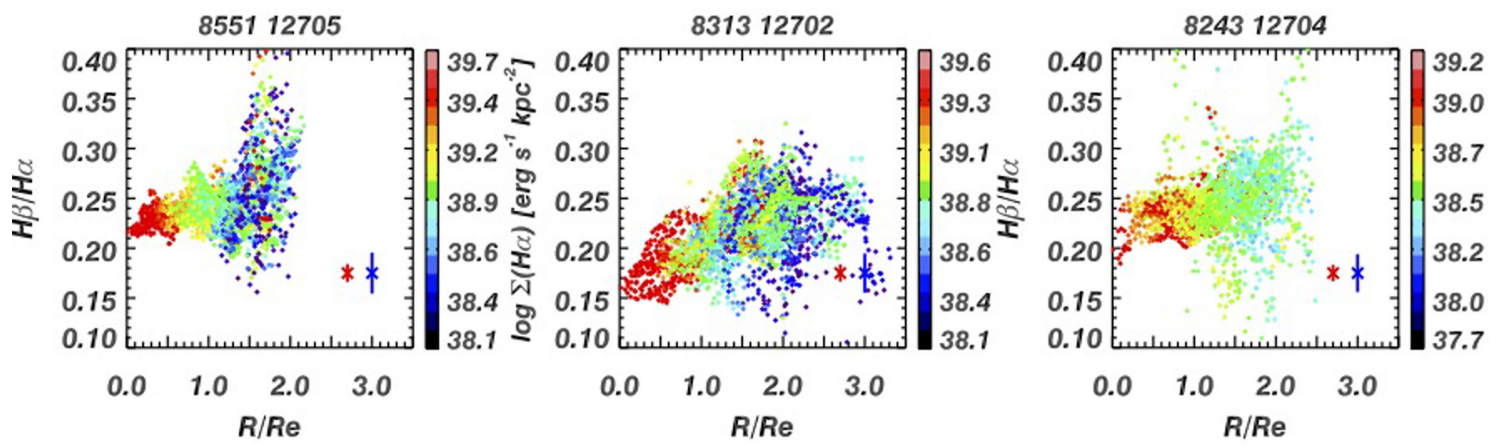

Figure 6. $\mathrm{H} \beta / \mathrm{H} \alpha$ as a function of radius. The dots are colour-coded by $\Sigma_{\mathrm{H} \alpha}$. We don't see any dependence of $\mathrm{H} \beta / \mathrm{H} \alpha$ on $\Sigma_{\mathrm{H} \alpha}$ at a given radius, implying the extinction correction is not the reason for the different line ratios of DIG and $\mathrm{H}_{\mathrm{II}}$ regions. The red and blue bars show the typical line ratio error at $\log \Sigma_{\mathrm{H} \alpha}=$ $39 \mathrm{erg} \mathrm{s}^{-1} \mathrm{kpc}^{-2}$ and $\log \Sigma_{\mathrm{H} \alpha}=38.5 \mathrm{erg} \mathrm{s}^{-1} \mathrm{kpc}^{-2}$ for individual spaxel. The length of the error bars is $2 \sigma$.

et al. (2006) show that $[\mathrm{O}$ II $] / \mathrm{H} \alpha$ and $[\mathrm{N} \mathrm{II}] / \mathrm{H} \alpha$ correlate positively with temperature variation and they concluded that the variation of line ratios is driven by temperature variations (Haffner et al. 1999; Haffner et al. 2009). However, the variation of temperature is a result of the balance between heating and cooling, which itself needs a physical explanation. Besides, some significant assumptions need to be made to use $\left[\mathrm{O}_{\mathrm{II}}\right] / \mathrm{H} \beta$ and $\left[\mathrm{N}_{\mathrm{II}}\right] / \mathrm{H} \alpha$ as temperature tracers. The biggest assumption is that [O II] and $\mathrm{H} \beta$ are cospatial. However, we only resolve galaxies to kiloparsec scale for MaNGA survey, thus we see the integrated emission from all layers of interstellar medium (ISM) around an ionizing source. The co-spatial assumption may not hold for DIG. [N II]/H $\alpha$ line ratios are often used as a metallicity indicator for extragalactic studies, while it may be used as a temperature indicator for galactic uses. Both metallicity and temperature are relevant to $[\mathrm{N}$ II] $/ \mathrm{H} \alpha$ emission. Finally, a decrease in ionization parameter and/or a harder ionizing spectrum towards lower $\Sigma_{\mathrm{H} \alpha}$ will also translate into enhancement of $[\mathrm{O} I \mathrm{II}] / \mathrm{H} \beta$ and $[\mathrm{N} \mathrm{II}] / \mathrm{H} \alpha$. This is further explored in Section 4.

[O I] is an important line to diagnose the physical properties of ionized gas (Dopita et al. 2000; Kewley et al. 2006). [O I] is detected in DIG of M33 and [O I] $/ \mathrm{H} \alpha$ is found to correlate negatively with emission measure (Voges \& Walterbos 2006). [O I $/ \mathrm{H} \alpha$ is a strong function of temperature and the hardness of the ionizing spectrum. [O I] has a high critical density $\left(n_{\left[\mathrm{O}_{\mathrm{I}}\right]}=10^{6.3} \mathrm{~cm}^{-3}\right)$, thus it is emitted in high-density neutral and partially ionized regions. $\left[\mathrm{O}_{\mathrm{I}}\right] / \mathrm{H} \alpha$ is also an excellent tracer of shocks (Dopita et al. 2000; Kewley \& Dopita 2002; Allen et al. 2008). In Fig. 7, we show how the $\left[\mathrm{O}_{\mathrm{I}}\right] / \mathrm{H} \alpha$ changes with $\mathrm{H} \alpha$ surface brightness and radius for our three galaxies. For all galaxies, $\left[\mathrm{O}_{\mathrm{I}}\right] / \mathrm{H} \alpha$ increases with radius. At a fixed radius, $\left[\mathrm{O}_{\mathrm{I}}\right] / \mathrm{H} \alpha$ is enhanced for low-surface brightness regions, indicating $\left[\mathrm{O}_{\mathrm{I}}\right] / \mathrm{H} \alpha$ is higher in DIG. We note that the contrast between DIG and $\mathrm{H}$ II region is large; [O I]/H $\alpha$ drops $\sim 1$ dex with an increase of 1 dex in $\Sigma_{\mathrm{H} \alpha}$.

\section{$3.3[\mathrm{O} \mathrm{III}] /[\mathrm{O}$ II $]$ and $[\mathrm{O} \mathrm{III}] / \mathrm{H} \beta$}

$[\mathrm{O}$ III $] /\left[\mathrm{O}_{\mathrm{II}}\right]$ is a good ionization parameter proxy when metallicity is controlled (Kewley \& Dopita 2002, see also Fig. 18). We show how the $\left[\mathrm{O}_{\mathrm{III}}\right] /\left[\mathrm{O}_{\mathrm{II}}\right]$ values of low-surface brightness spaxels differ from the high-surface brightness spaxels for our sample galaxies in Fig. 8. This relation has large dispersion but there is a clear trend. At a fixed $\mathrm{H} \alpha$ surface brightness, [O $\mathrm{III}] /\left[\mathrm{O}_{\mathrm{II}}\right]$ increases as we go to larger radii due to a metallicity gradient (see also Fig. 14). At a fixed radius, $\left[\mathrm{O}_{\mathrm{III}}\right] /\left[\mathrm{O}_{\mathrm{II}}\right]$ mostly decreases with decreasing surface brightness. The result suggests that DIG has a lower ionization parameter than $\mathrm{H}$ II regions.

$[\mathrm{O} \mathrm{III}] / \mathrm{H} \beta$ is another frequently used ionization parameter proxy. Its dependence on metallicity and ionizing spectrum hardness is much stronger than $\left[\mathrm{O}_{\mathrm{III}}\right] /\left[\mathrm{O}_{\mathrm{II}}\right]$. How $\left[\mathrm{O}_{\mathrm{III}}\right] / \mathrm{H} \beta$ of DIG differs from that of $\mathrm{H}$ II regions depends on the specific physical properties of the ISM. DIG can show either higher (Wang, Heckman \& Lehnert 1997; Rand 1998, 2000; Collins \& Rand 2001; Otte 2001, 2002; Otte, Gallagher \& Reynolds 2002) or lower (MW: Reynolds 1985b; M31: Greenawalt, Walterbos \& Braun 1997; Galarza et al. 1999) $\left[\mathrm{O}\right.$ III]/H $\beta$ than $\mathrm{H}_{\text {II }}$ regions. The study of our whole sample of 395 galaxies in Section 3.4 confirms the diverse behaviour of $[\mathrm{O} I I] / \mathrm{H} \beta$ in DIG. In the previous paragraph, we have 

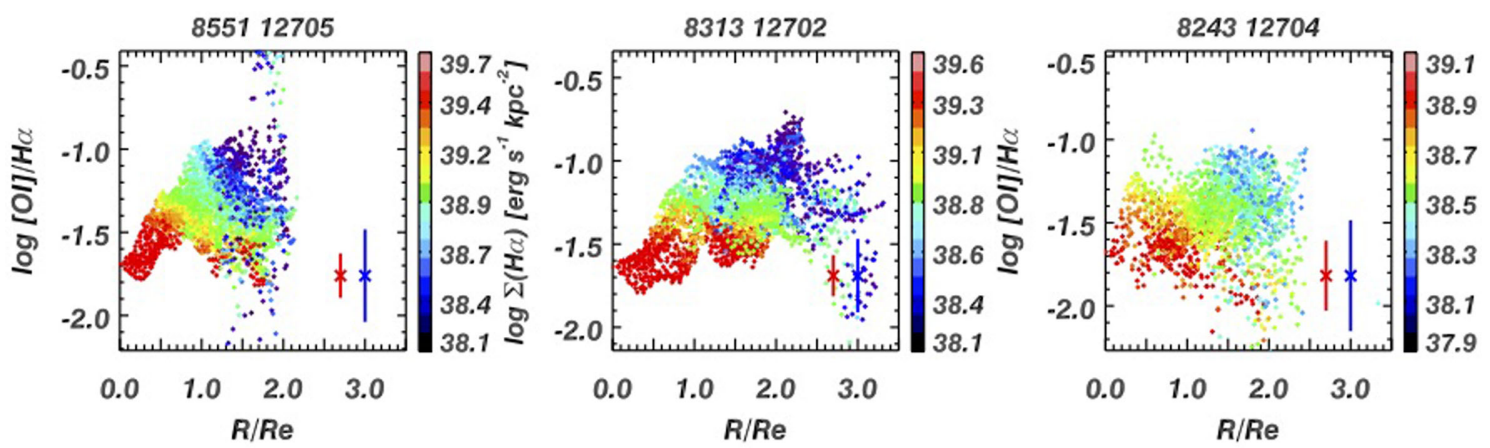

Figure 7. $\left[\mathrm{O}_{\mathrm{I}}\right] / \mathrm{H} \alpha$ as a function of radius. The dots are colour-coded by $\Sigma_{\mathrm{H} \alpha}$. We see rainbow patterns as in Fig. 4, indicating that [O I $] / \mathrm{H} \alpha$ is enhanced in DIG. The red and blue bars show the typical line ratio error at $\log \Sigma_{\mathrm{H} \alpha}=39$ and $38.5 \mathrm{erg} \mathrm{s}^{-1} \mathrm{kpc}^{-2}$ for individual spaxels. The length of the error bars is $2 \sigma$.
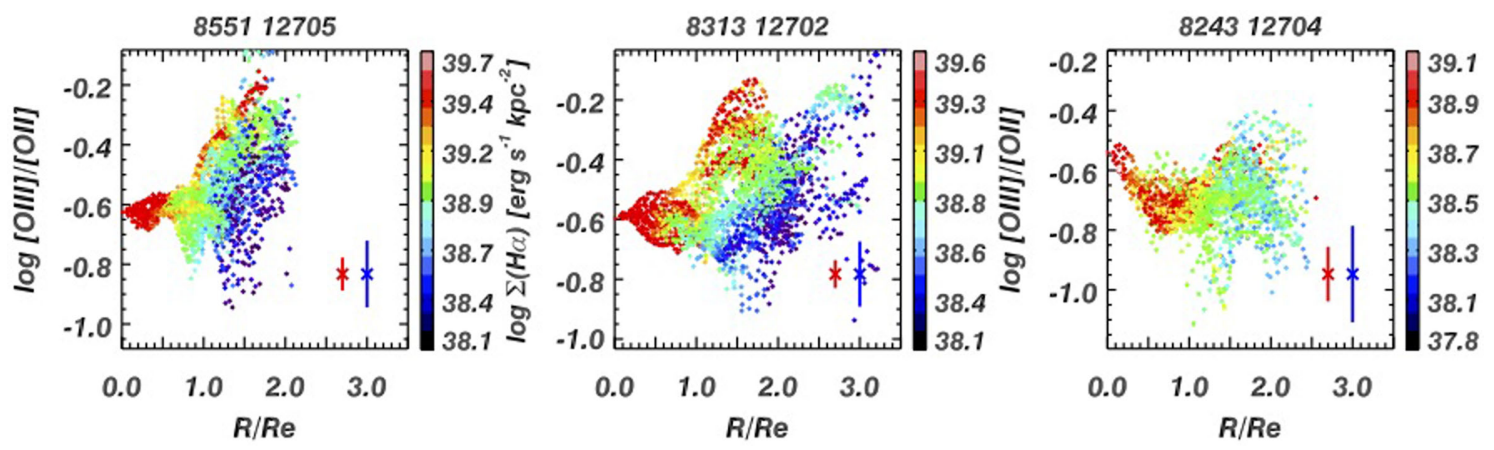

Figure 8. $\left[\mathrm{O}_{\mathrm{II}}\right] /\left[\mathrm{O}_{\mathrm{II}}\right]$ as a function of radius. The dots are colour-coded by $\Sigma_{\mathrm{H} \alpha}$. At a given radius, DIG shows lower $\left[\mathrm{O}_{\mathrm{III}}\right] /\left[\mathrm{O}_{\mathrm{II}}\right]$, meaning lower ionization parameter. The red and blue bars show the typical line ratio error at $\log \Sigma_{\mathrm{H} \alpha}=39$ and $38.5 \mathrm{erg} \mathrm{s}^{-1} \mathrm{kpc}^{-2}$ for individual spaxels. The length of the error bars is $2 \sigma$.
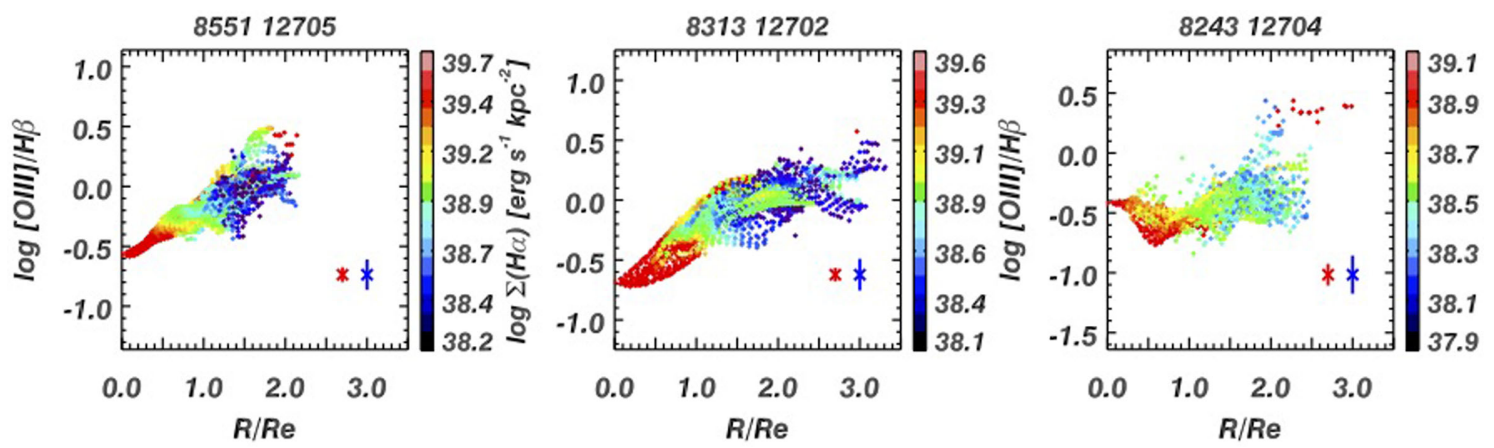

Figure 9. $\left[\mathrm{O}_{\mathrm{III}}\right] / \mathrm{H} \beta$ as a function of radius. The dots are colour-coded by $\Sigma_{\mathrm{H} \alpha}$. At a given radius, DIG might show higher or lower $[\mathrm{O} \mathrm{III}] / \mathrm{H} \beta$ than $\mathrm{H}_{\text {II }}$ regions depending on the specific situation of a galaxy. The red and blue bars show the typical line ratio error at $\log \Sigma_{\mathrm{H} \alpha}=39$ and $38.5 \mathrm{erg} \mathrm{s} \mathrm{s}^{-1} \mathrm{kpc}^{-2}$ for individual spaxels. The length of the error bars is $2 \sigma$.

shown that DIG has lower ionization parameter than $\mathrm{H}_{\text {II }}$ regions. At a fixed metallicity, a decrease in ionization parameter will result in a decrease of $\left[\mathrm{O}{ }_{\mathrm{III}}\right] / \mathrm{H} \beta$ for $q<7.5$ if no other parameter in the photoionization model is changed (see Fig. 15). For $q>7.5$, $[\mathrm{O}$ III $] / \mathrm{H} \beta$ is roughly constant and independent of ionization parameter. This diverse behaviour of $[\mathrm{O} \mathrm{III}] / \mathrm{H} \beta$ means that a third parameter other than metallicity and ionization parameter is needed, as will be discussed in Section 4. The third galaxy in Fig. 9, 8243-12704, shows a negative $[\mathrm{O} I I I] /[\mathrm{O} I \mathrm{II}]$ and $[\mathrm{O} \mathrm{III}] / \mathrm{H} \beta$ gradient. This is not consistent with an inverse metallicity gradient at the centre because the $[\mathrm{N} I I] /\left[\mathrm{O}_{\mathrm{II}}\right]$ gradient is negative in this range. This could be due to AGN activity, but the strength of the AGN is not strong enough to produce AGN-like line ratios on the BPT diagram. These kind of sources will be explored in future work.

\subsection{Line ratios versus $\Sigma_{\mathrm{H} \alpha}$ relation}

\subsubsection{Line ratios versus $\Sigma_{\mathrm{H} \alpha}$ relation: the whole sample}

To quantify the variation of the line ratios as a function of $\mathrm{H} \alpha$ surface brightness for the whole sample, we select all spaxels that have $\left[0.4 R_{\mathrm{e}}, 0.6 R_{\mathrm{e}}\right]$ in each galaxy. We then normalize the log line ratios versus $\log \Sigma_{\mathrm{H} \alpha}$ relation of each galaxy by subtracting from all the spaxels the median $\log$ line ratio and the median $\log \Sigma_{\mathrm{H} \alpha}$ of the set of spaxels. All the spaxels in a galaxy are weighted by $\frac{1}{N}$, where $N$ is the number of valid spaxels in this galaxy. Fig. 10 shows $\Delta \log$ line ratios versus $\Delta \log \Sigma_{\mathrm{H} \alpha}$ relation for the whole sample by combining all galaxies. $\left[\mathrm{S}_{\mathrm{II}}\right] / \mathrm{H} \alpha,\left[\mathrm{N}_{\mathrm{II}}\right] / \mathrm{H} \alpha,\left[\mathrm{O}_{\mathrm{II}}\right] / \mathrm{H} \beta,\left[\mathrm{O}_{\mathrm{I}}\right] / \mathrm{H} \alpha$ are higher in DIG-dominated low $\Sigma_{\mathrm{H} \alpha}$ regions. [O III]/[O II] is slightly lower in DIG while $[\mathrm{O} I I I] / \mathrm{H} \beta$ is mildly higher in DIG. 

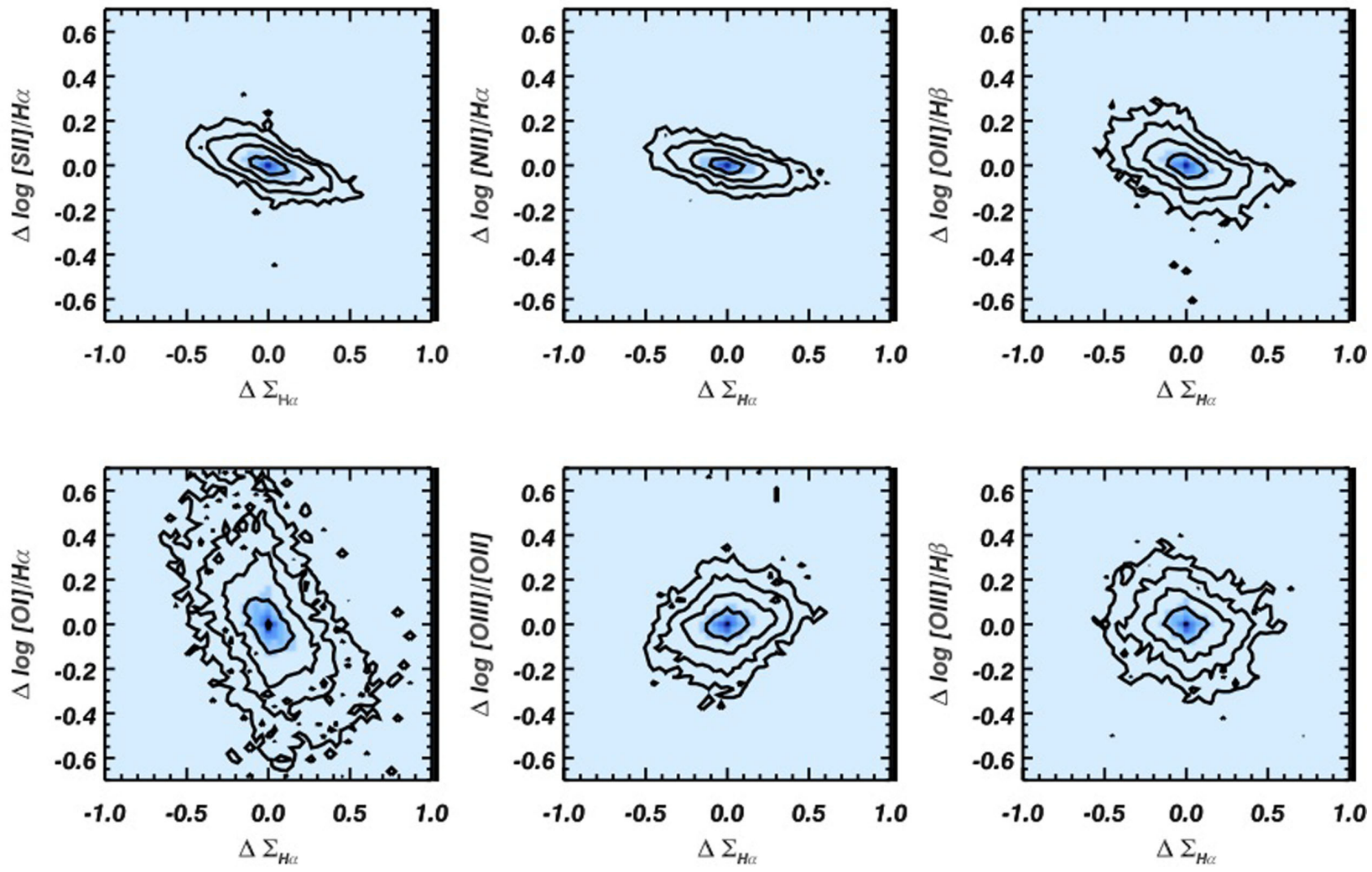

Figure 10. $\Delta \log$ (line ratios) versus $\Delta \log \Sigma_{\mathrm{H} \alpha}$ relation at $\left[0.4 R_{\mathrm{e}}, 0.6 R_{\mathrm{e}}\right]$ for all galaxies. We normalize the $\log \operatorname{line}$ ratios versus log $\Sigma_{\mathrm{H} \alpha}$ relation by subtracting the median $\log$ line ratio and median $\log \Sigma_{\mathrm{H} \alpha}$. All the spaxels in a galaxy are weighted by $\frac{1}{N}$, where $N$ is the number of valid spaxels in this galaxy. Contour levels are 5, 40, 70, 85 and 92 percentile. [S II]/H $\alpha,\left[\mathrm{N}\right.$ II]/H $\alpha,\left[\mathrm{O}_{\mathrm{II}}\right] / \mathrm{H} \beta,[\mathrm{O} \mathrm{I}] / \mathrm{H} \alpha$ are higher in DIG-dominated low $\Sigma_{\mathrm{H} \alpha}$ regions. [O III]/[O II] is slightly lower in DIG while $[\mathrm{O}$ III]/H $\beta$ is mildly higher in DIG.

Furthermore, we perform linear regression to the line ratio versus $\mathrm{H} \alpha$ surface brightness relation in narrow annuli for individual galaxies. For each galaxy, we get the slope of the linear regression at three radial bins: $\left[0.4 R_{\mathrm{e}}, 0.6 R_{\mathrm{e}}\right],\left[0.8 R_{\mathrm{e}}, 1.0 R_{\mathrm{e}}\right]$ and $\left[1.3 R_{\mathrm{e}}, 1.5 R_{\mathrm{e}}\right]$. The distribution of slopes for different line ratios at three radii are shown in black, blue, and red in Fig. 11. A negative slope means that the line ratio is enhanced in DIG, while a positive slope means the line ratio is lower in DIG. We see that the slope peaks around $\sim-0.3$ for $\log \left[\mathrm{S}_{\text {II }}\right] / \mathrm{H} \alpha, \log \left[\mathrm{N}_{\text {II }}\right] / \mathrm{H} \alpha$ and $\log \left[\mathrm{O}_{\text {II }}\right] / \mathrm{H} \beta$. The slopes for these three ratios are rarely positive. The slope peaks at -1.0 for $\log \left[\mathrm{O}_{\mathrm{I}}\right] / \mathrm{H} \alpha$, which is the most significant among the line ratios explored here. The slopes for $\left[\mathrm{O}_{\mathrm{III}}\right] /\left[\mathrm{O}_{\mathrm{II}}\right]$ are mostly positive, and its dispersion is larger than the dispersion for $\left[\mathrm{S}{ }_{\mathrm{II}}\right] / \mathrm{H} \alpha,[\mathrm{N} \mathrm{II}] / \mathrm{H} \alpha$ and $[\mathrm{O} I] / \mathrm{H} \beta$. The larger dispersion tells us that the ionization parameter varies significantly. The slope distribution for $\Delta \log [\mathrm{O} \mathrm{III}] / \mathrm{H} \beta$ versus $\Delta \log \Sigma_{\mathrm{H} \alpha}$ peaks at $\sim 0$, but the distribution is skewed to negative values. There are more galaxies with higher $[\mathrm{O} I I] / \mathrm{H} \beta$ in DIG than galaxies with lower $[\mathrm{O} \mathrm{III}] / \mathrm{H} \beta$ in DIG. The dispersion is even larger than that for $\left[\mathrm{O}_{\mathrm{III}}\right] /\left[\mathrm{O}_{\mathrm{II}}\right]$. This illustrates that $[\mathrm{O} \mathrm{III}] / \mathrm{H} \beta$ is very diverse in DIG. As shown in Section 3.3, DIG shows higher $\left[\mathrm{O}\right.$ III] $/ \mathrm{H} \beta$ than $\mathrm{H}_{\text {II }}$ regions in some galaxies, while it shows lower or similar $[\mathrm{O} \mathrm{III}] / \mathrm{H} \beta$ for other galaxies. Fig. 11 demonstrates that DIG with higher $\left[\mathrm{S}_{\mathrm{II}}\right] / \mathrm{H} \alpha,\left[\mathrm{N}_{\mathrm{II}}\right] / \mathrm{H} \alpha,\left[\mathrm{O}_{\mathrm{II}}\right] / \mathrm{H} \beta,\left[\mathrm{O}_{\mathrm{I}}\right] / \mathrm{H} \alpha$ and lower $[\mathrm{O} \mathrm{III}] /[\mathrm{O} I \mathrm{II}]$ than $\mathrm{H}$ II regions is prevalent among all galaxies in our sample. $\left[\mathrm{O}_{\mathrm{III}}\right] / \mathrm{H} \beta$ could be significantly higher or lower than $\mathrm{H}_{\mathrm{II}}$ regions. The contamination of DIG influences every star-forming galaxy.

\subsubsection{Line ratios versus $\Sigma_{\mathrm{H} \alpha}$ relation: split by stellar mass}

In Figs 12 and 13, we show the $\Delta \log$ line ratio versus $\Delta \log$ $\Sigma_{\mathrm{H} \alpha}$ relations for galaxies with stellar mass less than $10^{9.43}$ and higher than $10^{10.08}$, respectively. These are the one third least massive and the one third most massive galaxies in our sample. The slopes of $\Delta \log \left[\mathrm{S}_{\mathrm{II}}\right] / \mathrm{H} \alpha,\left[\mathrm{N}_{\mathrm{II}}\right] / \mathrm{H} \alpha,\left[\mathrm{O}_{\mathrm{II}}\right] / \mathrm{H} \beta,\left[\mathrm{O}_{\mathrm{I}}\right] / \mathrm{H} \alpha$ and $\left[\mathrm{O}_{\mathrm{III}}\right] /\left[\mathrm{O}_{\mathrm{II}}\right]$ versus $\Delta \log \Sigma_{\mathrm{H} \alpha}$ relations do not depend much on stellar mass. However, massive galaxies show a significantly negative $\Delta \log [\mathrm{O} \mathrm{III}] / \mathrm{H} \beta$ versus $\Delta \log \Sigma_{\mathrm{H} \alpha}$ relation, while less massive galaxies show a positive $\Delta \log \left[\mathrm{O}_{\mathrm{III}}\right] / \mathrm{H} \beta$ versus $\Delta \log \Sigma_{\mathrm{H} \alpha}$ relation. In other words, $[\mathrm{O}$ III $] / \mathrm{H} \beta$ of DIG is always enhanced relative to $\mathrm{H}_{\text {II }}$ regions in the most massive galaxies. A leaky $\mathrm{H}_{\text {II }}$ region model cannot produce high $\left[\mathrm{O}\right.$ III] $/ \mathrm{H} \beta$ relative to $\mathrm{H}_{\mathrm{II}}$ regions $(\mathrm{Sec}$ tion 4.2). The dependence of the $\Delta \log \left[\mathrm{O}_{\mathrm{III}}\right] / \mathrm{H} \beta$ versus $\Delta \log \Sigma_{\mathrm{H} \alpha}$ relation on stellar mass may indicate different ionization mechanisms in galaxies of different masses. We leave the study of the physical reason behind the dependence of $\Delta$ log line ratios versus $\Delta \log \Sigma_{\mathrm{H} \alpha}$ relation on stellar mass for future studies.

\section{DIAGNOSTIC DIAGRAMS}

\subsection{Comparison of line ratios with $\mathrm{H}$ II region grids}

In Fig. 14, we plot the line ratios for each spaxel on the [O III]/[O II] versus $\left[\mathrm{N}_{\mathrm{II}}\right] /\left[\mathrm{O}_{\mathrm{II}}\right]$ diagram (Dopita et al. 2000). [ $\left.\mathrm{N}_{\mathrm{II}}\right] /\left[\mathrm{O}_{\mathrm{II}}\right]$ is sensitive to metallicity because $N$ is a secondary element. At high 

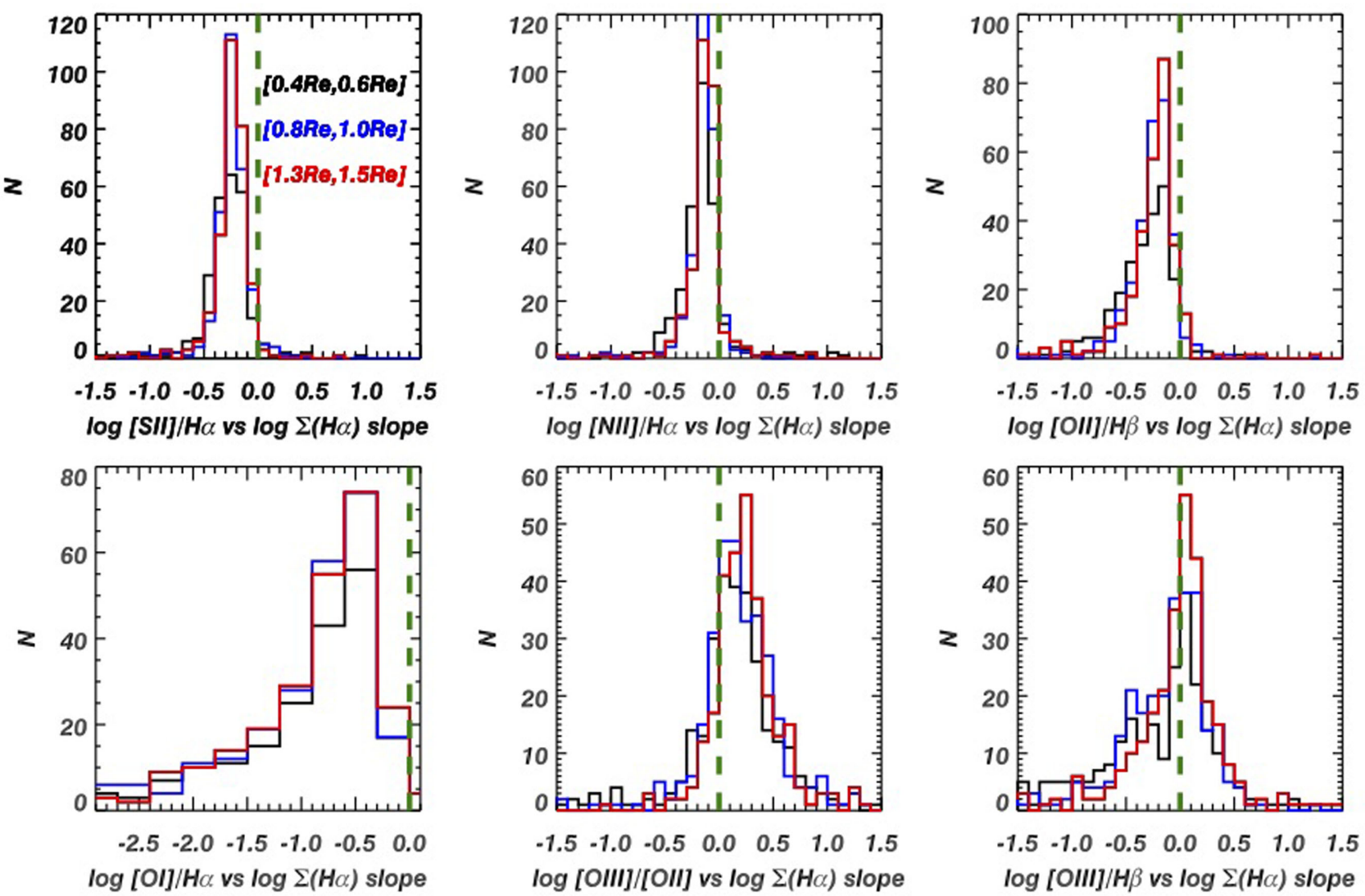

Figure 11. The distribution of $\Delta \log$ line ratios versus $\Delta \log \Sigma_{\mathrm{H} \alpha}$ slope at three radii. The black line is for the radius at $\left[0.4 R_{\mathrm{e}}, 0.6 R_{\mathrm{e}}\right]$, the blue line is for the radius at $\left[0.8 R_{\mathrm{e}}, 1.0 R_{\mathrm{e}}\right]$ and the red line is for the radius at $\left[1.3 R_{\mathrm{e}}, 1.5 R_{\mathrm{e}}\right]$. The green dashed line is the slope $=0$ line for reference. For almost all star-forming galaxies in our sample, DIG shows higher $\left.\left[\mathrm{S}_{\mathrm{II}}\right] / \mathrm{H} \alpha\left[\mathrm{N}_{\mathrm{II}}\right] / \mathrm{H} \alpha\left[\mathrm{O}_{\mathrm{II}}\right] / \mathrm{H} \beta,{ }_{[\mathrm{O}}\right] / \mathrm{H} \alpha$ and lower $\left[\mathrm{O}_{\mathrm{III}}\right] /\left[\mathrm{O}\right.$ II] than $\mathrm{H}_{\mathrm{II}}$ regions. [O III] $] / \mathrm{H} \beta$ in $\mathrm{DIG}$ can be higher or lower than in $\mathrm{H}$ II regions.

metallicity, $\left[\mathrm{O}_{\mathrm{II}}\right]$ is suppressed due to the decrease in temperature. $\left[\mathrm{O}_{\mathrm{III}}\right] /\left[\mathrm{O}_{\mathrm{II}}\right]$ is a very good proxy of the ionization parameter. We overplot the most up-to-date grids for $\mathrm{H}_{\text {II }}$ regions from Dopita et al. (2013) with $\kappa=I n f$, which means that the electrons obey the Maxwell-Boltzmann distribution. Our data fall within the grids. However, when we turn to the BPT diagrams in Figs 15 and 16 , we see that while the high $\mathrm{H} \alpha$ surface brightness spaxels are still consistent with the grid prediction, DIG-dominated low-H $\alpha$ surface brightness regions are located outside the model grids. Specifically, the $\left[\mathrm{N}_{\mathrm{II}}\right] / \mathrm{H} \alpha$ and $\left[\mathrm{S}_{\mathrm{II}}\right] / \mathrm{H} \alpha$ are significantly enhanced in DIG. Previous work found a similar phenomenon for DIG (e.g. Galarza et al. 1999; Hoopes \& Walterbos 2003; Kaplan et al. 2016). This means that $\mathrm{H}$ II region models with only a lower $q$ cannot explain the low-ionization line ratios like $\left[\mathrm{S}_{\mathrm{II}}\right] / \mathrm{H} \alpha$ and $\left[\mathrm{N}_{\mathrm{II}}\right] / \mathrm{H} \alpha$. Apart from metallicity and ionization parameter, there must be other parameter(s) that govern the behaviour of the emission line.

The location of DIG on the BPT diagrams gives us some clue to what the other parameters could be. DIG enters the composite or $\mathrm{LI}(\mathrm{N}) \mathrm{ER}$ regions on the $\left[\mathrm{O}{ }_{\mathrm{III}}\right] / \mathrm{H} \beta$ versus $\left[\mathrm{N}{ }_{\mathrm{II}}\right] / \mathrm{H} \alpha$ and $[\mathrm{O} I I I] / H \beta$ versus $\left[\mathrm{S}_{\mathrm{II}}\right] / \mathrm{H} \alpha$ diagrams. AGN and $\mathrm{LI}(\mathrm{N}) \mathrm{ER}$ are characterized by their hard ionizing spectrum. A hard spectrum is capable of producing a large partially ionized region that enhances the $\left[\mathrm{S}_{\mathrm{II}}\right] / \mathrm{H} \alpha$ and $\left[\mathrm{N}{ }_{\mathrm{II}}\right] / \mathrm{H} \alpha$, just like what we observe. Thus, a harder ionizing spectrum is one possible answer to the line ratios enhancement.

\subsection{Photoionization grid}

To get a quantitative idea of how hardness can change line ratios, we use CLOUDY (Ferland et al. 1998) to calculate new grids for a series of incident spectra. We test two models that are capable of producing a hard ionizing spectrum: a leaky $\mathrm{H}$ II region model and a hot evolved star model. The leaky $\mathrm{H}$ II region model can have a harder ionizing spectrum because the lower energy part is more likely to be absorbed by the IGM (e.g. Giammanco, Beckman \& Cedrés 2005). Hot evolved stars, like pAGB stars, are characterized by very high temperatures, yielding a hard ionizing spectrum. The input spectra to test the two models include (see Fig. 17):

(1) The spectrum of an O star with $T_{\text {eff }}=42300 \mathrm{~K}, \log g=$ 4.22, solar metallicity. (Tlusty OSTAR2002; Lanz \& Hubeny 2003; magenta line)

(2) Spectrum in (1) filtered through a gas cloud with $n=100 \mathrm{~cm}^{-3}$ and $N_{\mathrm{H}}=10^{18} \mathrm{~cm}^{-2}$. The resulting spectrum includes both the transmitted spectrum and the emission from the cloud itself (orange line).

(3) Spectrum in (1) filtered through a gas cloud with $n_{\mathrm{e}}=100 \mathrm{~cm}^{-3}$ and $N_{\mathrm{H}}=10^{18.5} \mathrm{~cm}^{-2}$ (green line).

(4) Spectrum in (1) filtered through a gas cloud with $n_{\mathrm{e}}=100 \mathrm{~cm}^{-3}$ and $N_{\mathrm{H}}=10^{18.7} \mathrm{~cm}^{-2}$ (cyan line).

(5) Spectrum in (1) filtered through a gas cloud with $n_{e}=100 \mathrm{~cm}^{-3}$ and $N_{H}=10^{18.9} \mathrm{~cm}^{-2}$ (blue line). 

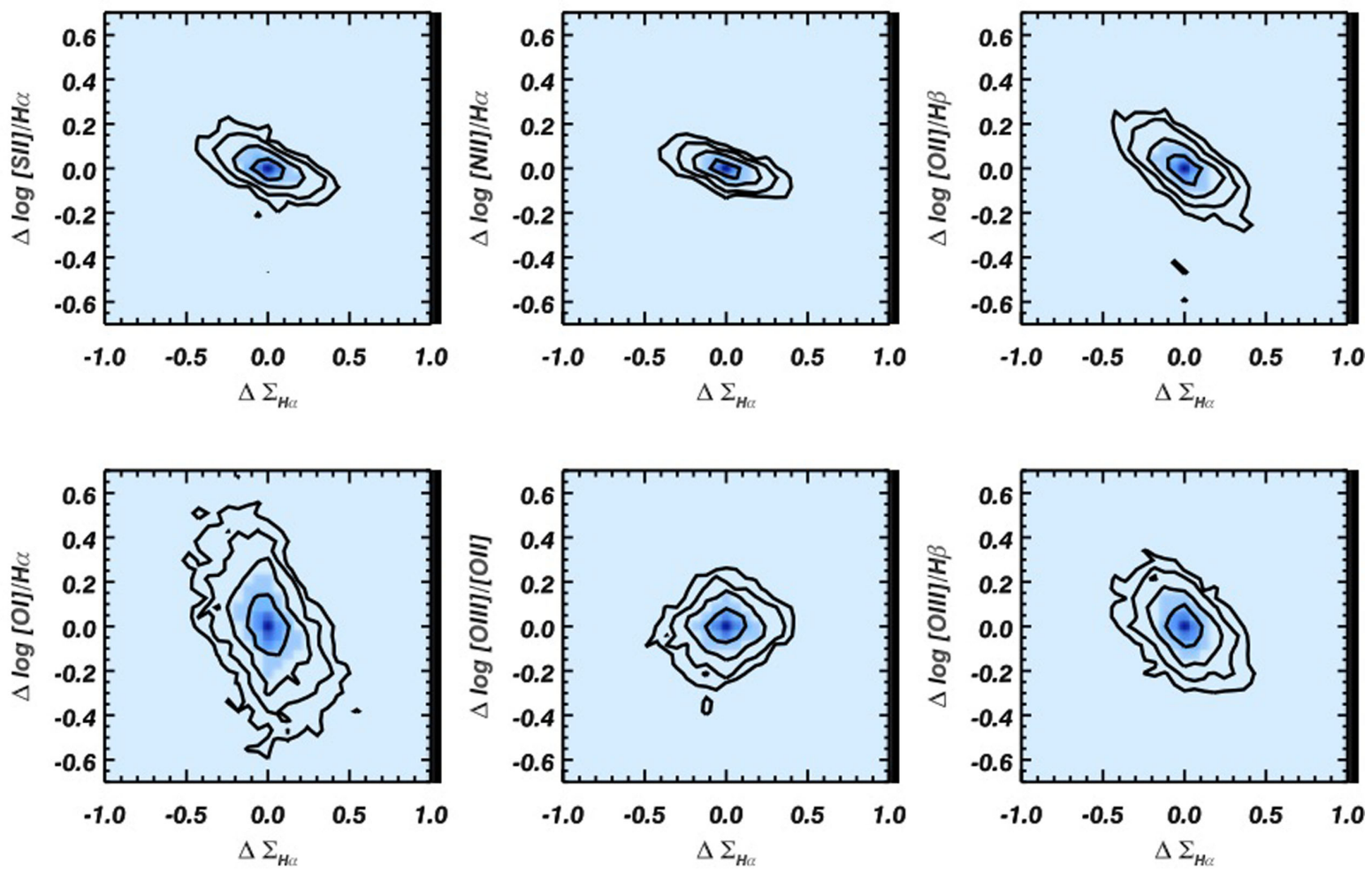

Figure 12. The $\Delta \log$ (line ratios) versus $\Delta \log \Sigma_{\mathrm{H} \alpha}$ relation at $\left[0.4 R_{\mathrm{e}}, 0.6 R_{\mathrm{e}}\right]$ for the one-third most massive galaxies. We normalize the log line ratios versus $\log \Sigma_{\mathrm{H} \alpha}$ relation by subtracting the median $\log$ line ratio and median $\log \Sigma_{\mathrm{H} \alpha}$. All the spaxels in a galaxy are weighted by $\frac{1}{N}$, where $N$ is the number of

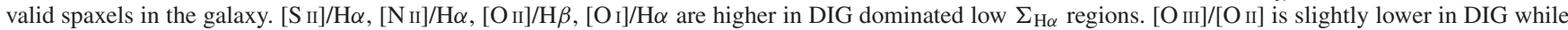
$[\mathrm{O} \mathrm{III}] / \mathrm{H} \beta$ is significantly higher in DIG. $[\mathrm{O} \mathrm{III}] / \mathrm{H} \beta$ in DIG of massive galaxies is different from that in less massive galaxies.

(6) The spectrum of a 13 Gyr SSP generated with BC03 (red line).

The metallicity of the ionized cloud is that of Orion nebula (Baldwin et al. 1991; Rubin et al. 1991; Osterbrock, Tran \& Veilleux 1992; Rubin, Dufour \& Walter 1993). The density of the cloud for model (1) is $10 \mathrm{~cm}^{-3}$, and $1 \mathrm{~cm}^{-3}$ for models (2)-(6). We run the calculation for $\log U=-4.5$ to -2.0 with an interval of 0.5 $\operatorname{dex}(\log U=\log q / c, c$ is the speed of light).

Model (1) represents a typical $\mathrm{H}_{\text {II }}$ region. Models (2)-(5) are for testing the leaky $\mathrm{H}_{\text {II }}$ region model. The hardening of the ionizing spectrum is obvious when compared with (1). Model (6) simulates LI(N)ER-like ionization by an old stellar population that has been proposed to explain the emission seen in a large fraction of passive galaxies (e.g. Sarzi et al 2006; Stasińska et al. 2008; Kehrig et al. 2012; Yan \& Blanton 2012; Singh et al. 2013; Belfiore et al. 2016a,b; Gomes et al. 2016). Evolved stellar populations have a very hard spectrum. In Fig. 18, we show the diagnostic diagrams for the different incident spectra listed above. The O star model lies near the Kauffmann demarcation on the $[\mathrm{O}$ III] $/ \mathrm{H} \beta$ versus $[\mathrm{N} \mathrm{II]} / \mathrm{H} \alpha$ and $\left[\mathrm{O}{ }_{I I I}\right] / \mathrm{H} \beta$ versus $\left[\mathrm{S}_{\mathrm{II}}\right] / \mathrm{H} \alpha$ diagrams as expected. The $13 \mathrm{Gyr}$ SSP grid shows significant higher $\left[\mathrm{S}_{\mathrm{II}}\right] / \mathrm{H} \alpha,\left[\mathrm{N}_{\mathrm{II}}\right] / \mathrm{H} \alpha$ and $\left[\mathrm{O}_{\mathrm{II}}\right] / \mathrm{H} \alpha$ relative to $\mathrm{H}_{\text {II }}$ regions with the same ionization parameters due to a more extended partially ionized region. Models (2) and (3) show a harder ionizing spectrum than $\mathrm{H}_{\mathrm{II}}$ region, but their line ratios are similar to those for $\mathrm{H}_{\text {II }}$ regions. Model (4) exhibits enhancement in $[\mathrm{N} I I] / \mathrm{H} \alpha,\left[\mathrm{S}_{\mathrm{II}}\right] / \mathrm{H} \alpha$ and $\left[\mathrm{O}{ }_{\mathrm{II}}\right] / \mathrm{H} \alpha$ relative to $\mathrm{H}$ II regions. The enhancements are small, typically less than 0.2 dex. However, model (5), which has the hardest spectrum blueward of $912 \AA$ shows the lowest $\left[\mathrm{N}_{I I}\right] / \mathrm{H} \alpha,\left[\mathrm{S}_{I I}\right] / \mathrm{H} \alpha$ and $\left[\mathrm{O}_{\Pi}\right] / \mathrm{H} \alpha$. This is because even though those photons lower than $13.6 \mathrm{eV}$ cannot ionize hydrogen, they have enough energy to excite an electron in the hydrogen atom from ground level $(n=1)$ to $n=3$ or 4 level. When they jump back, they can jump to $n=2$ that will produce $\mathrm{H} \alpha$ and $\mathrm{H} \beta$ lines. This is fluorescent production. In model (5), photons pass through a very high column density that absorbs more than 99.9 per cent of all the ionizing photons. When the transmitted spectrum is forced to have $\log U>-4.5$, we obtain an unphysically high continuum redward of $912 \AA$, which leads to large fluorescent production. It is the enhancement of $\mathrm{H} \alpha$ due to fluorescent production that gives rise to lower $\left[\mathrm{N}\right.$ II] $/ \mathrm{H} \alpha,\left[\mathrm{S}_{\mathrm{II}}\right] / \mathrm{H} \alpha,\left[\mathrm{O}_{\mathrm{II}}\right] / \mathrm{H} \alpha$ and $\left[\mathrm{O}_{\mathrm{I}}\right] / \mathrm{H} \alpha$.

Considering the large amount of ionizing photons from OB stars and the spatial correlation of DIG and $\mathrm{H}$ II regions, leaky $\mathrm{H}$ II regions are likely a major mechanism for producing DIG. However, our results disfavour leaky $\mathrm{H}$ II region models to account for the ionization of all DIG. DIG has a lower ionization parameter than $\mathrm{H}$ II regions, a decrease in ionization parameter leads to enhancement of $[\mathrm{N}$ $\left[\mathrm{S}_{\mathrm{II}}\right] / \mathrm{H} \alpha,\left[\mathrm{O}_{\text {II }}\right] / \mathrm{H} \alpha$ and $\left[\mathrm{O}_{\mathrm{I}}\right] / \mathrm{H} \alpha$, but a decrease in $\left[\mathrm{O}_{\mathrm{III}}\right] / \mathrm{H} \beta$ at the same time. Leaky $\mathrm{H}_{\text {II }}$ region models cannot produce $\mathrm{LI}(\mathrm{N}) \mathrm{ER}$-like emission. The cyan line shows enhancement of these four line ratios, but it needs fine tuning of the filtering column density. DIG that shows LI(N)ER-like emission needs another ionization source. We favour evolved stars as a major ionization source for DIG because only ionization by evolved stars (red line, Model 6) can produce enhancement of $\left[\mathrm{S}_{\mathrm{II}}\right] / \mathrm{H} \alpha,[\mathrm{N}$ II] $] / \mathrm{H} \alpha,\left[\mathrm{O}_{\text {II }}\right] / \mathrm{H} \alpha,\left[\mathrm{O}_{\mathrm{I}}\right] / \mathrm{H} \alpha$ and $[\mathrm{O}$ III] $] / \mathrm{H} \beta$ even when the ionization parameter drops. Evolved stars are prevalent all over galaxies and their contribution to ionizing photons may prevail. Flores-Fajardo et al. (2011) proposed hot low-mass evolved stars (HOLMES) as an important ionization source for the extraplanar DIG in edge-on galaxies. For NGC 891, HOLMES begin to 

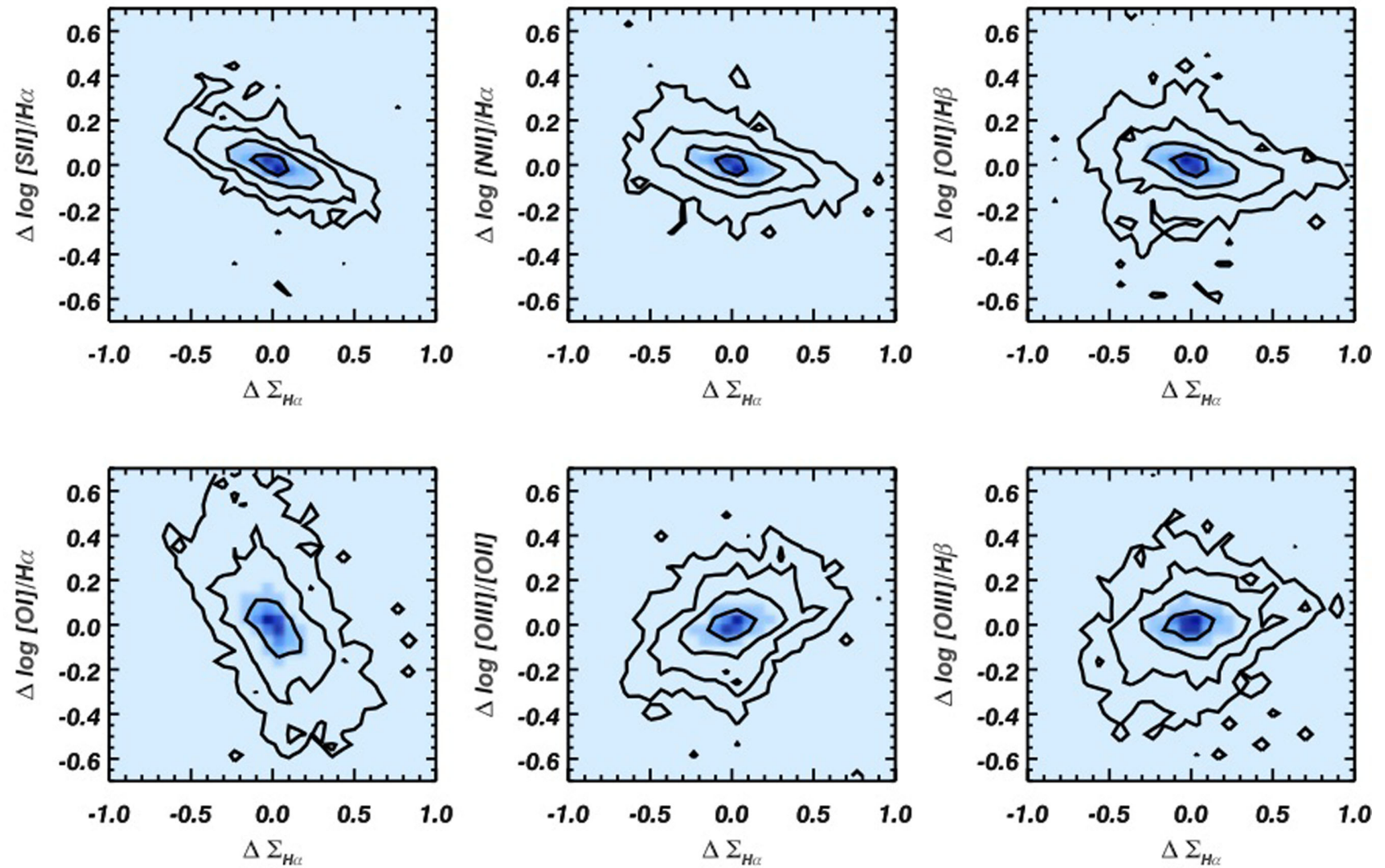

Figure 13. The $\Delta \log$ (line ratios) versus $\Delta \log \Sigma_{\mathrm{H} \alpha}$ relation at $\left[0.4 R_{\mathrm{e}}, 0.6 R_{\mathrm{e}}\right]$ for the one-third least massive galaxies. We normalize the log line ratios versus $\log \Sigma_{\mathrm{H} \alpha}$ relation by subtracting the median $\log$ line ratio and median $\log \Sigma_{\mathrm{H} \alpha}$. All the spaxels in a galaxy are weighted by $\frac{1}{N}$, where $N$ is the number of valid spaxels in the galaxy. $\left[\mathrm{S}_{\mathrm{II}}\right] / \mathrm{H} \alpha,\left[\mathrm{N}\right.$ II] $/ \mathrm{H} \alpha,\left[\mathrm{O}\right.$ II] $/ \mathrm{H} \beta,\left[\mathrm{O}_{\mathrm{I}}\right] / \mathrm{H} \alpha$ are higher in DIG-dominated low $\Sigma_{\mathrm{H} \alpha}$ regions. $\left[\mathrm{O}\right.$ III] $/\left[\mathrm{O}_{\mathrm{II}}\right]$ is slightly lower in DIG while $[\mathrm{O}$ III] $] / \mathrm{H} \beta$ is slightly lower in DIG.
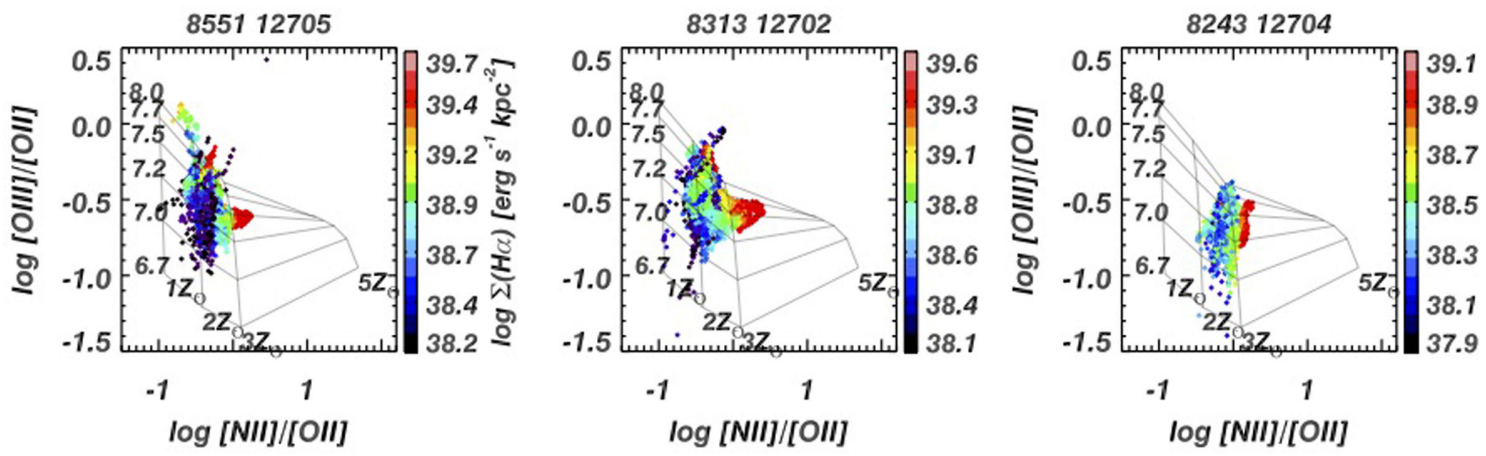

Figure 14. [ $\left.\mathrm{O}_{\mathrm{III}}\right] /\left[\mathrm{O}_{\mathrm{II}}\right]$ versus $\left[\mathrm{N}_{\mathrm{II}}\right] /\left[\mathrm{O}_{\mathrm{II}}\right]$ diagram (Dopita et al. 2000, 2013). The grids are from Dopita et al. (2013) with $\kappa=$ Inf. The nearly horizontal lines are for constant ionization parameters and the nearly vertical lines are for constant metallicities. The labels denote the metallicities and ionization parameters $(\log q)$ for the grid.

contribute more than 50 per cent of the ionizing photons at scaleheight $|z|>2 \mathrm{kpc}$. We propose here that the evolved stars are not only capable of ionizing the extraplanar gas, but also an important ionizing source for the DIG near the disc.

\subsection{Photoionization grids for SSP at different ages}

We have shown in last subsection that a 13 Gyr SSP can produce the LI(N)ER-like emission we see for DIG. One interesting question is: How would the line ratios change as an SSP ages? To answer this question, we generate SSPs at $1 \mathrm{Myr}, 3 \mathrm{Myr}, 9 \mathrm{Myr}, 0.125 \mathrm{Gyr}$,
7 Gyr and 13 Gyr using BC03, and use these spectra as the incident ionizing spectra for CLOUDY. The spectra from 0.125 to $13 \mathrm{Gyr}$ change only slightly. The metallicity is that of the Orion nebula, and the density is $1 \mathrm{~cm}^{-3}$. We run the calculation for $\log U=-4.5$ to -2.0 with an interval of $0.5 \mathrm{dex}$. The incident spectra are shown in Fig. 19. The output line ratios are plotted in the same colour as their incident spectra in Fig. 20. At 1 Myr, the line ratios are located at the $\mathrm{H}$ II region part on the diagnostic diagrams. At $3 \mathrm{Myr}$, the line ratios are similar to those of $1 \mathrm{Myr}$. At $9 \mathrm{Myr}$, however, we see a significant decrease in $[\mathrm{N}$ II] $] / \mathrm{H} \alpha,\left[\mathrm{S}_{\mathrm{II}}\right] / \mathrm{H} \alpha,\left[\mathrm{O}_{\mathrm{II}}\right] / \mathrm{H} \alpha,\left[\mathrm{O}_{\mathrm{III}}\right] / \mathrm{H} \beta,[\mathrm{O}$ III]/[O II] and an increase in $[\mathrm{N}$ II $] /\left[\mathrm{O}_{\text {II }}\right]$. This is due to the significant decrease 

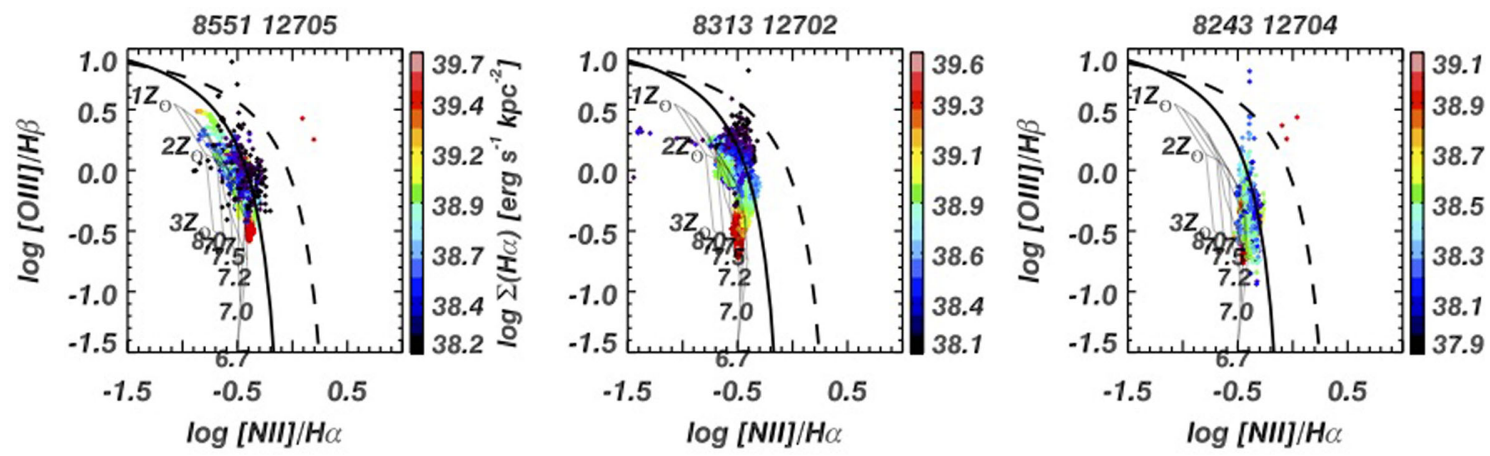

Figure 15. $[\mathrm{O}$ III] $] / \mathrm{H} \beta$ versus $[\mathrm{N}$ II $] / \mathrm{H} \alpha$ diagram for each galaxy. Different colours represent different $\Sigma_{\mathrm{H} \alpha}$. The solid and dashed lines are the demarcation line from Kauffmann et al. (2003) and Kewley et al. (2001). The grids are from Dopita et al. (2013) with $\kappa=$ Inf. DIG cannot be covered by the grids, suggesting $\mathrm{H}$ II region models with variations of metallicity and ionization parameter cannot produce the DIG line ratios.
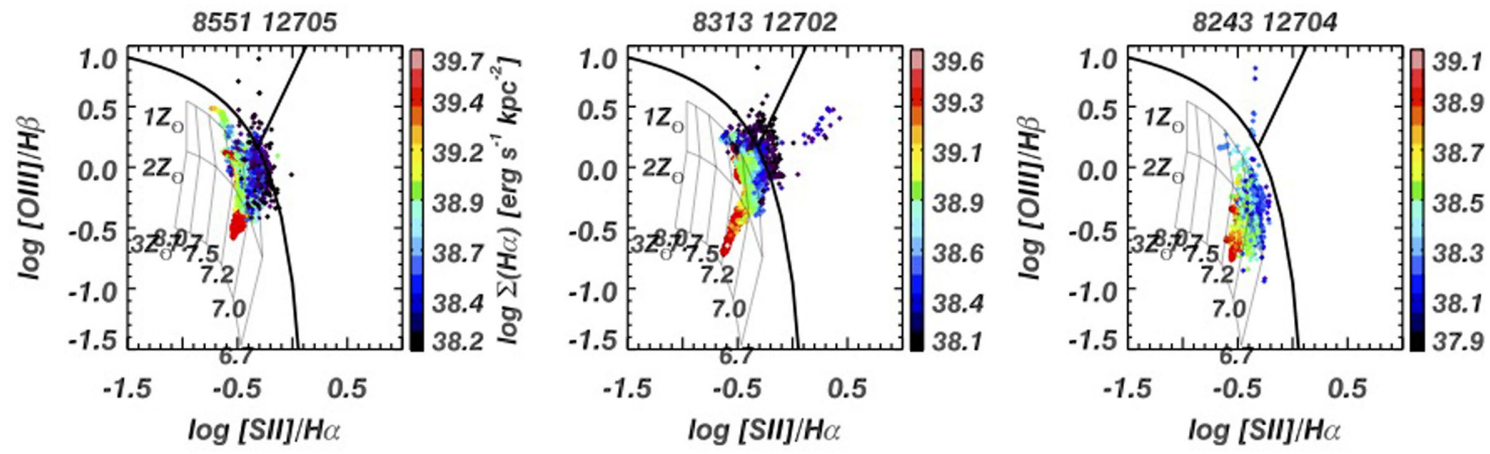

Figure 16. $\left[\mathrm{O}_{\mathrm{III}}\right] / \mathrm{H} \beta$ versus $\left[\mathrm{S}_{\mathrm{II}}\right] / \mathrm{H} \alpha$ diagram for each galaxy. Different colours represent different $\Sigma_{\mathrm{H} \alpha}$. The solid lines are the demarcation lines to separate Seyfert galaxies from LINER in Kewley et al. (2006). The grids are from Dopita et al. (2013) with $\kappa=$ Inf. The grids do not cover DIG, suggesting that H II region models with variations of metallicity and ionization parameter cannot produce the DIG line ratios.

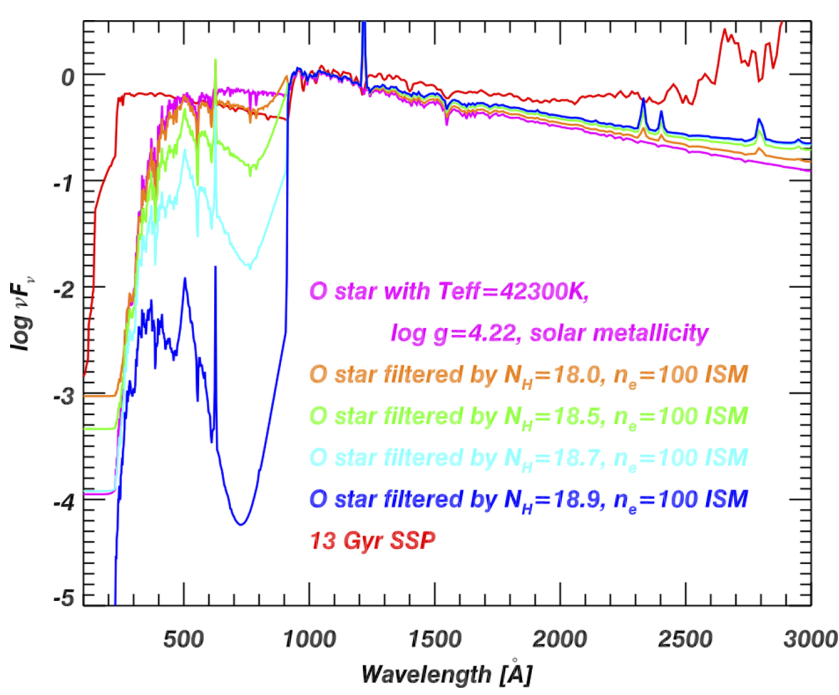

Figure 17. The incident spectra for our models described in Section 4.2. All spectra are normalized to the flux at $1000 \AA$. The magenta line is a $42300 \mathrm{~K}, \log g=4.22$, solar metallicity O star generated by Tlusty (Lanz \& Hubeny 2003). The orange, green, cyan and blue lines are the spectra by filtering the $\mathrm{O}$ star spectrum through column density of $10^{18} \mathrm{~cm}^{-1}$, $10^{18.5} \mathrm{~cm}^{-1}, 10^{18.7} \mathrm{~cm}^{-1}, 10^{18.9} \mathrm{~cm}^{-1}$, simulating the leaky $\mathrm{H}_{\text {II }}$ regions. The red line is the spectrum of a 13 Gyr SSP generated by BC03. in 200-500 Å spectrum hardness as OB stars age (Levesque, Kewley \& Larson 2010). At $125 \mathrm{Myr}$, the line ratios are already in the $\mathrm{LI}(\mathrm{N}) \mathrm{ER} / \mathrm{AGN}$ region. This means that once OB stars die, the line ratios of the ionized gas would turn into ' $\mathrm{LI}(\mathrm{N}) \mathrm{ER} / \mathrm{AGN}$-like' very fast. The line ratios do not change very much afterwards. This sheds light on the interpretation of LI(N)ER/AGN like emissionline ratios in galaxies. When $\mathrm{OB}$ stars are alive, their light dominates the ionizing spectrum and the ionized gas located in the star-forming regions dominates on the diagnostic diagrams. When OB stars cease to form, their contribution disappears very fast and the light of hot evolved stars begins to dominate after tens of Myr, and the ionized gas is located in the $\mathrm{LI}(\mathrm{N}) \mathrm{ER} / \mathrm{AGN}$ regions on the diagnostic diagrams. We have shown in Section 4.2 that a filtered spectrum cannot reproduce the DIG line ratios with an increase of $[\mathrm{O} \mathrm{III}] / \mathrm{H} \beta$. A higher $\left[\mathrm{O}_{\mathrm{III}}\right] / \mathrm{H} \beta$ in DIG than in $\mathrm{H}_{\mathrm{II}}$ regions means that the ionization of DIG may not be linked to OB stars directly but linked to the hot evolved stars.

We are not claiming that evolved stellar populations are the major ionizing source of DIG. Overall, the radiative and mechanical energy from hot evolved stars falls short of the energy budget of DIG in star-forming galaxies (Reynolds 1984; Binette et al. 1994; Ferguson et al. 1996). Even if the energy emitted by hot evolved stars meets the energy requirement of DIG, O stars can provide at least one order of magnitude more ionizing photons than hot evolved stars in starforming galaxies (Reynolds 1984; Ferguson et al. 1996). Indeed, DIG is found to be linked to $\mathrm{H}$ II regions both along the disc and in 

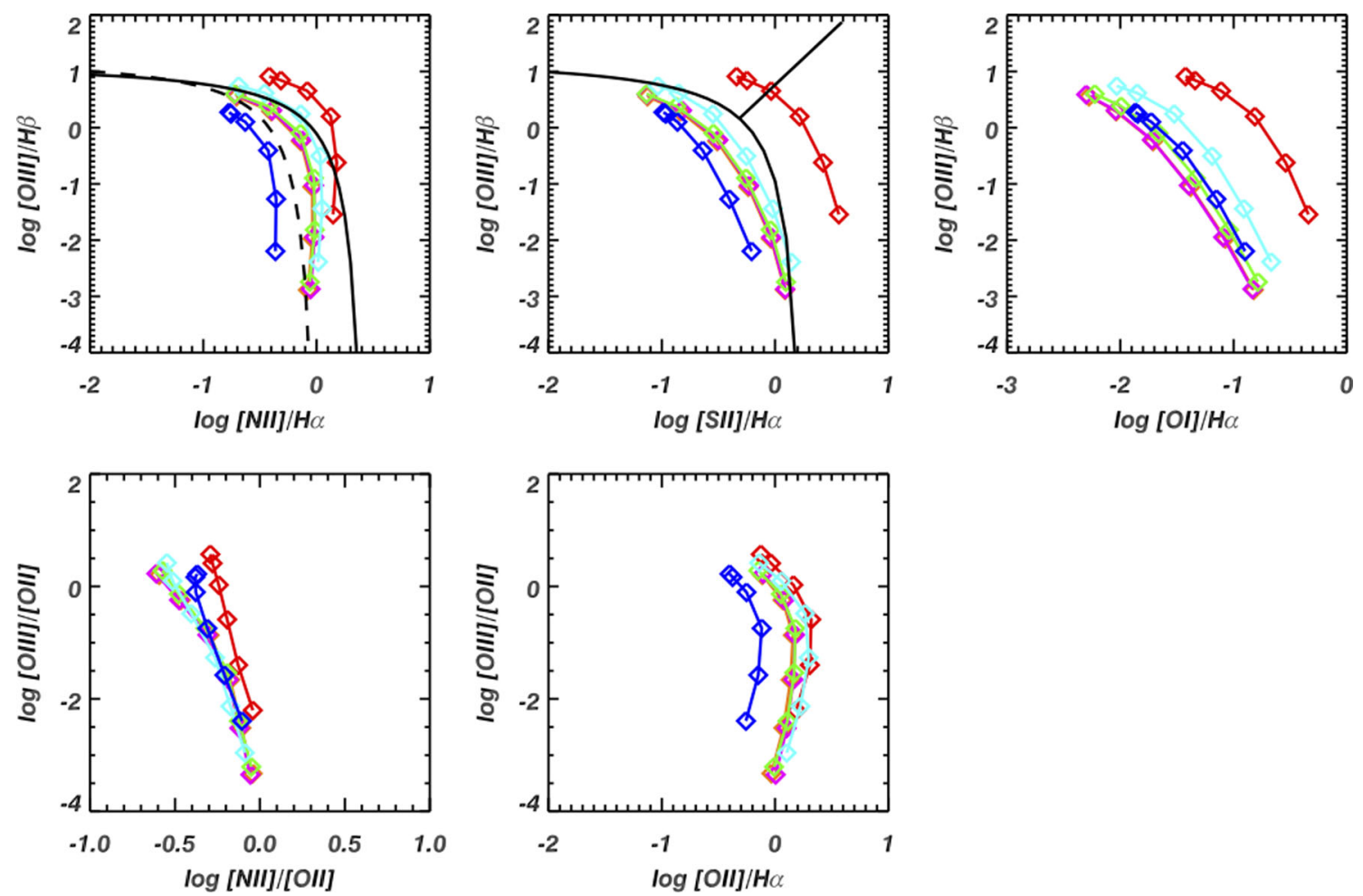

Figure 18. The BPT diagrams, $\log \left[\mathrm{O}_{\mathrm{III}}\right] / \mathrm{H} \beta$ versus $\log \left[\mathrm{O}_{\mathrm{I}}\right] / \mathrm{H} \alpha$ diagram, $\log \left[\mathrm{O}_{\mathrm{III}}\right] /\left[\mathrm{O}_{\mathrm{II}}\right]$ versus $\log \left[\mathrm{N}_{\mathrm{II}}\right] /\left[\mathrm{O}_{\mathrm{II}}\right]$ diagram, and $\log \left[\mathrm{O}_{\mathrm{III}}\right] /\left[\mathrm{O}_{\mathrm{II}}\right]$ versus $\log \left[\mathrm{O}_{\mathrm{II}}\right] / \mathrm{H} \alpha$ diagrams for different incident spectra shown in Fig. 17. The colour scheme is the same as in Fig. 17. The dots with the same colour have different ionization parameters from $\log U=-4.5$ to $\log U=-2$ with an interval of 0.5 dex. The leaky $\mathrm{H}$ II region models (orange, green, cyan lines) show very similar line ratios to the $\mathrm{O}$ star model (magenta line) at the same ionization parameter. We note that DIG has lower ionization parameters than HII regions.

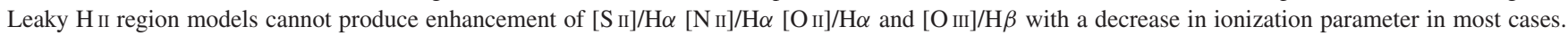
The cyan line shows enhancement of these three line ratios, but it needs fine tuning of the filtering column density. The blue line even shows decreases in $[\mathrm{S} I] / \mathrm{H} \alpha[\mathrm{N}$ II] $/ \mathrm{H} \alpha[\mathrm{O} I \mathrm{II}] / \mathrm{H} \alpha$ and $[\mathrm{O} \mathrm{III}] / \mathrm{H} \beta$, because of large $\mathrm{H} \alpha$ florescent production, which is unphysical. Only ionization by evolved stellar populations (red line) can produce enhancement of $\left[\mathrm{S}\right.$ II] $/ \mathrm{H} \alpha\left[\mathrm{N}\right.$ II] $/ \mathrm{H} \alpha\left[\mathrm{O}_{\mathrm{II}}\right] / \mathrm{H} \alpha$ and $[\mathrm{O} \mathrm{III}] / \mathrm{H} \beta$ even when the ionization parameter drops.

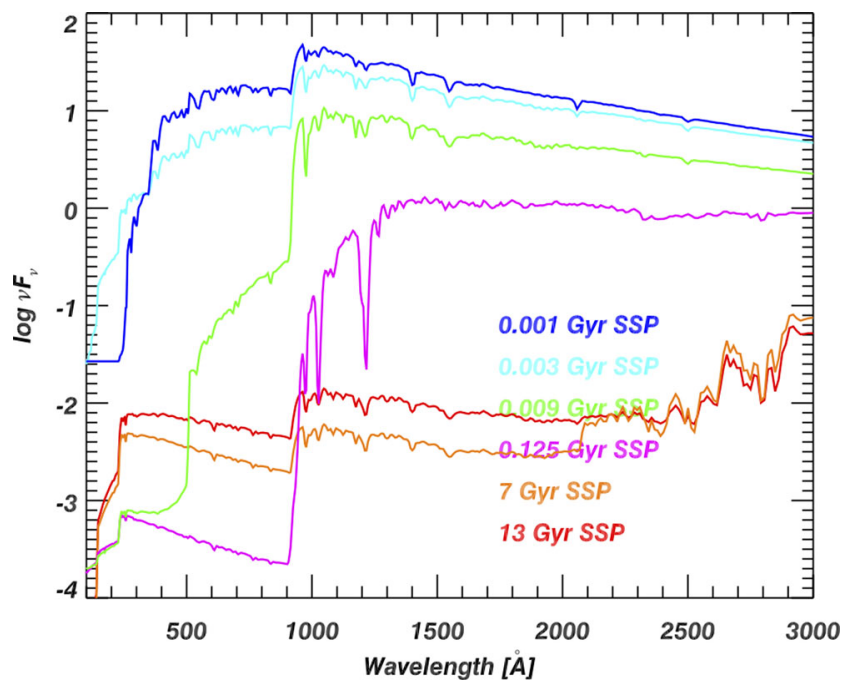

Figure 19. The incident spectra for SSP at $0.001,0.003,0.009,0.125,7$ and $13 \mathrm{Gyr}$ with solar metallicity, normalized to flux at $6000 \AA$. We can see that $\mathrm{OB}$ stars dominate the ionizing spectrum in the beginning and wane after tens of Myr. After that the ionizing spectrum is dominated by the emission from evolved stellar populations and it hardens. vertical direction (e.g. Ferguson et al. 1996; Zurita et al. 2000, 2002; Rossa \& Dettmar 2003a,b). In star-forming galaxies, hot evolved stars cannot compete with $\mathrm{O}$ stars in total ionizing photons production. However, we see in two cases that hot evolved stars might be a major contributor of ionizing photons. (1) In low-surface brightness regions that are located far away from $\mathrm{H}_{\text {II }}$ regions. The density of ionizing photons from the $\mathrm{H}$ II regions has dropped significantly so the hot evolved stars begin to dominate. One example is in regions at large vertical height (extra-planar gas at high $|z|$ ). Other low-surface brightness regions that show higher $[\mathrm{O} \mathrm{III}] / \mathrm{H} \beta$ than $\mathrm{H}$ II regions probably belong to this category as well. (2) In galaxies where OB stars have died: post-starburst galaxies and quiescent/passive galaxies. Quiescent galaxies are known to show LI(N)ER emissions (Sarzi et al. 2006; Yan et al. 2006; Stasińska et al. 2008; Kehrig et al. 2012; Singh et al. 2013; Belfiore et al. 2015, 2016a,b; Gomes et al. 2016). We predict DIG to be prevalent in post-starburst galaxies, and they will show LI(N)ER/AGN-like emission.

\section{IMPACT OF DIG ON METALLICITY MEASUREMENT}

There are several methods to measure gas-phase metallicity in starforming galaxies using strong lines in the optical, such as $\mathrm{N} 2=$ $[\mathrm{N} I I] / H \alpha, R_{23}=\left(\left[\mathrm{O}_{\mathrm{II}}\right] \lambda 3727+[\mathrm{O}\right.$ III $] \lambda 4959+[\mathrm{O}$ III] $\lambda 5007) / \mathrm{H} \beta$, $\mathrm{O} 3 \mathrm{~N} 2=(([\mathrm{O} \mathrm{III}] / \mathrm{H} \beta) /([\mathrm{N} I \mathrm{II}] / \mathrm{H} \alpha))$ (e.g. Alloin et al. 1979; Zaritsky, 

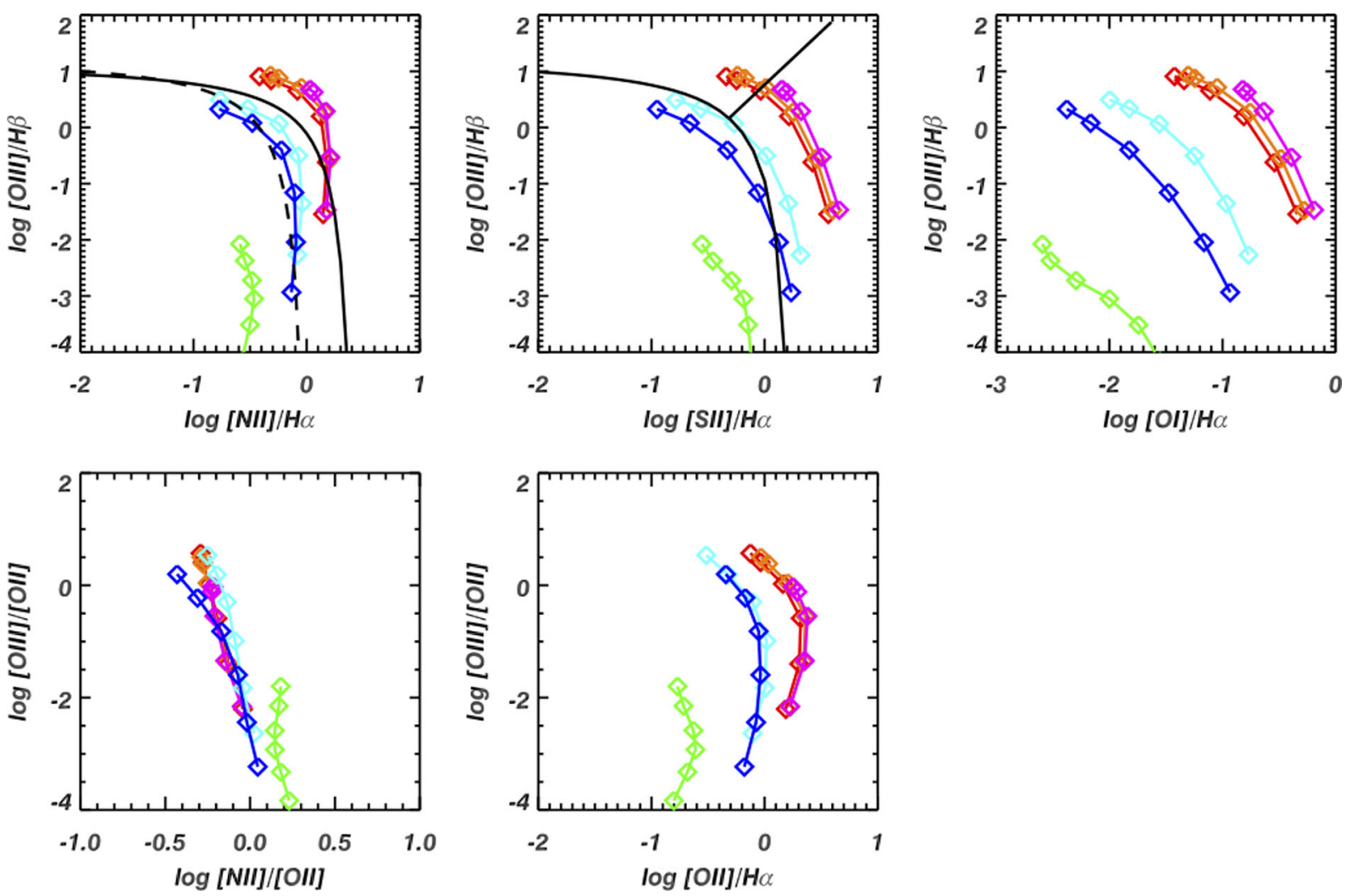

Figure 20. BPT diagrams, $\log \left[\mathrm{O}_{\mathrm{III}}\right] / \mathrm{H} \beta$ versus $\log \left[\mathrm{O}_{\mathrm{I}}\right] / \mathrm{H} \alpha, \log \left[\mathrm{O}_{\mathrm{III}}\right] /\left[\mathrm{O}\right.$ II] versus $\log \left[\mathrm{N}_{\mathrm{II}}\right] /\left[\mathrm{O}\right.$ II], and $\log \left[\mathrm{O}_{\mathrm{III}}\right] /\left[\mathrm{O}_{\mathrm{II}}\right]$ versus $\log \left[\mathrm{O}_{\mathrm{II}}\right] / \mathrm{H} \alpha$, for $\operatorname{different}$ incident spectra of SSPs at different ages. The colour scheme is the same as Fig. 19. The dots with the same colour have different ionization parameters from $\log U=-4.5$ to -2 with an interval of $0.5 \mathrm{dex}$. At Age $<$ tens of Myr, the ionizing spectrum softens as $\mathrm{OB}$ stars evolve and die. $\left[\mathrm{S}_{\text {II }}\right] / \mathrm{H} \alpha\left[\mathrm{N}_{\text {II }}\right] / \mathrm{H} \alpha\left[\mathrm{O}_{\text {II }}\right] / \mathrm{H} \alpha$ and $[\mathrm{O}$ III $] / \mathrm{H} \beta$, all decrease. After the $\mathrm{OB}$ stars die, the ionizing spectrum is dominated by hot evolved stars, and the spectrum hardens. We see increases of $\left[\mathrm{S}_{\mathrm{II}}\right] / \mathrm{H} \alpha\left[\mathrm{N}_{\mathrm{II}}\right] / \mathrm{H} \alpha\left[\mathrm{O}_{\mathrm{II}}\right] / \mathrm{H} \alpha$ and $[\mathrm{O} \mathrm{I}] / \mathrm{H} \alpha$. This figure shows how the ionized gas moves on the diagnostic diagrams as the stellar population ages.

Kennicutt \& Huchra 1994; Pilyugin 2001; Denicoló et al. 2002; Pettini \& Pagel 2004; Pilyugin \& Thuan 2005) and N2O2 = ([N $\left.{ }_{\text {II }}\right] /\left[\mathrm{O}_{\mathrm{II}}\right]$ ) (Dopita et al. 2000; Kewley et al. 2001), N2S2H $\alpha=$

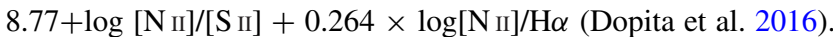
If the line ratios of DIG are different from these in $\mathrm{H}$ II regions, the metallicity measurement will inevitably be biased because these metallicity indicators are a combination of several line ratios. The bias depends on the fraction of emission contributed by DIG, which increases towards low-surface brightness regions.

\section{$5.1 \mathrm{~N} 2=\left[\mathrm{N}_{\text {III }}\right] / \mathrm{H} \alpha$}

If we use N2 to derive the metallicity, the enhancement seen in DIG means that the metallicity will be overestimated in those spaxels with a high DIG fraction. This is especially important for observation of galaxies at high redshift when only the emission lines near $\mathrm{H} \alpha$ are available. We see a typical $[\mathrm{N}$ II $] / \mathrm{H} \alpha$ enhancement of 0.2 dex. We use the equation in Pettini \& Pagel (2004) to convert $[\mathrm{N} \mathrm{II}] / \mathrm{H} \alpha$ to metallicity: $12+\log (\mathrm{O} / \mathrm{H})=9.37+2.03 \times N 2+1.26 \times N 2^{2}+0.32 \times N 2^{3}$, $\mathrm{N} 2=\log \left(\left[\mathrm{N}_{\mathrm{II}}\right] / \mathrm{H} \alpha\right)$. Assuming that an $\mathrm{H}_{\text {II }}$ region has $\mathrm{N} 2=-0.5$, $Z(\mathrm{~N} 2)=8.63$ in a local galaxy, we obtain $Z(\mathrm{~N} 2)=8.865$ for DIG with the same metallicity because $\mathrm{N} 2=-0.3$. The typical metallicity gradient for a local star-forming galaxy is about $-0.1 \mathrm{dex} R_{\mathrm{e}}{ }^{-1}$ (Sánchez et al. 2014; Ho et al. 2015), meaning that metallicity drops by $\sim 0.2$ from the centre to $2 R_{\mathrm{e}}$. The bias due to DIG would flatten the metallicity gradient from -0.1 dex $R_{\mathrm{e}}^{-1}$ to 0 if DIG totally dominates at $2 R_{\mathrm{e}}$. If we assume that a metal-poor $\mathrm{H}$ II region has
$\mathrm{N} 2=-1.5, Z(\mathrm{~N} 2)=8.08$ in a high-redshift galaxy, DIG with the same metallicity has $\mathrm{N} 2=-1.3$ and we would obtain $Z(\mathrm{~N} 2)=$ 8.157. The metallicity gradient will be flattened as well. N2 is usually used for measuring metallicity and metallicity gradients at high redshift (e.g. Wuyts et al. 2016). Our result indicates that the presence of DIG potentially flattens N2-derived metallicity gradients at high redshift.

\section{$5.2 \mathrm{~N} 2 \mathrm{O} 2=[\mathrm{N}$ II $] /[\mathrm{O} \mathrm{II}]$}

$\mathrm{N} 2 \mathrm{O} 2$ is a good metallicity indicator because it is not sensitive to ionization parameter or ionizing spectrum hardness but to metallicity (Dopita et al. 2000, 2013; Kewley \& Dopita 2002). The caveat of $\mathrm{N} 2 \mathrm{O} 2$ is that it relies on the N/O. Nitrogen and $\alpha$ elements have different enrichment time-scales, hence their ratio depends on the star formation history and several other parameters (e.g. Vincenzo et al. 2016) as well as mixing issues (e.g. Belfiore et al. 2015), especially for low-mass galaxies and galaxy outskirts, where there are prominent variations of $\mathrm{N} / \mathrm{O}$ versus $\mathrm{O} / \mathrm{H}$ relative to metal-rich systems and central regions. Moreover, these diagnostics are sensitive to metallicity only at $12+\log (\mathrm{O} / \mathrm{H})>8.3$, i.e. where $\mathrm{N} / \mathrm{O}$ is a strong function of metallicity, but they become essentially insensitive to metallicity at $12+\log (\mathrm{O} / \mathrm{H})<8.3$, i.e. in the regime where $\mathrm{N} / \mathrm{O}$ is nearly constant. We plot metallicities derived using $\mathrm{N} 2 \mathrm{O} 2=[\mathrm{N} I] /\left[\mathrm{O}_{\mathrm{II}}\right]$ as a function of effective radius and colour-code the dots with $\mathrm{H} \alpha$ surface brightness in Fig. 21. N2O2 is converted to metallicity using $\log (\mathrm{O} / \mathrm{H})+12=$ $\log \left[1.54020+1.26602 \times R+0.167977 \times R^{2}\right]+8.93$, 

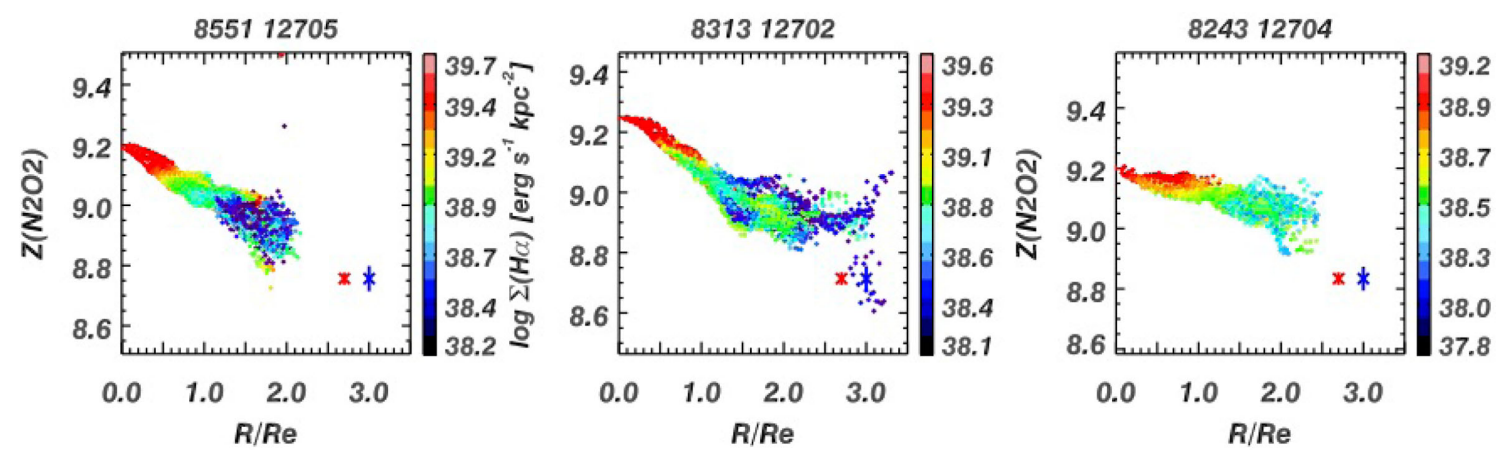

Figure 21. Metallicity derived using $\mathrm{N} 2 \mathrm{O} 2$ as a function of radius. $Z(\mathrm{~N} 2 \mathrm{O} 2)$ drops with radius due to a metallicity gradient. We see that the derived metallicity does not depend on the $\Sigma_{\mathrm{H} \alpha}$ at a fixed radius, and the dispersion at a fixed radius is similar to the measuring error. Red and blue bars are errors at log $\Sigma_{\mathrm{H} \alpha}=39$ and $38.5 \mathrm{erg} \mathrm{s}^{-1} \mathrm{kpc}^{-2}$, respectively. The length of the error bars is $2 \sigma$. We show in Section 5.7 that the impact of DIG is prevalent in all star-forming galaxies in our sample, not confined to the three galaxies we show.

$R=\log [\mathrm{N}$ II]/[O II] (Dopita et al. 2013). At fixed radius, $Z(\mathrm{~N} 2 \mathrm{O} 2)$ in the low-surface brightness bin is similar to or a little bit higher than that in the high-surface brightness bin. As stated in Section 3.2, $[\mathrm{N}$ II]/[O II] only depends on the N/O abundance ratio and temperature (Dopita et al. 2000, 2013). It is not subject to the ionization parameter and ionizing spectrum shape variation. This makes it an excellent metallicity indicator. The weak dependence of $[\mathrm{N}$ II $] /\left[\mathrm{O}_{\mathrm{II}}\right]$ on $\mathrm{H} \alpha$ surface brightness at fixed radius (Fig. 21) demonstrates that it is a good metallicity indicator even in the presence of DIG. However, we note that $\mathrm{N} 2 \mathrm{O} 2$ is subject to the variation of N/O ratio (PérezMontero \& Contini 2009; Pérez-Montero et al. 2013, 2016), temperature variation and uncertainty of extinction correction. $Z(\mathrm{~N} 2 \mathrm{O} 2)$ could be enhanced or suppressed for DIG in some regions, maybe due to $\mathrm{N} / \mathrm{O}$ variation and temperature variation. The measurement of extinction involves an accurate measurement of $\mathrm{H} \beta$, which is not an easy task since the stellar continuum shows deep $\mathrm{H} \beta$ absorption. This is even harder when the emission line is weak and $\mathrm{S} / \mathrm{N}$ is low, such as in the outskirts of galaxies. Besides, $\mathrm{H} \alpha$ and $\mathrm{H} \beta$ arise from the fully ionized region, while $[\mathrm{N} \mathrm{II}]$ and $[\mathrm{O}$ II] are from the partially ionized region. The extinction derived using $\mathrm{H} \alpha$ and $\mathrm{H} \beta$ may not necessarily be the same as the extinction experienced by the partially ionized region. From Fig. 6, we do not see any signs of deviation of $\mathrm{H} \beta / \mathrm{H} \alpha$ for different $\mathrm{H} \alpha$ surface brightness spaxels, so the result that DIG have similar $\mathrm{N} 2 \mathrm{O} 2$ as $\mathrm{H}$ II region is robust. We see in Fig. 6 that $\mathrm{H} \beta / \mathrm{H} \alpha$ shows a gradient with positive slope even though it does not depend on $\Sigma_{\mathrm{H} \alpha}$. The gradient in $\mathrm{H} \beta / \mathrm{H} \alpha$ will change the $\mathrm{Z}(\mathrm{N} 2 \mathrm{O} 2)$ gradient slope. To correct this, we suggest getting an $\mathrm{H} \beta / \mathrm{H} \alpha$ gradient by fitting the $\mathrm{H} \beta / \mathrm{H} \alpha$ of individual spaxels and apply the overall extinction gradient to $\left[\mathrm{N}_{\text {II }}\right] /\left[\mathrm{O}_{\mathrm{II}}\right]$ to get the correct metallicity gradient. In this way, we avoid the very noisy $\mathrm{H} \beta / \mathrm{H} \alpha$ when $\Sigma_{\mathrm{H} \alpha}$ is low when correcting extinction.

A key for probing abundance is to use lines from different elements with the same ionization potential. The ionization potential for $H^{0}, O^{0}, O^{+}, O^{++}, S^{+}$and $N^{+}$are $13.598,13.618,35.121,54.936$, 23.337 and $29.601 \mathrm{eV}$ respectively. Ionization potential of [O II] and [N ${ }_{\text {II] }}$ are similar: 35.121 and $29.601 \mathrm{eV}$. This makes $[\mathrm{N}$ II] $/[\mathrm{O}$ II] insensitive to ionization parameter, thus a good metallicity indicator. The complexity in using $[\mathrm{N}$ II]/[O $\mathrm{II}]$ is the extinction correction and the $\mathrm{N} / \mathrm{O}$ ratio variation at fixed $\mathrm{O} / \mathrm{H}$.

To quantify the change of the metallicity measurements as a function of $\mathrm{H} \alpha$ surface brightness, we do a linear regression fit to the derived metallicities versus $\mathrm{H} \alpha$ surface brightness relation in a narrow annulus. The distribution of slopes for different metallicity indicators at three radii are shown in black, blue and red in Fig. 29. The metallicity is independent of surface brightness. The reason we are seeing a dependence of metallicity on $\mathrm{H} \alpha$ surface brightness is due to contamination by DIG. For $Z(\mathrm{~N} 2 \mathrm{O} 2)$, the slope distribution is narrow and peaks at $\sim 0.05$. The bias in metallicity using $\mathrm{N} 2 \mathrm{O} 2$ is small and the error is small because $\mathrm{N} 2 \mathrm{O} 2$ does not vary much among DIG. DIG has marginally lower $Z(\mathrm{~N} 2 \mathrm{O} 2)$, suggesting marginally higher temperature.

\section{$5.3 R_{23}$}

$R_{23}=([\mathrm{O}$ II $] \lambda 3727+[\mathrm{O}$ III $] \lambda 4959+[\mathrm{O}$ III $] \lambda 5007) / \mathrm{H} \beta$ is a commonly used metallicity indicator (McGaugh 1991; Zaritsky et al. 1994; Pilyugin 2001; Kewley \& Dopita 2002; Kobulnicky \& Kewley 2004). The advantage is it does not depend on the N/O ratio. The disadvantage is that the metallicity is double-valued at a given $R_{23}$. In order to use $R_{23}$ to measure $Z$, one has to first determine the ionization parameter and which branch it is on. Therefore, it always needs to be used in conjunction with $\left[\mathrm{O}_{\text {III }}\right] /[\mathrm{O}$ II] and N2O2 (or similar). We use the method of Kewley \& Ellison (2008) to determine which branch a spaxel is on and derive the metallicity. Fig. 22 shows how the $Z$ derived from $R_{23}$ changes with radius and $\mathrm{H} \alpha$ surface brightness. One can see that low-SB regions have lower measured $Z\left(R_{23}\right)$ values. We have shown in Section 3.2 that DIG has higher $[\mathrm{O} I] / \mathrm{H} \beta$, lower $\left[\mathrm{O}_{\mathrm{III}}\right] /[\mathrm{O} \mathrm{II}]$ and, on average, similar $\left[\mathrm{O}_{\mathrm{III}}\right] / \mathrm{H} \beta$ as $\mathrm{H}$ II regions. The combination of the three line ratios leads to the result that $Z\left(R_{23}\right)$ is biased to be lower in DIG.

For the whole sample, the $Z\left(R_{23}\right)$ versus $\Sigma_{\mathrm{H} \alpha}$ slope distribution peaks at $\sim 0.2$ and the dispersion is large. The contribution of DIG would not only bias the metallicity measurement systematically, but also introduce large metallicity measurement uncertainties, as the scatter in the metallicity versus radius relation is large. We note that the gas-phase metallicity of star-forming galaxies shows a universal gradient of -0.1 dex $R_{\mathrm{e}}{ }^{-1}$. Meanwhile, the slope of $Z\left(R_{23}\right)$ versus $\Sigma_{\mathrm{H} \alpha}$ is $\sim 0.2$. According to Fig. $22, \Sigma_{\mathrm{H} \alpha}$ of DIG at $2 R_{\mathrm{e}}$ is about $38 \mathrm{erg} \mathrm{s}^{-1} \mathrm{kpc}^{-2}$. Compared to a $\Sigma_{\mathrm{H} \alpha}=39 \mathrm{erg} \mathrm{s}^{-1} \mathrm{kpc}^{-2} \mathrm{H}_{\text {II }}$ region, the $Z\left(R_{23}\right)$ bias for DIG would be 0.2. Note the drop of metallicity from the centre to $2 R_{\mathrm{e}}$ is only $0.2 \mathrm{dex}$. The metallicity gradient would be -0.2 dex $R_{\mathrm{e}}{ }^{-1}$ if the outskirt of the galaxy is pure DIG. In other words, the metallicity measured using $Z\left(R_{23}\right)$ is biased systematically by $\sim-0.1 \operatorname{dex} R_{\mathrm{e}}^{-1}$. 

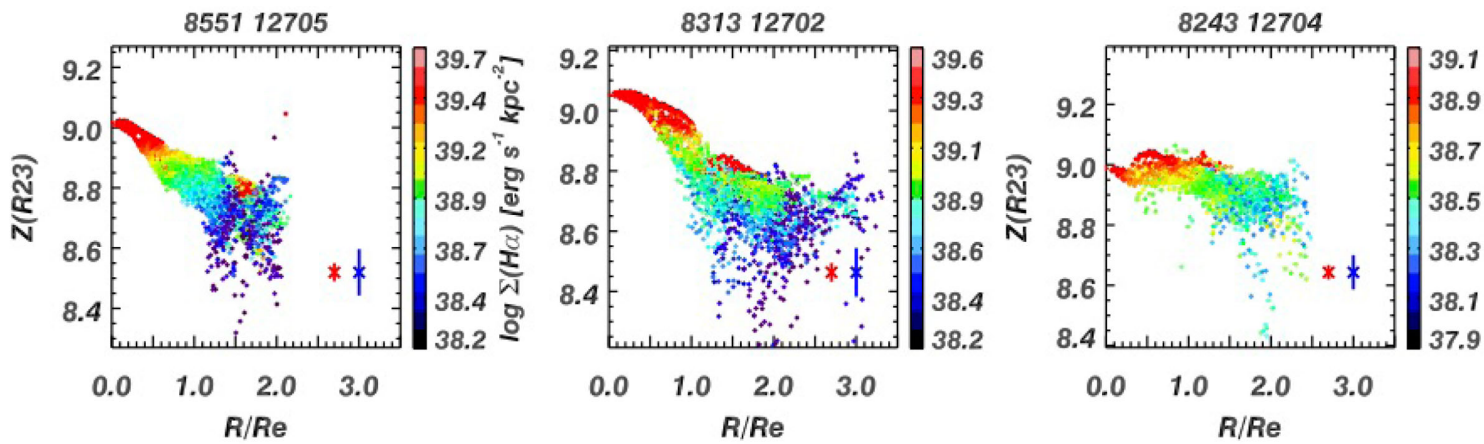

Figure 22. Metallicity derived using $R_{23}$ as a function of radius. $Z\left(R_{23}\right)$ drops with radius due to metallicity gradient. At fixed radius, $Z\left(R_{23}\right)$ decreases with $\Sigma_{\mathrm{H} \alpha}$, indicating $Z\left(R_{23}\right)$ is systematically biased low for DIG. The measuring errors at log $\Sigma_{\mathrm{H} \alpha}=39$ and $38.5 \mathrm{erg} \mathrm{s}^{-1} \mathrm{kpc}^{-2}$ are indicated using red and blue bars. The dispersion of $Z\left(R_{23}\right)$ is about twice that of $Z(\mathrm{~N} 2 \mathrm{O} 2)$. The metallicity gradient would be systematically biased by -0.1 dex $R_{\mathrm{e}}{ }^{-1}$ if the bias in DIG is not accounted for.
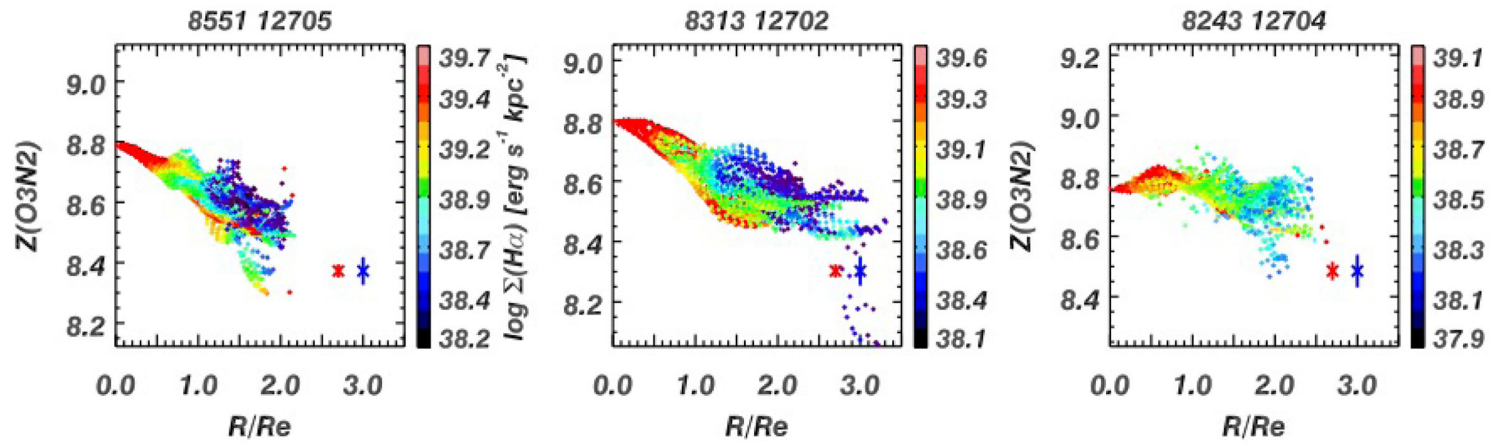

Figure 23. Metallicity derived using $\mathrm{O} 3 \mathrm{~N} 2$ as a function of radius. $Z(\mathrm{O} 3 \mathrm{~N} 2)$ drops with radius due to a metallicity gradient. At fixed radius, $Z(\mathrm{O} 3 \mathrm{~N} 2)$ could either decrease or increase with $\Sigma_{\mathrm{H} \alpha}$. The measuring errors at $\log \Sigma_{\mathrm{H} \alpha}=39$ and $38.5 \mathrm{erg} \mathrm{s}^{-1} \mathrm{kpc}^{-2}$ are indicated using red and blue bars. The dispersion of $Z(\mathrm{O} 3 \mathrm{~N} 2)$ is similar to that of $Z(\mathrm{~N} 2 \mathrm{O} 2)$. The metallicity gradient would be biased by $\pm 0.05 R_{\mathrm{e}}{ }^{-1}$ if the bias in DIG is not considered.

\section{$5.403 N 2$}

O3N2 is sensitive to oxygen abundance and it is not impacted by extinction. The disadvantage of $\mathrm{O} 3 \mathrm{~N} 2$ is that it depends on $\mathrm{N} / \mathrm{O}$ (Pérez-Montero \& Contini 2009; Pérez-Montero et al. 2013, 2016) and the ionization parameter. In Fig. 23, we show how DIG would impact metallicity measurements made from $\mathrm{O} 3 \mathrm{~N} 2=$ $([\mathrm{O} I I] / H \beta) /\left(\left[\mathrm{N}_{\mathrm{II}}\right] / \mathrm{H} \alpha\right)$. We see in Fig. 23 that $\mathrm{O} 3 \mathrm{~N} 2$ is higher in DIG for some galaxies and lower or similar in other galaxies. The contamination by DIG could be responsible for a substantial portion of the scatter in metallicity measurements. When confined only to the high-surface brightness regions, the metallicity gradient derived using O3N2 is similar to the ones using $R_{23}$ or N2O2. To make robust metallicity gradient measurements, one has to properly isolate $\mathrm{H}$ II regions and correct for DIG contamination.

Because the surface brightness generally decreases towards large radii, the metallicity gradient derived using $\mathrm{O} 3 \mathrm{~N} 2$ at large radii might be flatter or steeper than that derived using $\mathrm{N} 2 \mathrm{O} 2$ if we include all the spaxels. For example, in MaNGA galaxy 8313-12702, we get a metallicity of $-0.15 \operatorname{dex} R_{\mathrm{e}}^{-1}$ using Z(N2O2). Using only the highsurface brightness regions (red dots) and $Z(\mathrm{O} 3 \mathrm{~N} 2)$ gives the same result. However, if we include everything, the metallicity gradient derived using $\mathrm{O} 3 \mathrm{~N} 2$ will be $-0.1 \operatorname{dex} R_{\mathrm{e}}^{-1}$. The bias is significant at least for this galaxy. $Z(\mathrm{O} 3 \mathrm{~N} 2)$ is biased in the other direction for MaNGA galaxy 8603-12704. Similarly, Mast et al. (2014) found that the presence of DIG would bias the abundance gradient when using $\mathrm{O} 3 \mathrm{~N} 2$ to derive metallicity. The strength of the bias depends a lot on the assumed calibrator. We note the degree of bias is not the same for all galaxies and we definitely can give a robust metallicity gradient measurement using high-surface brightness regions. The $\sigma$ of the distribution of $Z(\mathrm{O} 3 \mathrm{~N} 2)$ versus $\Sigma_{\mathrm{H} \alpha}$ slope at fixed radius is $\sim 0.1$. This translates to a metallicity gradient dispersion of \pm 0.05 dex $R_{\mathrm{e}}{ }^{-1}$ through a similar analysis as in Section 5.3, consistent with the different biases we see in different galaxies. For a large sample of galaxies (Sánchez et al. 2014; Ho et al. 2015), the bias for different galaxies may cancel out when deriving average metallicity gradients.

\section{$5.5 \mathrm{~N} 2 \mathrm{~S} 2 \mathrm{H} \alpha$}

Dopita et al. (2016) proposed a new metallicity proxy using only [N $\mathrm{NI}] \lambda 6548$, [S II] $\lambda \lambda 6717,6731$, and $\mathrm{H} \alpha: 12+\log (\mathrm{O} / \mathrm{H})=$ $\mathrm{Z}(\mathrm{N} 2 \mathrm{~S} 2 \mathrm{H} \alpha)=8.77+\log [\mathrm{N}$ II $] /[\mathrm{S}$ II $]+0.264 \times \log [\mathrm{N}$ II $] / \mathrm{H} \alpha$. It is especially suitable for metallicity measurements of high-redshift galaxies whose spectral wavelength coverage is limited because the four lines used are close to each other. This estimator is almost linear up to an abundance of $12+\log (\mathrm{O} / \mathrm{H})=9.05$. The caveat is the calibration of this metallicity proxy is based on a well-defined $\mathrm{N} / \mathrm{O}$ and $\mathrm{O} / \mathrm{H}$ relation. This calibration fails for any systems deviating from the assumed N/O-O/H relation. We explore how DIG biases the metallicities derived using $\mathrm{N} 2 \mathrm{~S} 2 \mathrm{H} \alpha$ in Fig. 24. At fixed radius, we do not see a significant offset in $Z(\mathrm{~N} 2 \mathrm{~S} 2 \mathrm{H} \alpha)$ between DIG and $\mathrm{H}$ II regions. This is because $Z(\mathrm{~N} 2 \mathrm{~S} 2 \mathrm{H} \alpha)$ is insensitive to variation of ionization parameter and spectral hardness (Dopita et al. 2016), very similar to $\mathrm{N} 2 \mathrm{O} 2$. Compared with $Z(\mathrm{~N} 2 \mathrm{O} 2)$, the 

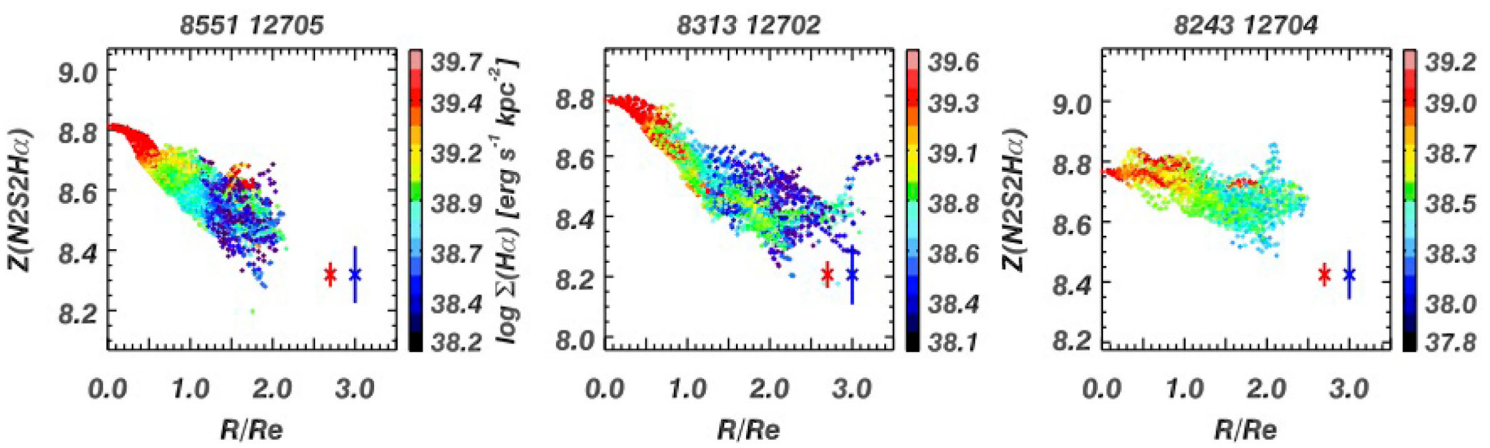

Figure 24. Metallicity derived using [N $\mathrm{II}] /\left[\mathrm{S}_{\mathrm{II}}\right]$ and $\left[\mathrm{O}_{\mathrm{II}}\right] / \mathrm{H} \alpha$ proposed by Dopita et al. (2016) as a function of radius. We see that the derived metallicity does not depend on the $\Sigma_{\mathrm{H} \alpha}$ at a fixed radius and the dispersion at a fixed radius is similar to the measuring error (red and blue bars are errors at log $\Sigma_{\mathrm{H} \alpha}=39$ and $38.5 \mathrm{erg} \mathrm{s}^{-1} \mathrm{kpc}^{-2}$, respectively). $Z(\mathrm{~N} 2 \mathrm{~S} 2 \mathrm{H} \alpha)$ performs very similar to $Z(\mathrm{O} 3 \mathrm{~N} 2)$.
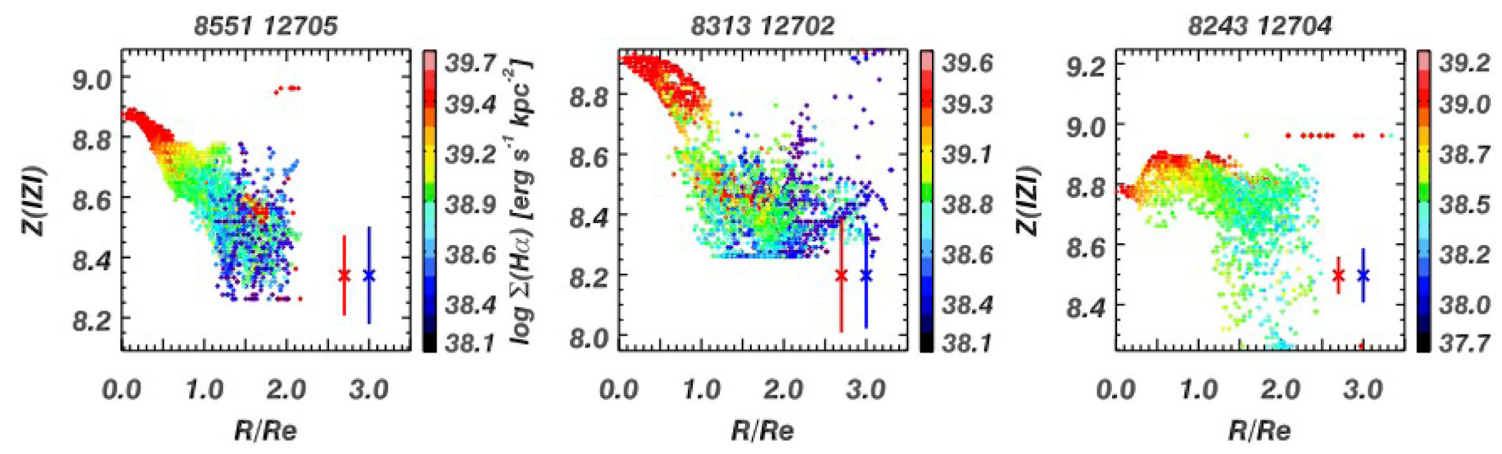

Figure 25. Metallicity derived using IZI by Blanc et al. (2015) as a function of radius. The dots are colour-coded by $\Sigma_{\mathrm{H} \alpha}$. For high $\Sigma_{\mathrm{H} \alpha}$ regions, the metallicity gradients are similar to those derived using other metallicity indicators. However, at fixed radius, different surface brightness regions have large metallicity discrepancies. For low $\Sigma_{\mathrm{H} \alpha}$ regions, the metallicities derived from IZI can vary by 0.2 dex themselves. This demonstrates that IZI is not robust to derive the metallicity of DIG. Note that the metallicity lower limit is set to 8 , so the cut-off at $Z \sim 8.3$ is not due to a high-metallicity lower limit.

dispersion of $Z(\mathrm{~N} 2 \mathrm{~S} 2 \mathrm{H} \alpha)$ is larger. This is understandable because of the following.

(i) Four lines are used in $Z(\mathrm{~N} 2 \mathrm{~S} 2 \mathrm{H} \alpha)$ while only two lines are used to derive $Z(\mathrm{~N} 2 \mathrm{O} 2)$. The measurement errors enter into the derivation of metallicities.

(ii) The relative abundances of $\mathrm{N}, \mathrm{S}$ and $\mathrm{O}$ are involved in $Z(\mathrm{~N} 2 \mathrm{~S} 2 \mathrm{H} \alpha)$ while $Z(\mathrm{~N} 2 \mathrm{O} 2)$ only include N/O.

According to the upper right panel of Fig. 29, The distribution of $Z(\mathrm{~N} 2 \mathrm{~S} 2 \mathrm{H} \alpha)$ versus $\Sigma_{\mathrm{H} \alpha}$ slope is very similar to that of $Z(\mathrm{O} 3 \mathrm{~N} 2)$ in its centroid and dispersion. The slope distribution for $Z(\mathrm{~N} 2 \mathrm{~S} 2 \mathrm{H} \alpha)$ has a wider wing, meaning that there are some sources that show significant biases in $Z(\mathrm{~N} 2 \mathrm{~S} 2 \mathrm{H} \alpha)$ for DIG and $\mathrm{H}$ II regions. This is similar to the scatter introduced by DIG on O3N2.

\subsection{IZI}

IZI (Blanc et al. 2015) uses strong nebular emission lines to derive the Bayesian posterior probability density function for metallicity and ionization parameter based on a series of $\mathrm{H}$ II region models. In Fig. 25, we plot the metallicities derived using IZI as a function of radius. We input [O II] $\lambda 3727,[\mathrm{O}$ III] $\lambda 4959,[\mathrm{O}$ III] $\lambda 5007, \mathrm{H} \alpha,[\mathrm{N} \mathrm{II}]$ $\lambda 6583$ and $\left[\mathrm{S}_{\mathrm{II}}\right] \lambda \lambda 6717,6731$ for the calculation. All the emissionline fluxes are corrected for reddening using the Balmer decrement $\mathrm{H} \alpha / \mathrm{H} \beta$ assuming an MW extinction curve (Fitzpatrick 1999). We use the values derived with the 'output joint mode' in IZI. The dots are colour-coded by $\Sigma_{\mathrm{H} \alpha}$. For high $\Sigma_{\mathrm{H} \alpha}$ regions, IZI gives similar metallicity gradients as the other metallicity indicators. However, at a fixed radius, different surface brightness regions (different colours in the plots) have large metallicity discrepancies. For low $\Sigma_{\mathrm{H} \alpha}$ regions, the metallicities derived from IZI can vary by $0.2 \mathrm{dex}$ among themselves. This is at least partly because IZI currently only adopts $\mathrm{H}_{\text {II }}$ region grids. These models make an inherent assumption that the ionizing spectrum shape is fixed by the temperature of the OB stars, which is determined by the metallicity. However, for DIG, this assumption does not hold. We have shown that metallicity+low $q$ for an $\mathrm{H}$ II region model cannot produce the line ratios we see in DIG. Thus, IZI is very vulnerable to contamination by DIG because currently it only contains $\mathrm{H}$ II region models. However, if one were to include a DIG model in IZI and other Bayesian codes to derive metallicities (e.g.HII-CHI-mistry: Pérez-Montero 2014; BOND: Vale Asari et al. 2016), then they may be able to give accurate estimates of metallicity and ionization parameter even with the contamination of DIG.

Furthermore, we input only extinction-corrected [N $\mathrm{II}]$ and [O II] into IZI to see if IZI could give a less biased metallicity in DIG. [N $\mathrm{II}]$ and [O II] can only be combined to $\mathrm{N} 2 \mathrm{O} 2$ to estimate metallicity. Figs 26 and 27 show that the metallicities derived through IZI using [N $\mathrm{NI}]$ and [O $\mathrm{OII}_{\mathrm{II}}$ only and [N $\left.\mathrm{NI}\right],\left[\mathrm{O}_{\mathrm{II}}\right]$ and [O $\left.\mathrm{OII}\right]$ are better than metallicity derived using all strong emission lines available. The dispersion at a fixed radius is smaller and the dependence on $\Sigma_{\mathrm{H} \alpha}$ at a fixed radius is weaker. However, $Z(\mathrm{~N} 2 \mathrm{O} 2)$ in Fig. 21 still shows a 

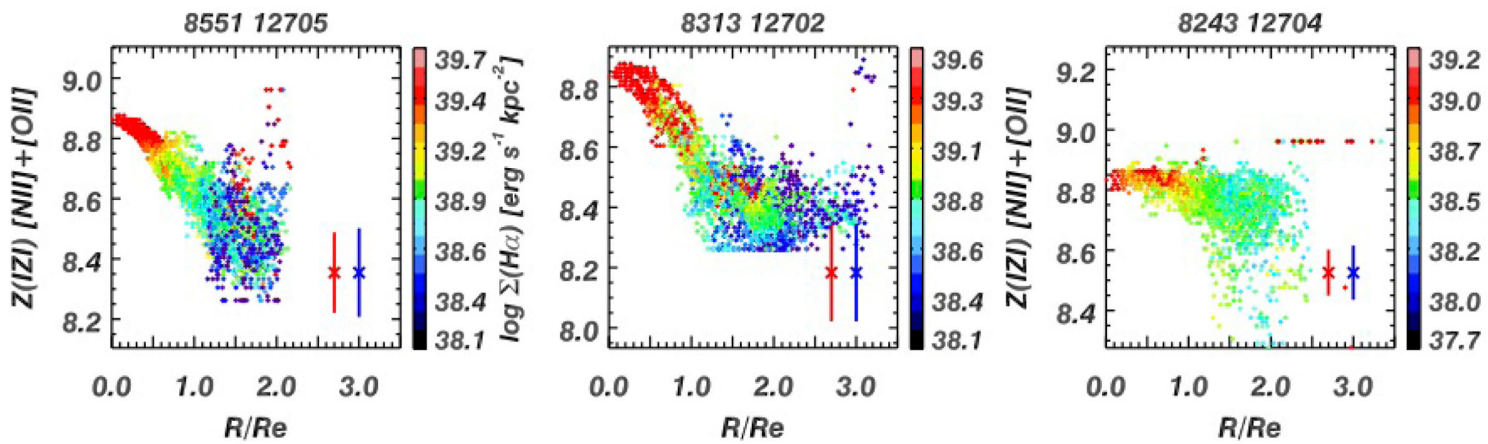

Figure 26. Metallicity derived using IZI by Blanc et al. (2015) as a function of radius using only extinction-corrected [ $\mathrm{N}_{\text {II] }}$ and [O II]. The impact of DIG is much less than when using all strong emission lines available. The red and blue bars show the typical line ratio errors at $\log \Sigma_{\mathrm{H} \alpha}=39$ and $38.5 \mathrm{erg} \mathrm{s}^{-1} \mathrm{kpc}^{-2}$ for individual spaxels. The length of the error bars is $2 \sigma$.
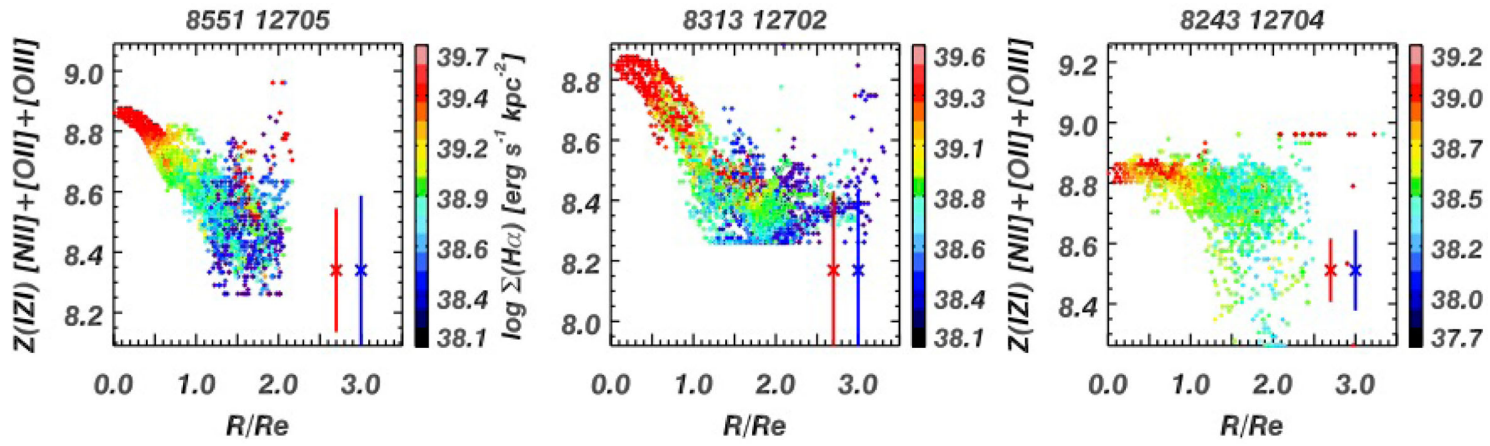

Figure 27. Metallicity derived using IZI by Blanc et al. (2015) as a function of radius using only extinction-corrected [N III], [O II] and [O III]. The result is indistinguishable from the result using only [ $\left.\mathrm{N}_{\mathrm{II}}\right]$ and $[\mathrm{O}$ II]. The impact of DIG is much less than when using all strong emission lines available. The red and blue bars show the typical line ratio error at $\log \Sigma_{\mathrm{H} \alpha}=39$ and $38.5 \mathrm{erg} \mathrm{s}^{-1} \mathrm{kpc}^{-2}$ for individual spaxels. The length of the error bars is $2 \sigma$.

tighter metallicity gradient than $Z(\mathrm{IZI})[\mathrm{N}$ II $]+\left[\mathrm{O}_{\mathrm{II}}\right]$. This is because we do not apply extinction corrections to individual spaxels when calculating $Z(\mathrm{~N} 2 \mathrm{O} 2)$. IZI metallicity with extinction-uncorrected $[\mathrm{N}$ II] and [O II] input alone gives very similar result as $Z(\mathrm{~N} 2 \mathrm{O} 2)$.

\subsection{Comparison of different metallicity estimators}

\subsubsection{Metallicities versus $\Sigma_{\mathrm{H} \alpha}$ relation: the whole sample}

To quantify the bias introduced by DIG on strong-line metallicity measurements, we examine the derived metallicities as a function of $\Sigma_{\mathrm{H} \alpha}$ for the whole sample. We select all spaxels in $\left[0.4 R_{\mathrm{e}}, 0.6 R_{\mathrm{e}}\right]$, subtract from all metallicity and $\log \Sigma_{\mathrm{H} \alpha}$ measurements their respective medians then plot all galaxies together in the same plot. The spaxels in each galaxy are weighted by $\frac{1}{N}$, where $N$ is the number of valid spaxels in this galaxy. Fig. 28 shows the weighted $\Delta Z$ versus $\Delta \log \Sigma_{\mathrm{H} \alpha}$ relation for the whole sample. A non-zero slope of the relation means that metallicities are biased by DIG contribution, and the dispersion of this relation in $y$-direction reflects how reliable a metallicity proxy is for an individual galaxy. The $\Delta Z(\mathrm{~N} 2 \mathrm{O} 2)$ versus $\Delta \Sigma_{\mathrm{H} \alpha}$ relation has a slope of $\sim 0.05$. The slope of $\Delta Z\left(R_{23}\right)$ versus $\Delta \log \Sigma_{\mathrm{H} \alpha}, \Delta Z(\mathrm{O} 3 \mathrm{~N} 2)$ versus $\Delta \log \Sigma_{\mathrm{H} \alpha}, \Delta Z(\mathrm{~N} 2 \mathrm{~S} 2 \mathrm{H} \alpha)$ versus $\Delta \log \Sigma_{\mathrm{H} \alpha}, \Delta Z(\mathrm{~N} 2)$ versus $\Delta \log \Sigma_{\mathrm{H} \alpha}$, relations are $\sim 0.2,-0.05$, $\sim 0$ and -0.2 . We define the dispersion as the interval between 90 per cent percentiles contours at $\Delta \log \Sigma_{\mathrm{H} \alpha}=0$. For $Z(\mathrm{~N} 2 \mathrm{O} 2)$, the dispersion is 0.2 , and for $Z\left(R_{23}\right), Z(\mathrm{O} 3 \mathrm{~N} 2), Z(\mathrm{~N} 2 \mathrm{~S} 2 \mathrm{H} \alpha)$ and $Z(\mathrm{~N} 2)$, the dispersions are $0.35,0.23,0.37$ and 0.25 . Based on slopes and dispersions, $Z(\mathrm{~N} 2 \mathrm{O} 2)$ is optimal because the slope is mild and the dispersion is smallest. $Z\left(R_{23}\right)$ has the most significant slope and large dispersion. $Z(\mathrm{O} 3 \mathrm{~N} 2)$ has the smallest slope and a dispersion only slightly larger than $Z(\mathrm{~N} 2 \mathrm{O} 2) . Z(\mathrm{~N} 2 \mathrm{~S} 2 \mathrm{H} \alpha)$ has a nearly 0 slope but the dispersion is the largest. $Z(\mathrm{~N} 2)$ is derived using $[\mathrm{N} I] / \mathrm{H} \alpha$, thus very vulnerable to DIG contamination.

We also do linear regression to the derived metallicities versus $\log \Sigma_{\mathrm{H} \alpha}$ relation in narrow radial annuli so the metallicity is essentially fixed. The distribution of slopes for different metallicity indicators at three radii are shown in black, blue and red in Fig. 29. The intrinsic metallicity is independent of surface brightness. The reason we are seeing a dependence of derived metallicities on $\mathrm{H} \alpha$ surface brightness is due to contamination by DIG. A slope of 0 means the DIG does not impact the metallicity measurements and a positive slope means DIG biases the metallicity measurement low. The centroid of the distribution reflects the systematic offset of that metallicity indicator for the whole sample, while the dispersion of the distribution reflects the metallicity error it brings for the individual galaxies. For $Z(\mathrm{~N} 2 \mathrm{O} 2)$, the slope distribution is narrow and peaks at $\sim 0.05 \mathrm{dex}^{-1}$. The bias in metallicity using $\mathrm{N} 2 \mathrm{O} 2$ is small and the error is small because $\mathrm{N} 2 \mathrm{O} 2$ does not vary much among DIG. DIG regions have marginally lower $Z(\mathrm{~N} 2 \mathrm{O} 2)$, suggesting slightly higher temperature. The slope distribution of $Z(\mathrm{~N} 2 \mathrm{~S} 2 \mathrm{H} \alpha)$ is very similar to that of $Z(\mathrm{~N} 2 \mathrm{O} 2)$, suggesting $Z(\mathrm{~N} 2 \mathrm{~S} 2 \mathrm{H} \alpha)$ to be a robust metallicity estimator even in the presence of DIG. For $Z\left(R_{23}\right)$, the slope distribution peaks at $0.2 \mathrm{dex}^{-1}$ and dispersion is large. The contribution of DIG would not only bias the metallicity measurements 

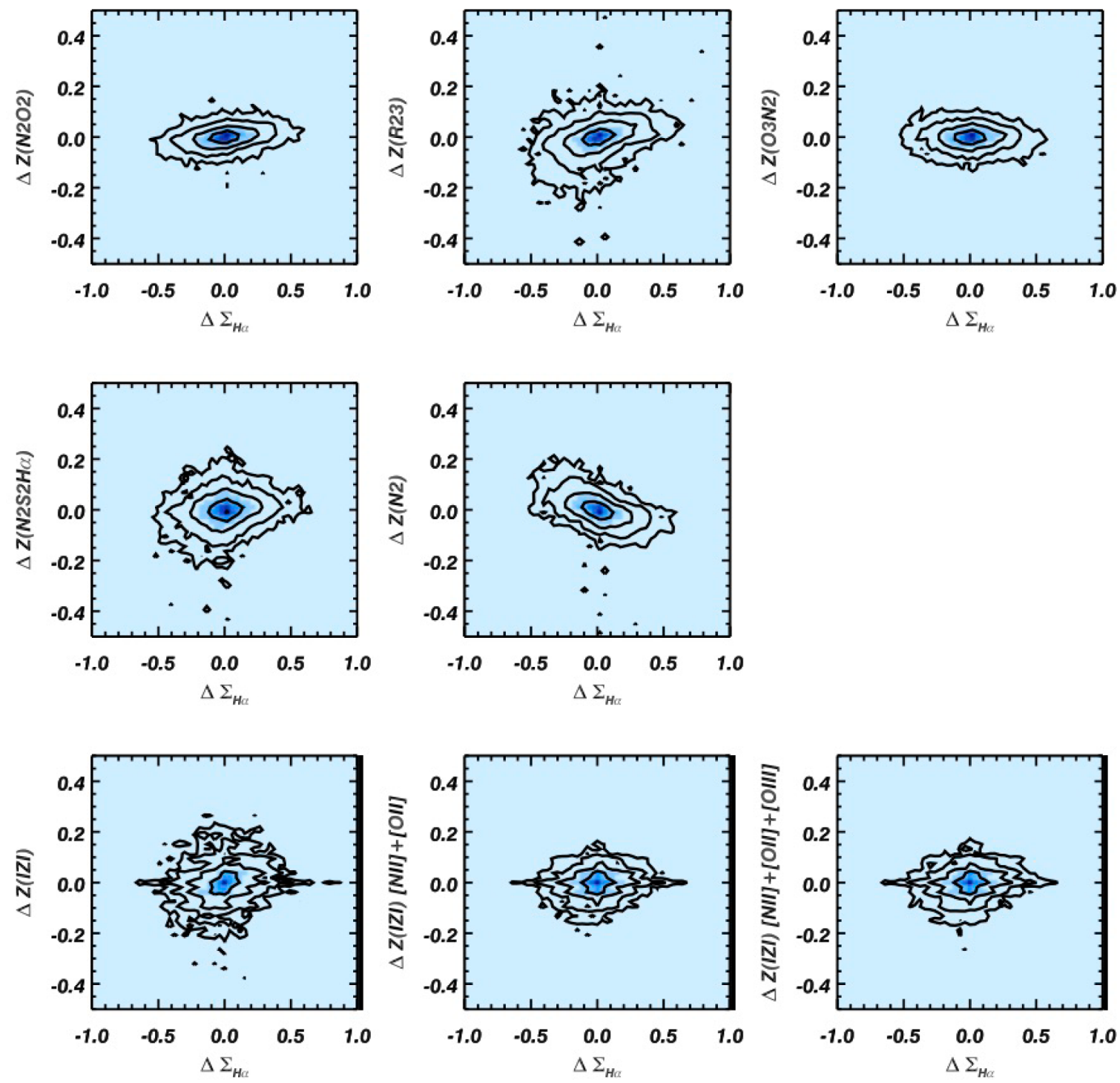

Figure 28. $\Delta Z$ versus $\Delta \log \Sigma_{\mathrm{H} \alpha}$ relation at $\left[0.4 R_{\mathrm{e}}, 0.6 R_{\mathrm{e}}\right]$ for all galaxies. We normalize the $\Delta Z$ versus $\Delta \log \Sigma_{\mathrm{H} \alpha}$ relation by subtracting the median $Z$ and median $\log \Sigma_{\mathrm{H} \alpha}$. All the spaxels in a galaxy are weighted by $\frac{1}{N}$, where $N$ is the number of valid spaxels in the galaxy. A non-zero slope of the relation means that metallicities are biased by DIG contribution, and the dispersion of this relation in $y$-direction reflects how reliable a metallicity proxy is for an individual galaxy.

systematically, but also introduce a large metallicity measurement error. This can be seen in the tightness of metallicity versus radius relation. For $Z(\mathrm{O} 3 \mathrm{~N} 2)$, the distribution of slope is broad and peaks at $\sim-0.05 \mathrm{dex}^{-1}$. The magnitude of the systematic metallicity measurement bias is similar for $Z(\mathrm{O} 3 \mathrm{~N} 2)$ and $Z(\mathrm{~N} 2 \mathrm{O} 2)$, but $Z(\mathrm{O} 3 \mathrm{~N} 2)$ in DIG varies much more significantly. Using $Z(\mathrm{O} 3 \mathrm{~N} 2)$ will not bias the measurement if we average a large sample of galaxies, but it will inevitably bias the metallicity in individual galaxies as shown in Section 5.4. The large variation of $Z(\mathrm{O} 3 \mathrm{~N} 2)$ and $Z\left(R_{23}\right)$ in DIG is expected because these two indicators involve ionization parameters. For an $\mathrm{H}_{\text {II }}$ region, this does not matter because the metallicity and ionization parameter are linked to each other. This assumption does not hold for DIG. The variation in the ionization parameter in DIG enters the metallicity measurement. The impact of DIG on using $Z(\mathrm{~N} 2), Z\left(R_{23}\right)$ and $Z(\mathrm{O} 3 \mathrm{~N} 2)$ metallicity gradients is discussed in Sections 5.1, 5.3 and 5.4, respectively. For Z(IZI), the metallicities derived for DIG are systematically lower. Besides, the dispersion of the slope distribution is broadest, with $\sigma \sim 0.3$. This means that IZI not only systematically biases the metallicity of DIG lower, but also introduces large errors into the metallicity measurements.

\subsubsection{Metallicities versus $\Sigma_{\mathrm{H} \alpha}$ relation: Split by Stellar Mass}

In Figs 30 and 31 , we show the normalized $Z$ versus $\Sigma_{\mathrm{H} \alpha}$ relations for galaxies with stellar mass less than $10^{9.43}$ and higher than $10^{10.08}$, respectively. They are the one-third least massive and one-third most massive galaxies in our sample. The $\Delta Z(\mathrm{~N} 2 \mathrm{O} 2), \Delta Z\left(R_{23}\right)$, $\Delta Z(\mathrm{~N} 2 \mathrm{~S} 2 \mathrm{H} \alpha)$ and $\Delta Z(\mathrm{~N} 2)$ versus $\Delta \log \Sigma_{\mathrm{H} \alpha}$ relations are similar in the most massive and least massive galaxies. However, the $\Delta Z(\mathrm{O} 3 \mathrm{~N} 2)$ versus $\Delta \log \Sigma_{\mathrm{H} \alpha}$ relations in the most massive galaxies have positive slopes while those in the least massive galaxies have negative slopes. This is mostly due to the dependence of the 

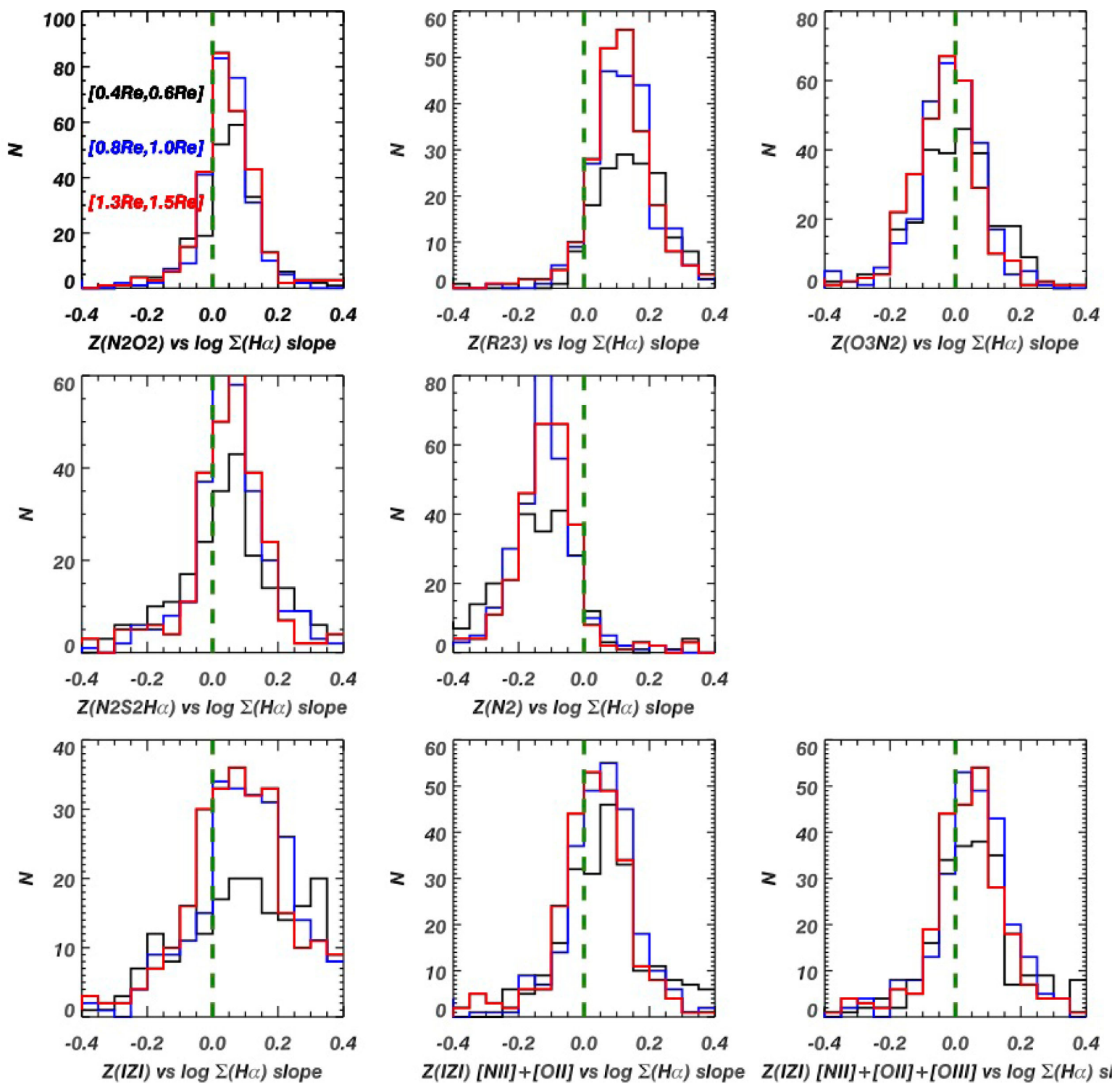

Figure 29. The distribution of metallicities versus $\Sigma(\mathrm{H} \alpha)$ slope at three radii. The black line is for radius within $\left[0.4 R_{\mathrm{e}}, 0.6 R_{\mathrm{e}}\right]$, the blue line is for radius in $\left[0.8 R_{\mathrm{e}}, 1.0 R_{\mathrm{e}}\right]$ and the red line is for the radius in $\left[1.3 R_{\mathrm{e}}, 1.5 R_{\mathrm{e}}\right]$. The green dashed line has slope $=0$ line for reference.The metallicity is independent of surface brightness; the reason we are seeing a dependence of metallicity on $\mathrm{H} \alpha$ surface brightness is due to the contamination by DIG.

$\Delta \log \left[\mathrm{O}_{\mathrm{III}}\right] / \mathrm{H} \beta$ versus $\Delta \log \Sigma_{\mathrm{H} \alpha}$ relation on stellar mass. The dependence of DIGs impact on stellar mass means it is crucial to take care of DIG contamination when comparing the metallicity and metallicity gradient of galaxies of different masses using $Z(O 3 N 2)$.

In summary,

(i) Metallicities derived using N2O2 are optimal because they exhibit the smallest bias and error.

(ii) Metallicities derived using the $\mathrm{O} 3 \mathrm{~N} 2$ or $\mathrm{N} 2 \mathrm{~S} 2 \mathrm{H} \alpha$ (Dopita et al. 2016) for DIG can be significantly higher or lower than those for $\mathrm{H}$ II regions. Using $\mathrm{O} 3 \mathrm{~N} 2$ or $\mathrm{N} 2 \mathrm{~S} 2 \mathrm{H} \alpha$ to derive metallicity can bias the metallicity gradient by $\pm 0.05 R_{\mathrm{e}}^{-1}$ for an individual galaxy if the contamination by DIG is not accounted for. For a large sample of galaxies (Sánchez et al. 2014; Ho et al. 2015), the bias for different galaxies may cancel out when deriving average metallicity gradients.

(iii) $R_{23}$-derived metallicities for DIG are lower than those for $\mathrm{H}$ II regions due to a lower ionization parameter. Using $R_{23}$ to de- rive metallicity will systematically bias, the metallicity gradient by $\sim-0.1 R_{\mathrm{e}}^{-1}$ because of DIG.

(iv) Using $\mathrm{N} 2=[\mathrm{N}$ II] $] / \mathrm{H} \alpha$ to derive metallicities will systematically bias the metallicity gradient by $\sim 0.05-0.1 \mathrm{dex} R_{\mathrm{e}}{ }^{-1}$, considering that DIG typically shows 0.2 dex higher $[\mathrm{N}$ II] $/ \mathrm{H} \alpha$.

(v) IZI works well for the $\mathrm{H}$ II region-dominated regions, but fails in deriving metallicities of DIG, probably because IZI currently only contains $\mathrm{H}$ II region models.

\subsection{Metallicity gradients in the local universe and at high redshift}

For the local universe, we usually have [O II], [O III], $\mathrm{H} \beta$, [N II], $\mathrm{H} \alpha$ and $\left[\mathrm{S}_{\mathrm{II}}\right]$ to derive metallicities. Recent works find that the local starforming galaxies exhibit a universal metallicity gradient of -0.1 dex $R_{\mathrm{e}}^{-1}$ within $2 R_{\mathrm{e}}$ (e.g. Sánchez et al. 2014; Ho et al. 2015). These works employ O3N2 (Sánchez et al. 2014; Ho et al. 2015) and N2O2 (Ho et al. 2015) to derive the metallicity. Sánchez et al. 

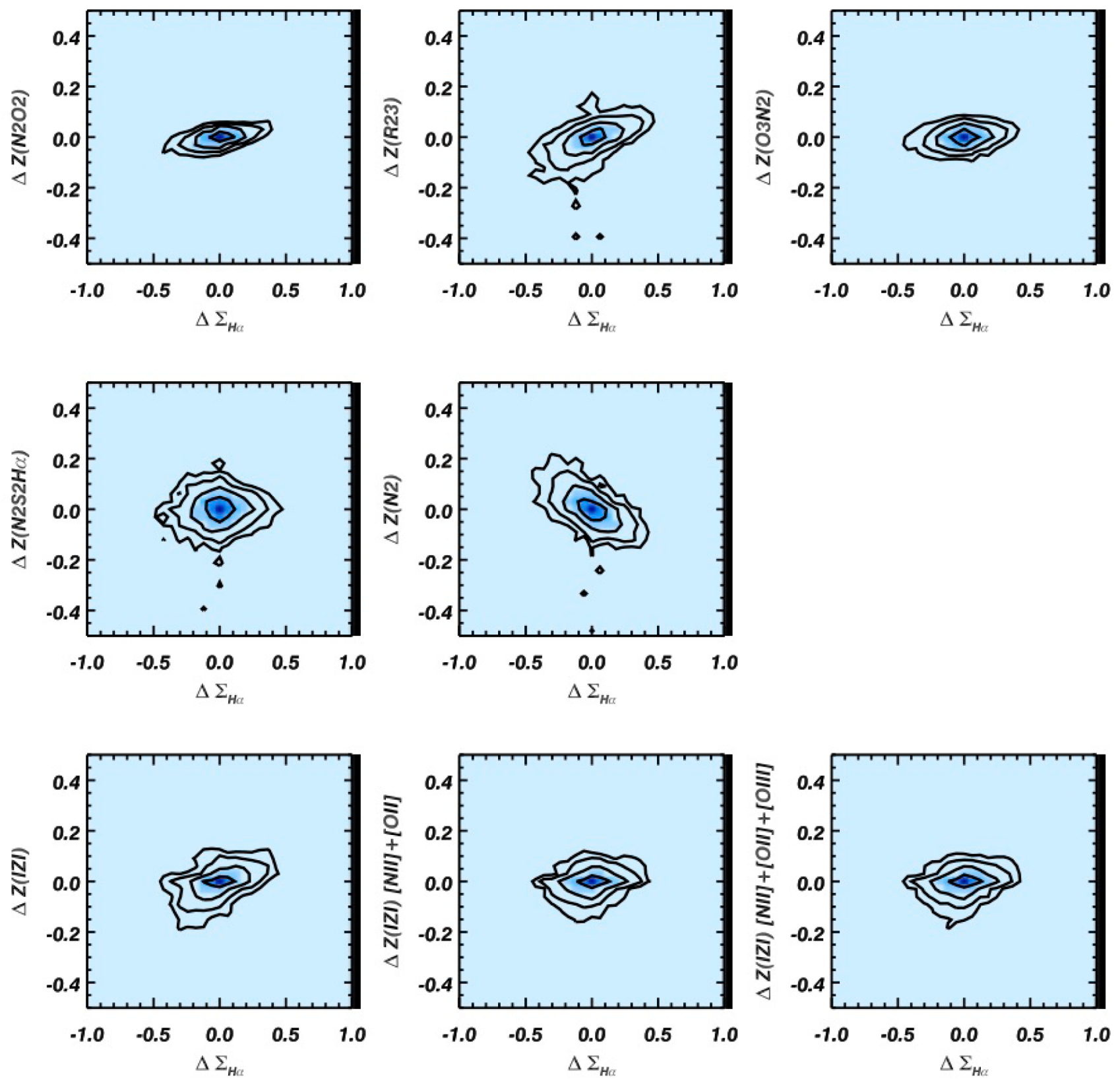

Figure 30. $\Delta \mathrm{Z}$ versus $\Delta \log \Sigma_{\mathrm{H} \alpha}$ relation at $\left[0.4 R_{\mathrm{e}}, 0.6 R_{\mathrm{e}}\right]$ for the one-third most massive galaxies in our sample. We normalize the $\mathrm{Z}$ versus log $\Sigma_{\mathrm{H} \alpha}$ relation by subtracting the median $\mathrm{Z}$ and median $\log \Sigma_{\mathrm{H} \alpha}$. All the spaxels in a galaxy are weighted by $\frac{1}{N}$, where $N$ is the number of valid spaxels in the galaxy.

(2014) isolated $\mathrm{H}$ II regions from DIG and used O3N2 to derive the metallicities using $\mathrm{H}$ II regions. The contamination of DIG is small. Ho et al. (2015) rejected the DIG-dominated spaxels by imposing $\mathrm{S} / \mathrm{N}>3$, and $Z(\mathrm{O} 3 \mathrm{~N} 2)$ and $Z(\mathrm{~N} 2 \mathrm{O} 2)$ give consistent metallicities and metallicity gradients. The metallicity gradient at $R<2 R_{\mathrm{e}}$ is robust against DIG contamination. Using MaNGA data and the robust metallicity estimator $\mathrm{N} 2 \mathrm{O} 2$ to remeasure metallicities would be worthwhile to re-examine the metallicity gradient at large radius.

At high redshift, we need a new calibration of strong-line metallicity indicators because the physical properties are different in highredshift galaxies compared to local galaxies. High-redshift starforming galaxies are systematically offset towards higher $[\mathrm{O}$ III $] / \mathrm{H} \beta$ on the BPT diagram relative to the local star-forming galaxy locus
(Shapley et al. 2005; Erb et al. 2006; Brinchmann, Pettini \& Charlot 2008; Liu et al. 2008; Hainline et al. 2009; Wright et al. 2010; Trump et al. 2011; Kewley et al. 2013; Steidel et al. 2014). The line ratio shifts of high-redshift star-forming galaxies are similar to DIG. Based on photoionization models, Steidel et al. (2014) concluded that the offset on the BPT diagram of the $z \sim 2.3$ star-forming galaxies is caused by a combination of harder stellar ionizing radiation field, higher ionization parameter and higher $\mathrm{N} / \mathrm{O}$ at a given $\mathrm{O} / \mathrm{H}$ compared to most local galaxies. A new calibration between $\mathrm{N} 2$ and $\mathrm{O} 3 \mathrm{~N} 2$ was obtained. A higher N/O abundance has been claimed to explain the offset in the BPT Diagram (Masters et al. 2014; Shapley et al. 2015; Sanders et al. 2016). There is debate whether the metallicity gradients at high redshift are flatter or steeper 

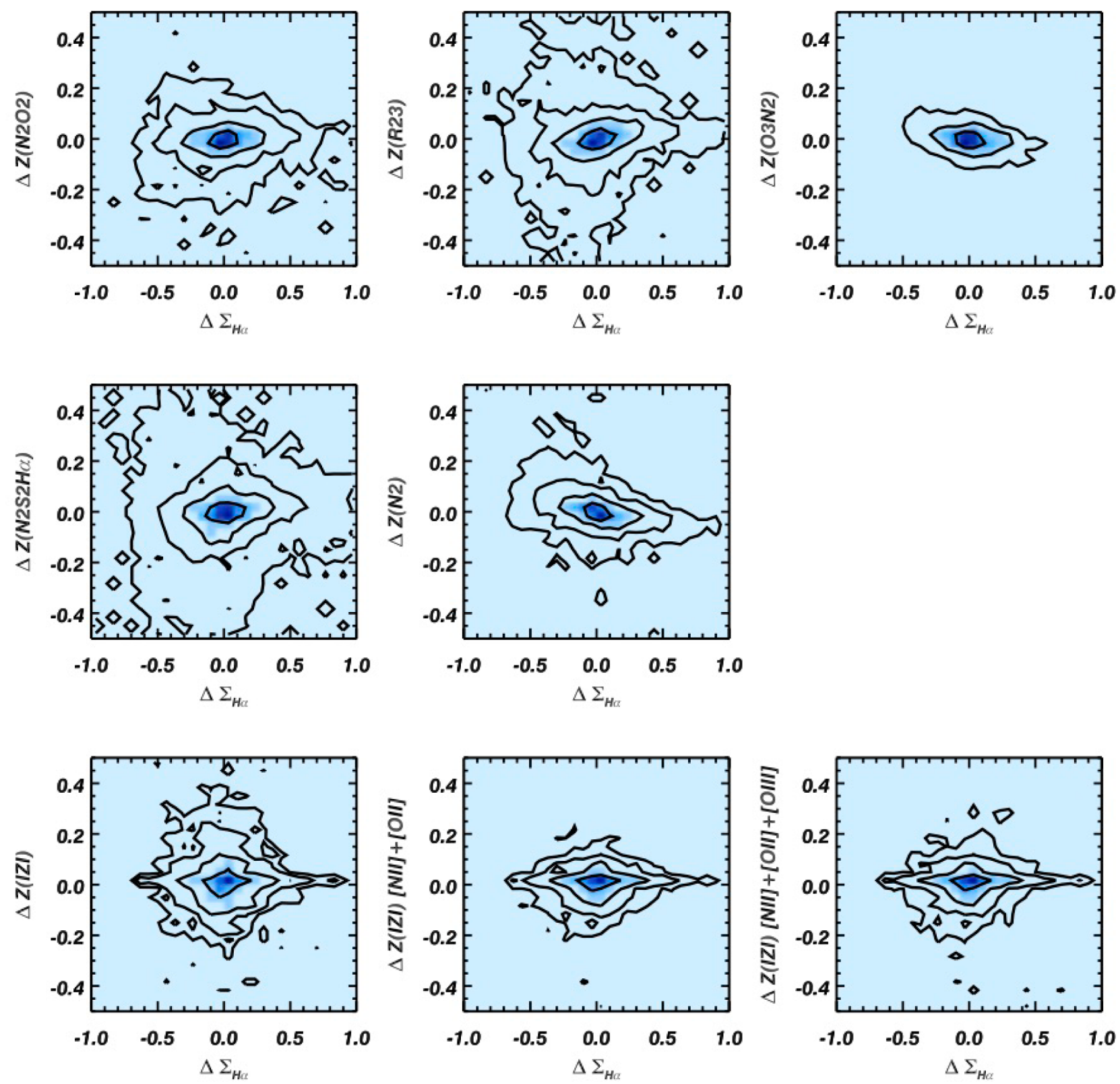

Figure 31. $\Delta Z$ versus $\Delta \log \Sigma_{\mathrm{H} \alpha}$ relation at $\left[0.4 R_{\mathrm{e}}, 0.6 R_{\mathrm{e}}\right]$ for the one-third least massive galaxies in our sample. We normalize the $Z$ versus log $\Sigma_{\mathrm{H} \alpha}$ relation by subtracting the median $Z$ and median $\log \Sigma_{\mathrm{H} \alpha}$. All the spaxels in a galaxy are weighted by $\frac{1}{N}$, where $N$ is the number of valid spaxels in the galaxy.

(Yuan et al. 2011; Swinbank et al. 2012; Jones et al. 2013, 2015; Leethochawalit et al. 2016; Wuyts et al. 2016). It takes time and resources to obtain all $[\mathrm{O} \mathrm{III}], \mathrm{H} \beta,[\mathrm{N} \mathrm{II}], \mathrm{H} \alpha$ for a galaxy at high redshift. In most cases, either $[\mathrm{O} \mathrm{III}]+\mathrm{H} \beta$ or $[\mathrm{N}$ II] $+\mathrm{H} \alpha$ is observed. The few lines available limit the metallicity estimator to either $R_{23}$ or N2, two indicators that are impacted most by the presence of DIG. This makes the metallicity measurements at high- $z$ vulnerable to the contamination of DIG. However, we note that current studies are unlikely to be seriously affected by DIG because in general, they are forming stars at an incredibly high rate and they are very well represented by pure $\mathrm{H}_{\mathrm{II}}$ region spectra. Future studies at high redshift probing low-surface brightness limits (e.g. with JWST) may have to take into account the contribution of DIG. One needs to be cautious when making comparisons of high- $z$ and low- $z$ line ratios because DIG matters more at low-z.

\section{OTHER RELEVANT ISSUES}

\subsection{Line ratios versus $H \alpha$ surface brightness relation} for an individual $\mathrm{H}$ II region

Relaño et al. (2010) studied the line ratios and $\mathrm{H} \alpha$ surface brightness of NGC 595, one of the most luminous $\mathrm{H}_{\text {II }}$ regions in M33. The scale of NGC 595 is about 300 pc. By comparing the $\mathrm{H} \alpha$ surface brightness distribution shown in their fig. 3 and the $[\mathrm{N} \mathrm{II}] / \mathrm{H} \alpha$, $\left[\mathrm{S}_{\mathrm{II}}\right] / \mathrm{H} \alpha$ and $\left[\mathrm{O}_{\mathrm{III}}\right] / \mathrm{H} \beta$ maps in Fig. 6 , we can see that the lowsurface brightness regions, which are located far away from the ionizing stars, have high $\left[\mathrm{N}_{\mathrm{II}}\right] / \mathrm{H} \alpha,\left[\mathrm{S}_{\mathrm{II}}\right] / \mathrm{H} \alpha$ and lower $\left[\mathrm{O}_{\mathrm{III}}\right] / \mathrm{H} \beta$. The line ratios versus $\mathrm{H} \alpha$ surface brightness relations are derived on a scale of hundreds of parsecs around an individual $\mathrm{H}$ II region. Madsen et al. (2006) saw the same trend in their observation of Milky Way. The resolution of the WHAM survey is $1^{\circ}$, which 
corresponds to $90 \mathrm{pc}$ at $5 \mathrm{kpc}$. So the physical scale of WHAM is tens to hundred parsecs. Do these local relations on scales of hundreds of parsecs extend to kiloparsec scale probed by MaNGA? MaNGA's spatial resolution of $1 \mathrm{kpc}$ means that each PSF may include tens or even hundreds of $\mathrm{H}$ II regions. If the $\mathrm{H} \alpha$ surface brightness versus line ratios relation is confined to 300 pc scales, when smeared by a kiloparsec-scale PSF, the line ratio variation will be smoothed out and disappear. In other words, if DIG is dominated by regions within $10 \mathrm{pc}$ of each $\mathrm{H}_{\mathrm{II}}$ region, then we would not see the line ratios versus $\Sigma_{\mathrm{H} \alpha}$ relation after smoothing it with a kiloparsec-sized PSF. The smearing effect can produce a surface brightness gradient but cannot produce an anticorrelation between surface brightness and line ratios. The fact that we see a surface brightness versus line ratio relation on kiloparsec scales means that these relations for individual $\mathrm{H}$ II region cannot be local, but extend to kiloparsec scales. The DIG we see is dominated by regions far away (kpc) from individual $\mathrm{H}$ II regions. In edge-on galaxies, we do see that DIG can extend to a few kiloparsecs above the galaxy plane (e.g. Rand 1996, 1997; Rossa \& Dettmar 2000; Kehrig et al. 2012; Jones et al. 2016).

Then what does the line ratio versus $\mathrm{H} \alpha$ surface brightness relation tell us? What we need to keep in mind is that we are looking at the average properties of the galaxy at our resolution. As discussed in detail in Appendix A, this relation is preserved for individual $\mathrm{H}$ II regions no matter what resolution we use to observe the galaxy. However, under poor resolution, many $\mathrm{H}$ II regions are mixed and their relations are also mixed. We do not know how many $\mathrm{H}$ II regions there are in one spaxel and what their line ratio versus $\mathrm{H} \alpha$ surface brightness relations looks like. What we see is the average properties within $1 \mathrm{kpc}$, and how the line ratios and surface brightness change coherently on this scale. The curvature of the relation does tell us that an $\mathrm{H}$ II region is not a simple Strömgren sphere but includes an extra component of DIG which has different line ratios. At $1 \mathrm{kpc}$ scale, we can model the ISM as a combination of DIG and $\mathrm{H}$ II regions with their respective line ratios. This relation also illustrates the feasibility of using $\mathrm{H} \alpha$ surface brightness to roughly separate DIG and $\mathrm{H}$ II regions.

\subsection{Shedding light on composite region and LI(N)ER}

Any factors that change the line ratios can influence the positions on the diagnostic diagrams (e.g. Zhang et al. 2008; Pèrez-Montero $\&$ Contini 2009). In Figs 15 and 16, we explored the distribution of each spaxel on the BPT diagram: $[\mathrm{O}$ III] $/ \mathrm{H} \beta$ versus $[\mathrm{N}$ II] $/ \mathrm{H} \alpha$ and $[\mathrm{O}$ III $] / \mathrm{H} \beta$ versus $[\mathrm{S}$ II] $/ \mathrm{H} \alpha$ (Baldwin et al. 1981; Veilleux \& Osterbrock 1987). In the $\left[\mathrm{O}_{\mathrm{III}}\right] / \mathrm{H} \beta$ versus $\left[\mathrm{N}_{\mathrm{II}}\right] / \mathrm{H} \alpha$ diagram, we plot the demarcations from Kewley et al. (2001) and Kauffmann et al. (2003) to identify the ionizing source properties. We colour-code the dots by $\mathrm{H} \alpha$ surface brightness, and the dots in composite and $\mathrm{LI}(\mathrm{N}) \mathrm{ER}$ region have low $\mathrm{H} \alpha$ surface brightness. As discussed in Section 4, a hardened O star spectrum filtered by ISM cannot produce LI(N)ER-like emissions, while hot evolved stars like pAGB stars can produce the LI(N)ER-like line ratios. In Belfiore et al. (2016a), they classified galaxies that show LI(N)ER-like emission into two classes: cLIER and eLIER. cLIER shows LI(N)ER-like emission in the centre, while eLIER shows extended LI(N)ER-like emission all over the galaxy. In some galaxies in our sample, the line ratios could extend to LI(N)ER regions on the BPT diagrams (see also Galarza et al. 1999; Kaplan et al. 2016). We suspect that these two phenomenon may have a common origin. However, the galaxies that show eLIER emission and cLIER could have different stellar population and gas metallicity from the sample we study in this pa- per. Therefore, a lot more work is needed to prove if they are really the same. We defer this to a future paper. The Shocked POststarburst Galaxy Survey (SPOGS; Alatalo et al. 2016a,b) explores a sample of galaxies selected from SDSS Data Release 7 that show LI(N)ER like emission-line ratios and post-starburst spectral features. A similar phenomenon is found by Yan et al. (2006). After OB stars die, the spectrum of the galaxy is dominated by spectral features of $\mathrm{A}$ stars, characterizing the post-starburst signature. According to our grids, the decrease in OB stars and increase LI(N)ER-like emission line ratios are naturally linked through increasing photoionization by evolved stars. DIG, cLIER and SPOGS are very similar to each other and the study of their similarity and distinctions can greatly help us understand the ionized gas in different types of galaxies. From our figures, DIG-dominated regions can mimic composite or even AGN spectra. If the selection of AGN is purely based on the BPT diagram, star-forming galaxies with significant DIG could be incorrectly classified as AGN. DIG and AGNs might be distinguished using the WHAN diagram (Cid Fernandes et al. 2010, 2011) due to their different $\mathrm{EW}(\mathrm{H} \alpha)$ and $\left[\mathrm{N}_{\mathrm{II}}\right] / \mathrm{H} \alpha$ distributions. This will be explored further in the future. Our analysis demonstrates the importance of considering the presence of DIG while making optical line ratio classifications. Additionally, the analysis illustrates the complexity caused by DIG in interpreting the line ratio diagnostic diagrams. The so-called composite galaxies or LI(N)ERs could be coming from a variety of objects.

Shocks could also produce LI(N)ER-like line ratios. Shocked gas with velocities less than $500 \mathrm{~km} \mathrm{~s}^{-1}$ occupy the $\mathrm{LI}(\mathrm{N}) \mathrm{ER}$ part of the diagnostic diagrams (Farage et al. 2010; Rich, Kewley \& Dopita 2011), while shocked gas with velocities greater than $500 \mathrm{~km} \mathrm{~s}^{-1}$ fall in the Seyfert part of the diagram (Allen et al. 2008; Kewley et al. 2013). Shock model can produce the enhanced $\left[\mathrm{S} \mathrm{II}_{\mathrm{II}}\right] / \mathrm{H} \alpha,\left[\mathrm{N}\right.$ II] $/ \mathrm{H} \alpha,\left[\mathrm{O}_{\mathrm{II}}\right] / \mathrm{H} \beta,\left[\mathrm{O}_{\mathrm{I}}\right] / \mathrm{H} \alpha$ and $[\mathrm{O}$ III] $] / \mathrm{H} \beta$ we see. Additionally, the temperature of shocked gas is elevated (Allen et al. 2008). To test if shocks are indeed a major ionization source for DIG, high-spectral resolution spectra $(\sim 4000)$ are needed to separate different velocity components and constrain the kinematics. Our spectral resolution $(R \sim 2000)$ limits our ability to test for shocks. We leave the question of the full nature of ionization sources of DIG for further studies.

\subsection{How to minimize the impact of DIG}

The best way to minimize the impact of DIG is to separate DIG and $\mathrm{H}$ II regions spatially. With high-spatial resolution IFS data like CALIFA, it is possible to resolve individual $\mathrm{H}$ in regions to lower the contamination of DIG. For low-spatial resolution data like MaNGA and SAMI, the individual spaxels are covering kiloparsec-scale regions, thus the emission is a mix of $\mathrm{H}_{\mathrm{II}}$ regions and DIG. The mixing also happens for MUSE data of high-redshift galaxies. For these data, selecting high $\Sigma_{\mathrm{H} \alpha}$ spaxels would mitigate the impact of DIG. As we have shown in this paper, the high $\Sigma_{\mathrm{H} \alpha}$ regions have $\mathrm{H}$ II region line ratios. Besides, the metallicity gradient derived using only the high $\Sigma_{\mathrm{H} \alpha}$ regions for different strong line method: $Z(\mathrm{~N} 2 \mathrm{O} 2)$, $Z\left(R_{23}\right), Z(\mathrm{O} 3 \mathrm{~N} 2), Z(\mathrm{~N} 2 \mathrm{~S} 2 \mathrm{H} \alpha)$ are consistent with each other. This means the high $\Sigma_{\mathrm{H} \alpha}$ regions, even though contaminated by DIG due to beam smearing, are $\mathrm{H}$ II region-dominated. A $\Sigma_{\mathrm{H} \alpha}$ cut is a robust and easy way to reduce the impact of DIG. The exact value of $\Sigma_{\mathrm{H} \alpha}$ cut depends on the spatial resolution. For our MaNGA survey, $\Sigma_{\mathrm{H} \alpha}>39 \mathrm{erg} \mathrm{s}^{-1} \mathrm{kpc}^{-2}$ select reliable $\mathrm{H}$ II region-dominated spaxels. An equivalent width (EW) cut is not recommended since EW depends on metallicity (e.g. Tresse et al. 1999). For low-metallicity regions, for example, in low-metallicity galaxies or the outskirt of 
a galaxy, EW will be high due to the low metallicity. A EW cut suitable for the centre of a galaxy will select DIG-contaminated spaxels at the outskirt of this galaxy. So selecting high $\Sigma_{\mathrm{H} \alpha}$ regions is a reliable and convenient way to minimize DIG contamination.

\section{SUMMARY AND CONCLUSIONS}

We selected a sample of 365 blue face-on galaxies from 1391 galaxies observed by MaNGA, and illustrated the impact of DIG on line ratios, interpretation of diagnostic diagrams and metallicity measurements. We find that $\mathrm{H} \alpha$ surface brightness is a good indicator to separate $\mathrm{H}$ II regions from DIG. DIG shows distinct properties as listed below.

(i) $\left.\left[\mathrm{S}_{\mathrm{II}}\right] / \mathrm{H} \alpha, \mathrm{N}_{\mathrm{II}}\right] / \mathrm{H} \alpha,\left[\mathrm{O}_{\mathrm{II}}\right] / \mathrm{H} \beta$, and $\left[\mathrm{O}_{\mathrm{I}}\right] / \mathrm{H} \alpha$ are enhanced in DIG relative to $\mathrm{H}$ II regions.

(ii) DIG has lower $[\mathrm{O} \mathrm{III}] /[\mathrm{O} \mathrm{II}]$, indicating lower ionization parameter. $\left[\mathrm{O}\right.$ III] $/ \mathrm{H} \beta$ of DIG can be higher or lower than $\mathrm{H}_{\text {II }}$ regions.

(iii) On BPT diagrams, contamination by DIG moves $\mathrm{H}$ II regions towards composite or LI(N)ER-like regions. A harder ionizing spectrum is needed to explain DIG line ratios.

(iv) Leaky $\mathrm{H}$ II region models only shift the line ratios slightly relative to $\mathrm{H}$ II region models, thus fail to explain composite/LI(N)ER line ratios displayed by DIG. Leaky $\mathrm{H}$ II region models cannot explain the $[\mathrm{O}$ III $] / \mathrm{H} \beta$ but do pretty well for the other line ratios.

(v) Our result favours ionization by evolved stars as a major ionization source for DIG with LI(N)ER-like emission.

(vi) Metallicities derived using $\mathrm{N} 2 \mathrm{O} 2=[\mathrm{N}$ II]/[O II $]$ are optimal because they exhibit the smallest bias and scatter.

(vii) Metallicities derived using the $\mathrm{O} 3 \mathrm{~N} 2=$ $([\mathrm{O} I I I] / \mathrm{H} \beta) /([\mathrm{N} \mathrm{II}] / \mathrm{H} \alpha)$ or $\mathrm{N} 2 \mathrm{~S} 2 \mathrm{H} \alpha=8.77+\log \left[\mathrm{N}_{\mathrm{II}}\right] /\left[\mathrm{S}_{\mathrm{II}}\right]$ $+0.264 \times \log \left[\mathrm{N}_{\mathrm{II}}\right] / \mathrm{H} \alpha$ (Dopita et al. 2016) for DIG can be significantly higher or lower than those for $\mathrm{H}$ II regions. Using $\mathrm{O} 3 \mathrm{~N} 2$ or $\mathrm{N} 2 \mathrm{~S} 2 \mathrm{H} \alpha$ to derive metallicities can bias the metallicity gradient by $\pm 0.05 \mathrm{dex} R_{\mathrm{e}}^{-1}$ for an individual galaxy if the contamination by DIG is not accounted for. $R_{23}$-derived metallicities for DIG are lower than those for $\mathrm{H}_{\mathrm{II}}$ regions due to a lower ionization parameter. Using $R_{23}$ to derive metallicities will systematically bias the metallicity gradient by $\sim-0.1$ dex $R_{\mathrm{e}}^{-1}$ because of DIG. Using $\mathrm{N} 2=[\mathrm{N} I] / \mathrm{H} \alpha$ to derive metallicities will systematically bias the metallicity gradient by $\sim 0.05-0.1 \mathrm{dex} R_{\mathrm{e}}{ }^{-1}$, considering that DIG typically shows 0.2 dex higher $[\mathrm{N}$ II] $/ \mathrm{H} \alpha$.

(viii) The metallicities in high-redshift galaxies are mostly derived using $R_{23}$ or $\mathrm{N} 2$, rendering their metallicity and metallicity gradient measurements most vulnerable to the impact of DIG. Using $\mathrm{Z}(\mathrm{N} 2 \mathrm{~S} 2 \mathrm{H} \alpha)$ for high-redshift galaxies is more robust to prevent the contamination by DIG. For most of the recent high- $z$ observations, the contamination by DIG is probably not severe because we only see high $\Sigma_{\mathrm{H} \alpha}$ regions. When comparing the metallicities of high- $z$ galaxies with local galaxies, one needs to use caution since DIG might impact metallicity measurements of local galaxies.

\section{ACKNOWLEDGEMENTS}

$\mathrm{KB}$ is supported by World Premier International Research Center Initiative (WPI Initiative), MEXT, Japan and by JSPS KAKENHI Grant Number 15K17603. MAB acknowledges support from NSF AST-1517006. RM acknowledge support by the Science and Technology Facilities Council (STFC) and the ERC Advanced Grant 695671 'QUENCH'. C.A.T. acknowledges support from National Science Foundation of the United States grant no. 1412287. DB was supported by grant RSF 14-50-00043. AD acknowledges support from The Grainger Foundation. Funding for the Sloan Digital Sky
Survey IV has been provided by the Alfred P. Sloan Foundation, the US Department of Energy Office of Science and the Participating Institutions. SDSS-IV acknowledges support and resources from the Center for High-Performance Computing at the University of Utah. The SDSS web site is www.sdss.org. SDSS-IV is managed by the Astrophysical Research Consortium for the Participating Institutions of the SDSS Collaboration including the Brazilian Participation Group, the Carnegie Institution for Science, Carnegie Mellon University, the Chilean Participation Group, the French Participation Group, Harvard-Smithsonian Center for Astrophysics, Instituto de Astrofísica de Canarias, The Johns Hopkins University, Kavli Institute for the Physics and Mathematics of the Universe (IPMU)/ University of Tokyo, Lawrence Berkeley National Laboratory, Leibniz Institut für Astrophysik Potsdam (AIP), Max-Planck-Institut für Astronomie (MPIA Heidelberg), Max-Planck-Institut für Astrophysik (MPA Garching), Max-Planck-Institut für Extraterrestrische Physik (MPE), National Astronomical Observatory of China, New Mexico State University, New York University, University of Notre Dame, Observatrio Nacional/ MCTI, The Ohio State University, Pennsylvania State University, Shanghai Astronomical Observatory, United Kingdom Participation Group, Universidad Nacional Autónoma de México, University of Arizona, University of Colorado Boulder, University of Oxford, University of Portsmouth, University of Utah, University of Virginia, University of Washington, University of Wisconsin, Vanderbilt University and Yale University.

\section{REFERENCES}

Alatalo K. et al., 2016a, ApJS, 224, 38

Alatalo K. et al., 2016b, ApJ, 827, 016

Allen M. G., Groves B. A., Dopita M. A., Sutherland R. S., Kewley L. J., 2008, ApJS, 178, 20

Alloin D., Collin-Souffrin S., Joly M., Vigroux L., 1979, A\&A, 78, 200

Andrews B. H., Martini P., 2013, ApJ, 765, 140

Baldwin J. A., Phillips M. M., Terlevich R., 1981, PASP, 93, 5

Baldwin J. A., Ferland G. J., Martin P. G., Corbin M. R., Cota S. A., Peterson B. M., Slettebak A., 1991, ApJ, 374, 580

Belfiore F. et al., 2015, MNRAS, 449, 867

Belfiore F. et al., 2016a, MNRAS, preprint (arXiv:1609.01737)

Belfiore F. et al., 2016b, MNRAS, 461, 3111

Binette L., Magris C. G., Stasińska G., Bruzual A. G., 1994, A\&A, 292, 13

Blanc G. A., Heiderman A., Gebhardt K., Evans N. J., II, Adams J., 2009, ApJ, 704, 842-862

Blanc G. A., Kewley L., Vogt F. P. A., Dopita M. A., 2015, ApJ, 798, 99

Brinchmann J., Pettini M., Charlot S., 2008, MNRAS, 385, 769

Bruzual G., Charlot S., 2003, MNRAS, 344, 1000

Bundy K. et al., 2015, ApJ, 798, 7

Cid Fernandes R., Stasińska G., Schlickmann M. S., Mateus A., Vale Asari N., Schoenell W., Sodré L., 2010, MNRAS, 403, 1036

Cid Fernandes R., Stasińska G., Mateus A., Vale Asari N., 2011, MNRAS 413, 1687

Collins J. A., Rand R. J., 2001, ApJ, 551, 57

Denicoló G., Terlevich R., Terlevich E., 2002, MNRAS, 330, 69

Dettmar R.-J., 1990, A\&A, 232, L15

Dopita M. A., Kewley L. J., Heisler C. A., Sutherland R. S., 2000, ApJ, 542, 224

Dopita M. A. et al., 2006, ApJs, 167, 177

Dopita M. A., Sutherland R. S., Nicholls D. C., Kewley L. J., Vogt F. P. A., 2013, ApJs, 208, 10

Dopita M. A., Kewley L. J., Sutherland R. S., Nicholls D. C., 2016, ApJS, 361,61

Drory N. et al., 2015, AJ, 149, 77

Erb D. K., Shapley A. E., Pettini M., Steidel C. C., Reddy N. A., Adelberger K. L., 2006, ApJ, 644, 813 
Farage C. L., McGregor P. J., Dopita M. A., Bicknell G. V., 2010, ApJ, 724, 267

Ferguson A. M. N., Wyse R. F. G., Gallagher J. S., III, Hunter D. A., 1996, AJ, 111, 2265

Ferland G. J., Korista K. T., Verner D. A., Ferguson J. W., Kingdon J. B., Verner E. M., 1998, PASP, 110, 761

Fitzpatrick E. L., 1999, PASP, 111, 63

Flores-Fajardo N., Morisset C., Stasińska G., Binette L., 2011, MNRAS, 415,2182

Galarza V. C., Walterbos R. A. M., Braun R., 1999, AJ, 118, 2775

Garay G., Lizano S., 1999, PASP, 111, 1049

Garnett D. R., 2002, ApJ, 581, 1019

Giammanco C., Beckman J. E., Cedrés B., 2005, A\&A, 438, 599

Gomes J. M. et al., 2016, A\&A, 588, A68

Greenawalt B., Walterbos R. A. M., Braun R., 1997, ApJ, 483, 666

Greenawalt B., Walterbos R. A. M., Thilker D., Hoopes C. G., 1998, ApJ, 506,135

Gunn J. E. et al., 2006, AJ, 131, 2332

Haffner L. M., Reynolds R. J., Tufte S. L., 1999, ApJ, 523, 223

Haffner L. M. et al., 2009, Rev. Mod. Phys., 81, 969

Hainline K. N., Shapley A. E., Kornei K. A., Pettini M., Buckley-Geer E., Allam S. S., Tucker D. L., 2009, ApJ, 701, 52

Hausen N. R., Reynolds R. J., Haffner L. M., 2002, AJ, 124, 3336

Ho I.-T., Kudritzki R.-P., Kewley L. J., Zahid H. J., Dopita M. A., Bresolin F., Rupke D. S. N., 2015, MNRAS, 448, 2030

Hoopes C. G., Walterbos R. A. M., 2003, ApJ, 586, 902

Hoopes C. G., Walterbos R. A. M., Greenwalt B. E., 1996, AJ, 112, 1429

Hoopes C. G., Walterbos R. A. M., Rand R. J., 1999, ApJ, 522, 669

Hunt L. K., Hirashita H., 2009, A\&A, 507, 1327

Jones T., Ellis R. S., Richard J., Jullo E., 2013, ApJ, 765, 48

Jones T. et al., 2015, AJ, 149, 107

Jones A. et al., 2016, A\&A, preprint (arXiv:1612.03920)

Kaplan K. F. et al., 2016, MNRAS, 462, 1642

Kauffmann G. et al., 2003, MNRAS, 346, 1055

Kehrig C. et al., 2012, A\&A, 540, A11

Kennicutt R. C., Jr, 1984, ApJ, 287, 116

Kewley L. J., Dopita M. A., 2002, ApJs, 142, 35

Kewley L. J., Ellison S. L., 2008, ApJ, 681, 1183

Kewley L. J., Heisler C. A., Dopita M. A., Lumsden S., 2001, ApJS, 132, 37

Kewley L. J., Groves B., Kauffmann G., Heckman T., 2006, MNRAS, 372, 961

Kewley L. J., Dopita M. A., Leitherer C., Davé R., Yuan T., Allen M., Groves B., Sutherland R., 2013, ApJ, 774, 100

Kim K.-T., Koo B.-C., 2001, ApJ, 549, 979

Kobulnicky H. A., Kewley L. J., 2004, ApJ, 617, 240

Kreckel K., Blanc G. A., Schinnerer E., Groves B., Adamo A., Hughes A., Meidt S., 2016, ApJ, 827, 103

Lanz T., Hubeny I., 2003, ApJS, 146, 417

Law D. R. et al., 2015, AJ, 150, 19

Law D. R. et al., 2016, AJ, 152, 83

Lee H., Skillman E. D., Cannon J. M., Jackson D. C., Gehrz R. D., Polomski E. F., Woodward C. E., 2006, ApJ, 647, 970

Leethochawalit N., Jones T. A., Ellis R. S., Stark D. P., Richard J., Zitrin A., Auger M., 2016, ApJ, 820, 84

Lequeux J., Peimbert M., Rayo J. F., Serrano A., Torres-Peimbert S., 1979, A\&A, 80, 155

Levesque E. M., Kewley L. J., Larson K. L., 2010, AJ, 139, 712

Li C. et al., 2015, ApJ, 804, 125

Liu X., Shapley A. E., Coil A. L., Brinchmann J., Ma C.-P., 2008, ApJ, 678, 758

McClure R. D., van den Bergh S., 1968, AJ, 73, 313

McGaugh S. S., 1991, ApJ, 380, 140

Madsen G. J., Reynolds R. J., Haffner L. M., 2006, ApJ, 652, 401

Maiolino R. et al., 2008, A\&A, 488, 463

Mannucci F. et al., 2009, MNRAS, 398, 1915

Mannucci F., Cresci G., Maiolino R., Marconi A., Gnerucci A., 2010, MNRAS, 408, 2115
Markwardt C. B., 2009, in Bohlender D. A., Durand D., Dowler P., eds, ASP Conf. Ser. Vol. 411, Astronomical Data Analysis Software and Systems XVIII. Astron. Soc. Pac., San Francisco, p. 251

Mast D. et al., 2014, A\&A, 561, A129

Masters D. et al., 2014, ApJ, 785, 153

Mierkiewicz E. J., Reynolds R. J., Roesler F. L., Harlander J. M., Jaehnig K. P., 2006, ApJ, 650, L63

Monnet G., 1971, A\&A, 12, 379

Moran S. M. et al., 2012, ApJ, 745, 66

Oey M. S. et al., 2007, ApJ, 661, 801

Osterbrock D. E., Tran H. D., Veilleux S., 1992, ApJ, 389, 305

Otte B., 2001, PhD Thesis, 3233

Otte B., Reynolds R. J., Gallagher J. S., III, Ferguson A. M. N., 2001, ApJ, 560,207

Otte B., Gallagher J. S., III, Reynolds R. J., 2002, ApJ, 572, 823

Pérez-Montero E., 2014, MNRAS, 441, 2663

Pérez-Montero E., Contini T., 2009, MNRAS, 398, 949

Pérez-Montero E. et al., 2013, A\&A, 549, A25

Pérez-Montero E. et al., 2016, A\&A, 595, A62

Pettini M., Pagel B. E. J., 2004, MNRAS, 348, L59

Pilyugin L. S., 2001, A\&A, 369, 594

Pilyugin L. S., Thuan T. X., 2005, ApJ, 631, 231

Rand R. J., 1996, ApJ, 462, 712

Rand R. J., 1997, ApJ, 474, 129

Rand R. J., 1998, ApJ, 501, 137

Rand R. J., 2000, ApJ, 537, L13

Rand R. J., Kulkarni S. R., Hester J. J., 1990, ApJ, 352, L1

Relaño M., Monreal-Ibero A., Vílchez J. M., Kennicutt R. C., 2010, MNRAS, 402, 1635

Reynolds R. J., 1984, ApJ, 282, 191

Reynolds R. J., 1985a, ApJ, 294, 256

Reynolds R. J., 1985b, ApJ, 298, L27

Reynolds R. J., 1990, ApJ, 349, L17

Reynolds R. J., 1991, ApJ, 372, L17

Reynolds R. J., Cox D. P., 1992, ApJ, 400, L33

Reynolds R. J., Hausen N. R., Tufte S. L., Haffner L. M., 1998, ApJ, 494, L99

Rich J. A., Kewley L. J., Dopita M. A., 2011, ApJ, 734, 87

Rossa J., Dettmar R.-J., 2000, A\&A, 359, 433

Rossa J., Dettmar R.-J., 2003a, A\&A, 406, 493

Rossa J., Dettmar R.-J., 2003b, A\&A, 406, 505

Rubin R. H., Simpson J. P., Haas M. R., Erickson E. F., 1991, ApJ, 374, 564

Rubin R. H., Dufour R. J., Walter D. K., 1993, ApJ, 413, 242

Sánchez S. F. et al., 2012, A\&A, 546, A2

Sánchez S. F. et al., 2014, A\&A, 563, A49

Sanders R. L. et al., 2016, ApJ, 816, 23

Sarzi M. et al., 2006, MNRAS, 366, 1151

Shapley A. E., Coil A. L., Ma C.-P., Bundy K., 2005, ApJ, 635, 1006

Shapley A. E. et al., 2015, ApJ, 801, 88

Singh R. et al., 2013, A\&A, 558, A43

Smee S. A. et al., 2013, AJ, 146, 32

Stasińska G. et al., 2008, MNRAS, 391, L29

Steidel C. C. et al., 2014, ApJ, 795, 165

Storchi-Bergmann T., Calzetti D., Kinney A. L., 1994, ApJ, 429, 572

Swinbank A. M., Sobral D., Smail I., Geach J. E., Best P. N., McCarthy I. G., Crain R. A., Theuns T., 2012, MNRAS, 426, 935

Thilker D. A., Walterbos R. A. M., Braun R., Hoopes C. G., 2002, AJ, 124, 3118

Tremonti C. A. et al., 2004, ApJ, 613, 898

Tresse L., Maddox S., Loveday J., Singleton C., 1999, MNRAS, 310, 262

Trump J. R. et al., 2011, ApJ, 743, 144

Tüllmann R., Dettmar R.-J., 2000, A\&A, 362, 119

Vale Asari N., Stasińska G., Morisset C., Cid Fernandes R., 2016, MNRAS, 460,1739

van Zee L., Salzer J. J., Haynes M. P., 1998, ApJ, 497, L1

Veilleux S., Osterbrock D. E., 1987, ApJs, 63, 295

Vincenzo F., Belfiore F., Maiolino R., Matteucci F., Ventura P., 2016, MNRAS, 458, 3466 
Voges E. S., Walterbos R. A. M., 2006, ApJ, 644, L29

Walterbos R. A. M., Braun R., 1994, ApJ, 431, 156

Wang J., Heckman T. M., Lehnert M. D., 1997, ApJ, 491, 114

Wilkinson D. M. et al., 2015, MNRAS, 449, 328

Wright S. A., Larkin J. E., Graham J. R., Ma C.-P., 2010, ApJ, 711, 1291

Wuyts E. et al., 2016, ApJ, 827, 74

Yan R., Blanton M. R., 2012, ApJ, 747, 61

Yan R., Newman J. A., Faber S. M., Konidaris N., Koo D., Davis M., 2006, ApJ, 648, 281

Yan R. et al., 2016a, AJ, 151, 8

Yan R. et al., 2016b, ApJ, 152, 197

Yates R. M., Kauffmann G., Guo Q., 2012, MNRAS, 422, 215

Yuan T.-T., Kewley L. J., Swinbank A. M., Richard J., Livermore R. C., 2011, ApJ, 732, L14
Zaritsky D., Kennicutt R. C., Jr, Huchra J. P., 1994, ApJ, 420, 87

Zhang K., Wang T., Dong X., Lu H., 2008, ApJ, 685, L109

Zurita A., Rozas M., Beckman J. E., 2000, A\&A, 363, 9

Zurita A., Beckman J. E., Rozas M., Ryder S., 2002, A\&A, 386, 801

\section{APPENDIX A: BEAM SMEARING EFFECT}

Most of the $\mathrm{H}_{\mathrm{II}}$ reigon is smaller than $1 \mathrm{kpc}$, and the size depends on the density of the gas (e.g. Hunt \& Hirashita 2009). Under MaNGA resolution, the mixing of $\mathrm{H}$ II region with DIG and other $\mathrm{H}$ II region is unavoidable. What is the effect of this mixing? We have seen in Section 3.1 that in one annulus, the line ratio versus surface brightness relation is tight. What would happen if we mix
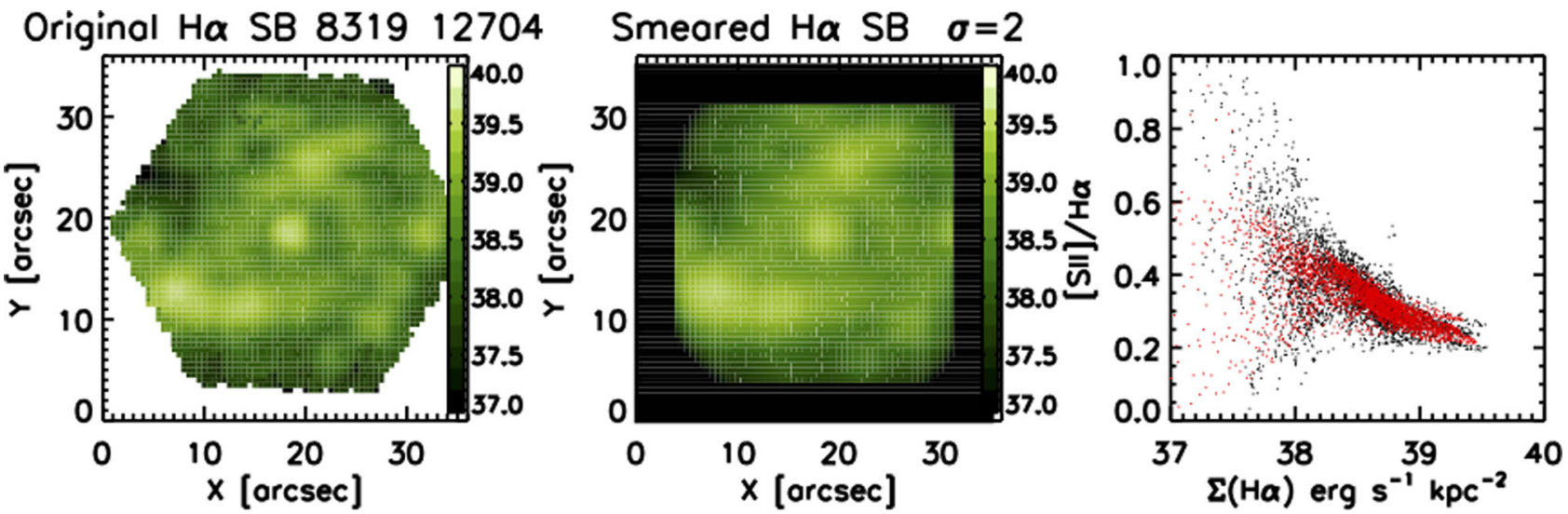

Original Ha SB 831912704

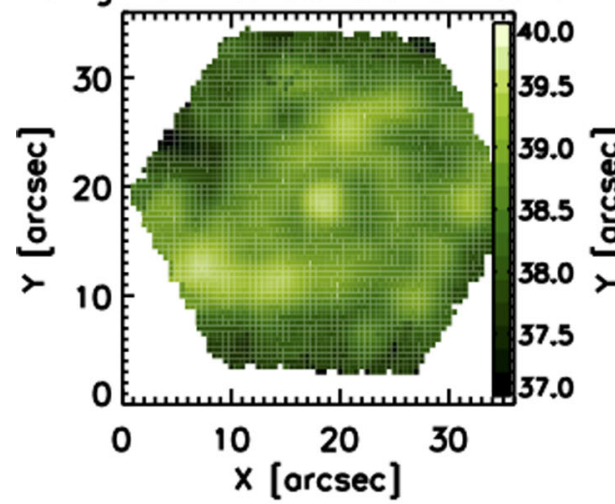

Smeared Ha SB $\quad \sigma=4$
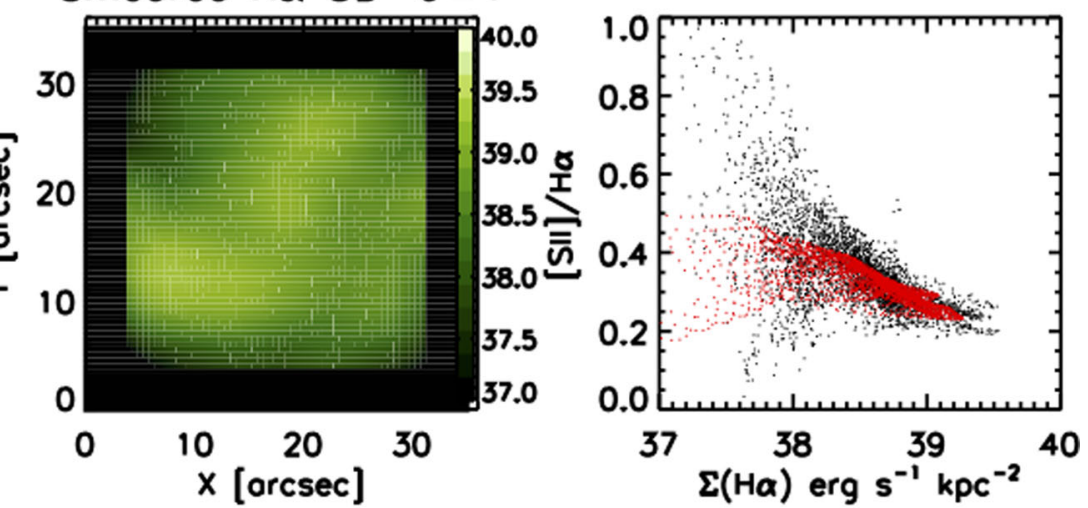

Original Ha SB 831912704

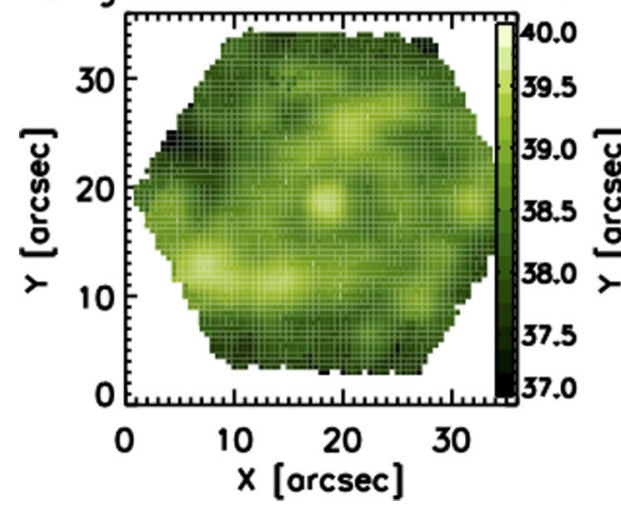

Smeared Ha SB $\sigma=7$
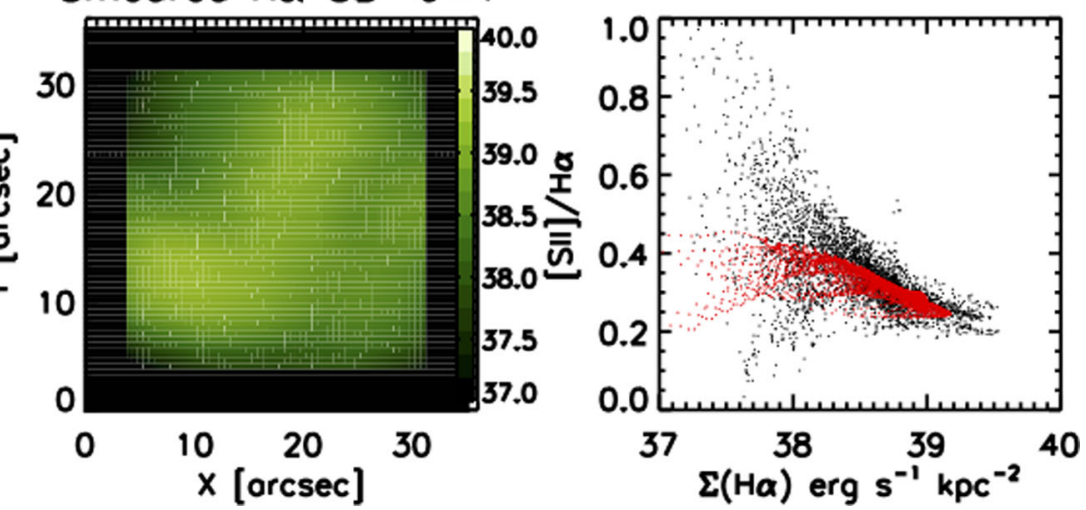

Figure A1. Left-hand panel: the original $\Sigma(\mathrm{H} \alpha)$ map, middle panel: the smeared $\Sigma(\mathrm{H} \alpha)$ map. Right panel: the comparison of original (black dots) and smeared (red dots) line ratio versus $\mathrm{H} \alpha$ surface brightness relation. The $\mathrm{H} \alpha$ flux map and [N II] flux map is convolved with a 2D Gaussian with $\sigma$ pixels listed in the middle panels of each row. 
two regions on the line ratio versus surface brightness relation? From an analytical calculation, the mixed point is still on the same relation. However, if we mix two or more $\mathrm{H}$ II regions together, what would the line ratio versus $\mathrm{H} \alpha$ surface brightness relation like? We show in Fig. A1 a simulation of observing our galaxies with poorer resolution. The left-hand panel is the original $\Sigma(\mathrm{H} \alpha)$ map, and the middle panel is the smeared $\Sigma(\mathrm{H} \alpha)$ map. In the right-hand panel, we show the comparison of original (black dots) and smeared (red dots) line ratio versus $\mathrm{H} \alpha$ surface brightness relation. The $\mathrm{H} \alpha$ flux map and [ $\mathrm{S}_{\text {II] }}$ flux map is convolved with a 2D Gaussian with $\sigma$ pixels listed in the middle panels of each row. We see several facts from this exercise.

(i) The beam smearing effect does not change the line ratio versus $\mathrm{SB}$ relation for one $\mathrm{H}$ II region, so the curve we derive using MaNGA data is likely to preserve when higher resolution data are obtained. In other word, this relation is intrinsic, reflecting the relationship between line ratio and surface brightness. Our resolution of $\sim 1 \mathrm{kpc}$ means that one $\mathrm{H} \alpha$ flux peak in our map is probably a mixture of several $\mathrm{H}$ II regions.

(ii) It is impossible to disentangle DIG from $\mathrm{H}$ II region from the curve alone.

(iii) The smearing makes the relation tighter and shorter.

(iv) When mixing two or more $\mathrm{H}_{\text {II }}$ regions together, their line ratio versus $\mathrm{SB}$ relations also merge to an intermediate one.

The smearing process is an averaging process. We are witnessing more and more of the average properties of the $\mathrm{H}$ II region and DIG of the galaxy as we go to poorer resolution. The overall smeared line ratio versus $\mathrm{H} \alpha$ surface brightness relation is tighter than before. So the tightness of the relation we see should be partly due to the beam smearing effect.

\footnotetext{
${ }^{1}$ Department of Physics and Astronomy, University of Kentucky, 505 Rose Street, Lexington, KY 40506, USA

${ }^{2}$ Kavli Institute for the Physics and Mathematics of the Universe (Kavli IPMU, WPI), Todai Institutes for Advanced Study, the University of Tokyo, Kashiwa 277-8583, Japan

${ }^{3}$ Department of Astronomy, University of Wisconsin-Madison, 475 N. Charter Street, Madison, WI, 53706, USA
}

${ }^{4}$ Department of Astronomy, New Mexico State University, Las Cruces, NM 88003, USA

${ }^{5}$ Cavendish Laboratory, University of Cambridge, 19 J. J. Thomson Avenue, Cambridge CB3 OHE, UK

${ }^{6}$ Kavli Institute for Cosmology, University of Cambridge, Madingley Road, Cambridge, UK

${ }^{7}$ Institute of Cosmology and Gravitation, University of Portsmouth, Dennis Sciama Building, Portsmouth PO1 3FX, UK

${ }^{8}$ Department of Astronomy, University of Texas at Austin, Austin, TX 78712, USA

${ }^{9}$ Max-Planck-Institut für Astrophysik, Karl-Schwarzschild-Str. 1, D-85748 Garching, Germany

${ }^{10}$ Instituto de Astronomía, Universidad Nacional Autonoma de Mexico, A.P. 70-264, 04510, Mexico, D.F., Mexico

${ }^{11}$ Apache Point Observatory and New Mexico State University, P.O. Box 59, Sunspot, NM 88349-0059, USA

${ }^{12}$ Sternberg Astronomical Institute, Moscow State University, Moscow, Russia

${ }^{13}$ Unidad de Astronomía, Universidad de Antofagasta, Avenida Angamos 601, Antofagasta 1270300, Chile

${ }^{14}$ PITT PACC, Department of Physics and Astronomy, University of Pittsburgh, Pittsburgh, PA 15260, USA

${ }^{15}$ Department of Physics and Astronomy, University of Utah, 115 S. 1400 E., Salt Lake City, UT 84112, USA

${ }^{16}$ Tsinghua Center of Astrophysics and Department of Physics, Tsinghua University, Beijing 100084, China

${ }^{17}$ Shanghai Astronomical Observatory, Nandan Road 80, Shanghai 200030, China

${ }^{18}$ Space Telescope Science Institute, 3700 San Martin Drive, Baltimore, MD 21218, USA

${ }^{19}$ Departamento de Física, Facultad de Ciencias, Universidad de La Serena, Cisternas 1200, La Serena, Chile

${ }^{20}$ Universidade Federal do Rio Grande do Sul, IF, CP 15051, Porto Alegre 91501-970, RS, Brazil

${ }^{21}$ Laboratório Interinstitucional de e-Astronomia - LIneA, Rua Gal. José Cristino 77, Rio de Janeiro, RJ - 20921-400, Brazil

This paper has been typeset from a $\mathrm{T}_{\mathrm{E}} \mathrm{X} / \mathrm{L} \mathrm{T} \mathrm{EX}$ file prepared by the author. 